\title{
Relocation Impacts of an H-Separations Coil and Tube Failure
}

by

A. Blanchard

Westinghouse Savannah River Company

Savannah River Site

Aiken, South Carolina 29808

J. M. Thompson

Westinghouse Safety Management Solutions

E. A. Thompson

Westinghouse Safety Management Solutions

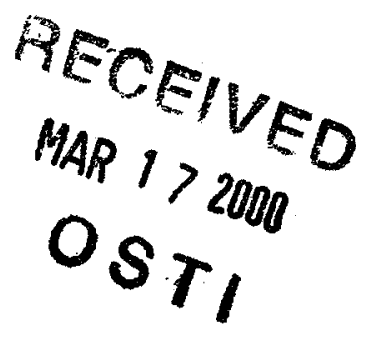

This paper was prepared in connection with work done under the above contract number with the U.S. Department of Energy. By acceptance of this paper, the publisher and/or recipient acknowledges the U.S. Government's right to retain a nonexclusive, royalty-free license in and to any copyright covering this paper, along with the right to reproduce and to authorize others to reproduce all or part of the copyrighted paper. 
Keywords: Relocation

Emergency Planning

Retention: Lifetime

\title{
Relocation Impacts of an H-Separations Coil and Tube Failure (U)
}

\author{
Elizabeth A. Thompson
}

Jay M. Thompson

June 1999

Classification

UNCLASSIFIED

DOES NOT CONTAIN

UNCLASSIFIED CONTROLLED

NUCLEAR INFORMATION

Westinghouse Savannah River Company Technical Services Division

Aiken, SC 29808
ADC \&

Reviewing

Official:
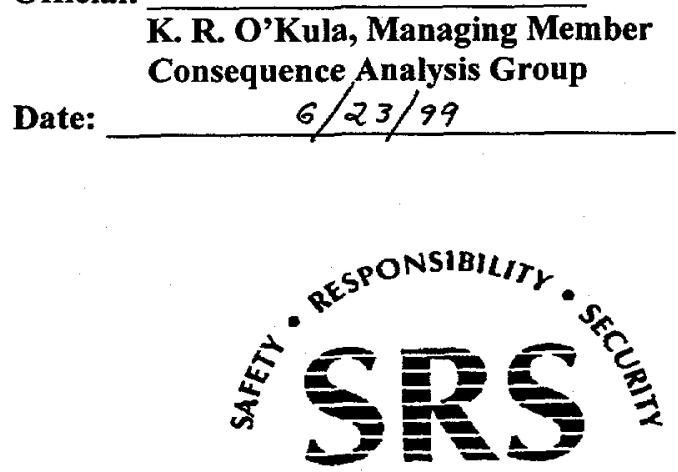

SAVANNAH RIVER SITE

PREPARED FOR THE U.S. DEPARTMENT OF ENERGY UNDER CONTRACT NO. DE-AC09-96SR18500 


\title{
Relocation Impacts of an H-Separations Coil and Tube Failure (U)
}

\author{
Elizabeth A. Thompson
}

Jay M. Thompson

June 1999

Westinghouse Savannah River Company Technical Services Division Aiken, SC 29808

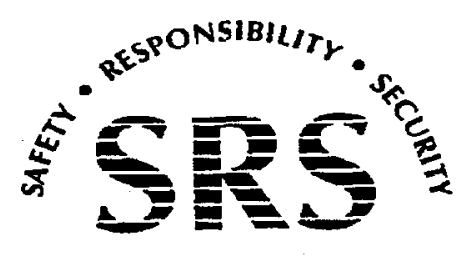


WSRC-TR-99-00104

Revision 1

\section{DISCLAIMER}

This report was prepared as an account of work sponsored by an agency of the United States Government. Neither the United States Government nor any agency thereof, nor any of their employees, makes any warranty, express or implied, or assumes any legal liability or responsibility for the accuracy, completeness, or usefulness of any information, apparatus, product or process disclosed, or represents that its use would not infringe privately owned rights. Reference herein to any specific commercial product, process or service by trade name, trademark, manufacturer, or otherwise does not necessarily constitute or imply its endorsement, recommendation, or favoring e) by the United States Government or any agency thereof. The views and opinions of authors If expressed herein do not necessarily state or reflect those of the United States Government or any p. agency thereof.

$\mathbf{R}$

This report has been reproduced directly from the best available copy.

$\mathbf{r}$

Available for sale to the public, in paper, from: U.S. Department of Commerce, National Technical Information Service, 5285 Port Royal Road, Springfield, VA 22161, phone: (800) 553-6847

fax: (703) 605-6900

email: orders@ntis.fedworld.gov

online ordering: http://www.ntis.gov/ordering.htm

A Available electronically at http://www.doe.gov/bridge Available for a processing fee to U.S. Department of Energy and its contractors, in paper, from:

A U.S. Department of Energy, Office of Scientifye, TN 37831-0062, phone: (865) 576-8401

C fax: (865) 576-5728

email: reports@adonis.osti.gov 


\section{DISCLAIMER}

Portions of this document may be illegible in electronic image products. Images are produced from the best available original document. 
WSRC-TR-99-00104

Revision 1

PROJECT:

DOCUMENT:

TITLE:

REVISION NUMBER:
NRA

WSRC-TR-99-00104

Relocation Impacts of an H-Separations Coil and Tube Failure (U)

AUTHOR/TECHNICAL REVIEWS:

E.A. Thompson

E. A. Thompson, Author

M.M. Thompson Author

DRay

D. R.Małx, Technical Reviewer

APPROVALS:

- 0.

C. E. Shogren, Managing Member, ESTG

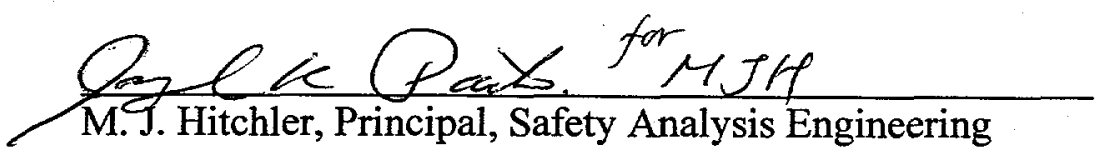

$\frac{6 / 14 / 99}{\text { Date }}$

$\frac{6 / 14 / 99}{\text { Date }}$

14fune 1999

Date

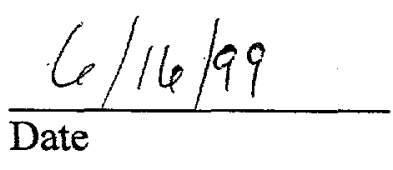

$\frac{6-16-99}{\text { Date }}$

i 


\begin{abstract}
The relocation impacts of an accidental release, scenario 1-RD-2.35, are evaluated for $\mathrm{H}$-Separations. The extent of the area potentially contaminated to a level that would result in doses exceeding the relocation protective action guide (PAG) is calculated. The maximum calculated distance downwind from the accident at which the relocation PAG is exceeded is also determined. The consequences of the release are evaluated using the HOTSPOT model and an EXCEL spreadsheet.
\end{abstract}




\section{CONTENTS}

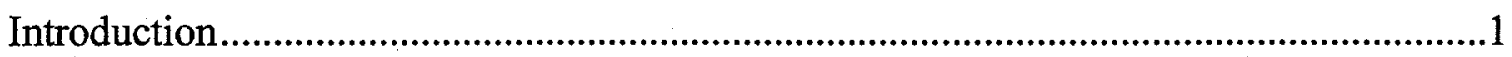

Approach

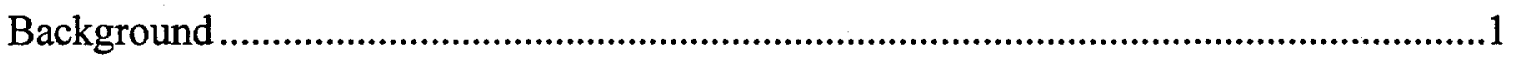

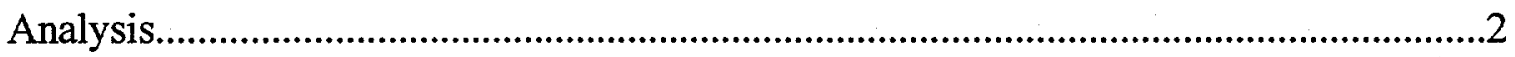

Source term

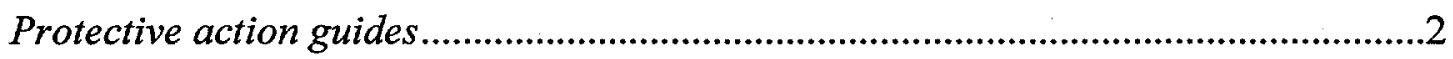

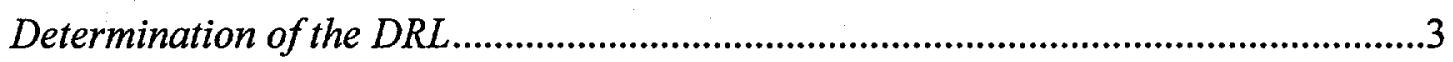

Determination of range and area of consequences ......................................................

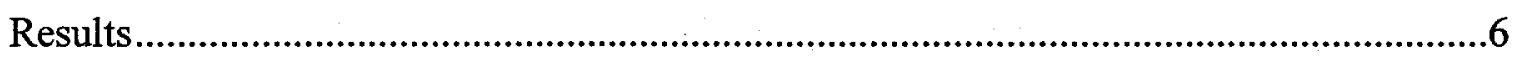

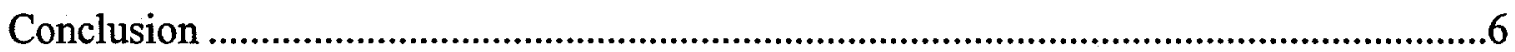

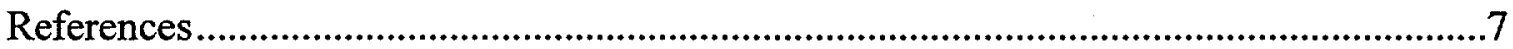

\section{TABLES}

Table 1. H-Separations airborne source term, 1-RD-2.35 …......................................... 2

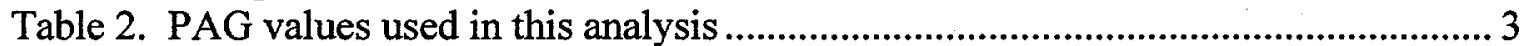

Table 3. Areal concentrations for a unit deposition............................................................ 4

Table 4. PAGs and DRLs..............................................................................................

Table 5. HOTSPOT Parameters used for the H-Separations Coil \& Tube release (1-RD-2.35) .....................................................................................................

Table 6. Ranges (in $\mathrm{km}$ ) until deposition less than relocation DRL, average meteorology, H-Separations, 1-RD-2.35 ........................................................................................ 6

Table 7. Areas (in $\mathrm{km}^{2}$ ) with deposition above relocation DRL, average meteorology, H-Separations, 1-RD-2.35

Table 8. Ranges (in $\mathrm{km}$ ) until deposition less than relocation DRL, 95\% adverse

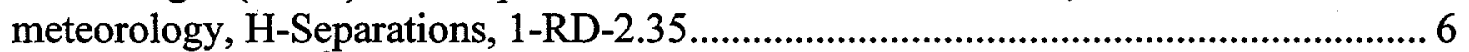

Table 9. Areas (in $\mathrm{km}^{2}$ ) with deposition above relocation DRL, 95\% adverse meteorology, H-Separations, 1-RD-2.35. 


\section{Introduction}

The relocation impacts of an accidental release, scenario 1-RD-2.35, are evaluated for $\mathrm{H}$-Separations. The sizes of the area requiring the protective action of relocation, as well as the maximum distances out to which protective action would be recommended, are calculated.

\section{Approach}

The methodology for determining the areas potentially requiring relocation is outlined below.

1. Determine the source terms (radionuclide-specific respirable and total airborne releases).

2. Determine the relocation Protective Action Guides (PAGs) for the radionuclides in the source terms in accordance with EPA (1992).

3. Calculate the doses (from inhalation and ground shine) associated with a unit deposition of (respirable) particulates. The inhalation source term is derived from the respirable airborne release. The ground shine source term is derived from the total (nonrespirable plus respirable) airborne release.

4. Sum the doses resulting from the unit deposition. Divide the relocation PAG by the sum of the doses to obtain the Derived Response Level (DRL) for relocation.

5. Run the dispersion/deposition model HOTSPOT (Homann 1994) to determine areas impacted.

\section{Background}

The area affected is considered to be that area in which the deposition due to an accidental release could cause the projected dose to an individual in that area to exceed the PAG. The Environmental Protection Agency (EPA) presents the rationale for its relocation PAG in its Manual of Protective Action Guides and Protective Actions for Nuclear Incidents (EPA 1992, section 4.2.1, p. 4-4 and Appendix E). The objectives of the relocation PAG are to keep the dose in any year after the first year following the accident from exceeding $500 \mathrm{mrem}(5 \mathrm{mSv})$, and to keep the cumulative dose over 50 years (including the first year) from exceeding 5,000 mrem (50 $\mathrm{mSv})$. For the reactor accidents addressed in EPA (1992), the PAG is 2,000 mrem (20 mSv) projected dose* ${ }^{*}$ Additionally, it specifies that at 50 times the numerical value of the PAG, relocation to limit beta exposure to skin would be warranted.

For fission product releases, relocation decisions are based on whole body irradiation (or effective dose equivalent); preferential irradiation of single tissues is not anticipated to be limiting. However, the radiological hazards associated with many radionuclides (such as plutonium and other actinides) differ significantly from those of the fission product mix considered in EPA (1992). Guidance from the ICRP gives dose limits to single tissues, based on

\footnotetext{
* The projected sum of effective dose equivalent from external gamma radiation and committed effective dose
} equivalent from inhalation of resuspended materials, from exposure or intake during the first year. 
non-stochastic effects that are a factor of ten greater than the effective dose limit based on stochastic effects (ICRP 1977). In general, the radiation associated with the decay of fission products is very penetrating and presents an external hazard (i.e., a person need only be exposed to the radioactive material to receive a dose; the material does not have to be ingested or inhaled) or a whole body irradiation hazard (material inside the body irradiates many tissues as it decays). The radiation associated with the decay of many actinides is not very penetrating and, therefore, only presents a significant radiological hazard when the material is taken into the body, either by inhalation or ingestion. Even then, specific organs are more limiting based on the radiosensitivity of the organ and the distribution of the radionuclides in the body. For the inhalation and ingestion pathways the dose to bone surfaces is more limiting than the effective dose for many actinides.

In order to facilitate comparisons between measured quantities and projected doses, it is often convenient to express a projected dose in terms of a measurable quantity. The value of a measurable quantity that corresponds to a dose value of interest (such as a PAG) is referred to as a derived response level (DRL). Spreadsheet calculations were performed to determine the relocation DRLs for the nuclide mix of interest. These DRLs were then used in conjunction with the HOTSPOT computer model to determine the size or extent of the area affected.

\section{Analysis}

\section{Source term}

For H-Separations, the postulated source term for the Coil \& Tube Release Scenario, 1-RD-2.35, is evaluated. Respirable releases are the same as total releases for each radionuclide since the respirable fraction (RF) is 1.0 (S-CLC-G-00004, Sheet 6, section 3.2). S-CLC-G-00004 contains the source term calculations used ultimately in the Interim Emergency Preparedness Hazards Assessment for H Area Separations, S-EHA-H-00004 (Burns and Clifton 1998). The source terms are listed in Table 1.

Table 1. H-Separations airborne source term, 1-RD-2.35

\begin{tabular}{cc}
\hline Nuclide & Airborne Release (Ci) \\
\hline Am-241 & $6.57 \mathrm{E}-01$ \\
Pu-238 & $1.62 \mathrm{E}+00$ \\
Pu-239 & $4.23 \mathrm{E}-03$ \\
Pu-240 & $2.43 \mathrm{E}-01$ \\
Pu-241 & $3.26 \mathrm{E}+01$ \\
Pu-242 & $1.54 \mathrm{E}-02$ \\
Total & $3.51 \mathrm{E}+01$ \\
\hline
\end{tabular}

Reference: Source_terms_reloc.xls, Worksheet "Relo_H", column C.

\section{Protective action guides}

For evaluating the size of the area affected by potential accidents, relocation PAGs were used. Consideration was given to both effective dose and dose to bone surfaces. Since dose limits to single tissues, based on non-stochastic effects, are a factor of ten greater than the effective dose limit based on stochastic effects (ICRP 1977); the limit used for single tissues (e.g., bone 
surfaces) was ten times that used for effective dose. These PAG values were used for evaluating the size of the area in which relocation may be warranted as a consequence of potential accidents.

For releases consisting primarily of long-lived radionuclides, the relocation PAG level of 2,000 mrem ( $20 \mathrm{mSv})$ in the first year may not, by itself, provide the intended level of long-term protection. The relocation PAG is intended to prevent doses exceeding $500 \mathrm{mrem}(5 \mathrm{mSv})$ in any year after the first year following the accident, or $5,000 \mathrm{mrem}(50 \mathrm{mSv})$ over the fifty years immediately following the accident. The relocation PAG values for projected doses that were considered for this work are presented in Table 2.

Table 2. PAG values used in this analysis

\begin{tabular}{ll}
\hline \multicolumn{1}{c}{ PAG } & \multicolumn{1}{c}{ Timeframe } \\
\hline $\begin{array}{ll}2,000 \text { mrem }(20 \mathrm{mSv}) \text { effective dose, or } 20,000 \mathrm{mrem}(200 \mathrm{mSv}) \\
\text { committed dose equivalent to a single tissue or organ }\end{array}$ & $\begin{array}{l}\text { the first year following an } \\
\text { accident }\end{array}$ \\
$500 \mathrm{mrem}(5 \mathrm{mSv})$ effective dose, or $5,000 \mathrm{mrem}(50 \mathrm{mSv})$ & $\begin{array}{l}\text { any year after the first year } \\
\text { following an accident }\end{array}$ \\
$\begin{array}{l}5,000 \mathrm{mrem}(50 \mathrm{mSv}) \text { effective dose, or } 50,000 \mathrm{mrem}(500 \mathrm{mSv}) \\
\text { committed dose equivalent to a single tissue or organ }\end{array}$ & $\begin{array}{l}\text { the } 50 \text { years (including the first } \\
\text { year) following an accident }\end{array}$ \\
\hline
\end{tabular}

The effective dose is the "projected sum of effective dose equivalent from external gamma radiation and committed effective dose equivalent from inhalation of resuspended materials, from exposure or intake during the" time period of interest (EPA 1992). Similarly, the dose to an individual organ should consider both the committed dose from any intake plus any external contribution to dose, if significant.

\section{Determination of the DRL}

The respirable fraction (RF) is equal to unity for the release considered here, so the respirable release is equal to the total release. The first step in determining the DRL is to assume a unit deposition $\left(1 \mu \mathrm{Ci} / \mathrm{m}^{2}\right)$ of the nuclide mix of interest, and that all radionuclides in the mix deposit similarly. In this case, the relative concentrations of the deposited material will be the same as that released.

The deposited activity can be apportioned to the individual nuclides based on the activity fraction (the ratio of the individual nuclide activity to the total activity) of the material released. That is: where

$$
d e p_{i}=\operatorname{dep}_{\text {Total }}\left(\frac{A_{i}}{A_{\text {Total }}}\right)
$$

$d e p_{i}=$ the deposition concentration of the $\mathrm{i}^{\text {th }}$ nuclide in $\mu \mathrm{Ci} / \mathrm{m}^{2}$

$\operatorname{dep}_{\text {Total }}=$ the total deposition concentration $\left(1 \mu \mathrm{Ci} / \mathrm{m}^{2}\right)$

$A_{i}=$ the activity of the $\mathrm{i}^{\text {th }}$ nuclide (Ci)

$A_{\text {Total }}=$ the total activity released $(\mathrm{Ci})$

The quantities of the individual radionuclides present for this unit deposition are given in Table 3. Attachment A presents applicable spreadsheet calculations. 
Table 3. Areal concentrations for a unit deposition

\begin{tabular}{cc}
\hline Nuclide & $\begin{array}{c}\text { Areal concentration }\left(\mu \mathrm{Ci} \mathrm{m}^{-2}\right) \text { per } \\
1 \mu \mathrm{Ci} \mathrm{m}{ }^{-2} \text { deposition of mix }\end{array}$ \\
\hline $\mathrm{Am}-241$ & $1.87 \mathrm{E}-02$ \\
$\mathrm{Pu}-238$ & $4.61 \mathrm{E}-02$ \\
$\mathrm{Pu}-239$ & $1.20 \mathrm{E}-04$ \\
$\mathrm{Pu}-240$ & $6.92 \mathrm{E}-03$ \\
$\mathrm{Pu}-241$ & $9.28 \mathrm{E}-01$ \\
$\mathrm{Pu}-242$ & $4.38 \mathrm{E}-04$ \\
\hline
\end{tabular}

Reference: Source_terms_reloc.xls, Worksheet "Relo_H", column D

Inhalation dose conversion factors (DCFs) are from Federal Guidance Report 11 (EPA 1988). The lung clearance class for each nuclide was chosen to maximize the effective dose. For most nuclides this also maximized the bone surface DCF. External exposure DCFs are from DOE/EH-0070 (1988). To simplify calculations, and due to the anticipated small contribution of the external dose relative to the internal doses, the external contribution to the bone surface dose was approximated by the external contribution to the effective dose. For this nuclide mix and exposure scenario, this approximation did not introduce any appreciable error.

The amount of deposition for each nuclide determined above is used to calculate the dose contributed by that nuclide for both the external gamma exposure and inhalation of resuspended material pathways for both effective and organ doses. These internal and external components are then summed over the nuclides present and combined to give the effective dose and the organ dose equivalent due to a unit deposition of material. These calculations consider weathering and are performed for three time periods. Specifically, the cases considered were those indicated in Table 2 above with the dose in "any year after the first year following an accident" being represented by the dose in the second year following an accident. Weathering is modeled using the method presented in EPA (1992) where the weathering factor $(W F)$ is defined as

where

$$
W F=\frac{0.63}{\lambda_{1}+\lambda_{2}}\left(1-e^{-\left(\lambda_{1}+\lambda_{2}\right) t}\right)+\frac{0.37}{\lambda_{1}+\lambda_{3}}\left(1-e^{-\left(\lambda_{1}+\lambda_{3}\right) t}\right)
$$

$\mathrm{t}=$ time during which radionuclides are inhaled (or exposure occurs),

$\lambda_{1}=$ radioactive decay constant,

$\lambda_{2}=$ assumed weathering decay constant for 63 percent of the deposited activity, taken as 1.13 per year, and

$\lambda_{3}=$ assumed weathering decay constant for 37 percent of the deposited activity, taken as $7.48 \mathrm{E}-3$ per year.

This weathering factor is used as the time period over which exposure occurs.

For a deposition of $1 \mu \mathrm{Ci} \mathrm{m}^{-2}$ of the 1-RD-2.35 mix and a fifty-year exposure period, the inhalation dose (CEDE) is 4,950 mrem, the committed dose to bone surfaces (BS dose) is 89,500 $\mathrm{mrem}$, and the external exposure is $0.9 \mathrm{mrem}$. Adding the external and internal doses gives $4,950 \mathrm{mrem}$ effective dose and $89,500 \mathrm{mrem}$ to bone surfaces for a deposition of $1 \mu \mathrm{Ci} \mathrm{m}{ }^{-2}$ of activity. 
The DRLs may now be determined by dividing the PAGs by their corresponding dose per unit deposition. The DRLs associated with the effective and bone surface doses are much more limiting than those associated with the skin dose and are presented in Table 4. "Bone-surface dose" is the CDE to the bone surfaces. The DRLs calculated using the dose due to fifty years of exposure are the most limiting. Therefore, the DRLs presented for further analysis are those associated with effective and bone surface doses for fifty years of exposure. Details of the spreadsheet calculations are in Attachment A.

Table 4. PAGs and DRLs

\begin{tabular}{cccc}
\hline $\begin{array}{c}\text { PAG } \\
(\mathrm{mrem})\end{array}$ & Description & $\begin{array}{c}\mathrm{DRL} \\
\left(\mu \mathrm{Ci} \mathrm{m}^{-2}\right)\end{array}$ & DRL Formula \\
\hline 2000 & Effective dose, first year, no weathering & 5.1 & DRL = PAG/(CEDE+External) \\
20000 & Bone-surface dose, first year, no weathering & 2.8 & DRL = PAG/(BS Dose + External) \\
2000 & Effective dose, first year, with weathering & 6.9 & DRL = PAG/(CEDE+External) \\
20000 & Bone-surface dose, first year, with weathering & 3.8 & DRL = PAG/(BS Dose + External) \\
500 & Effective dose, second year, with weathering & 2.7 & DRL = PAG/(CEDE+External) \\
5000 & Bone-surface dose, second year, with & 1.5 & DRL = PAG/(BS Dose + External) \\
& weathering & & \\
5000 & Effective dose, fifty years, with weathering & 1.0 & DRL = PAG/(CEDE+External) \\
50000 & Bone-surface dose, fifty years, with & 0.56 & DRL $=$ PAG/(BS Dose + External) \\
& weathering & & \\
\hline
\end{tabular}

Reference: Source_terms_reloc.xls_50yr, Worksheet "Relo_H DRLs Summary".

\section{Determination of range and area of consequences}

The HOTSPOT computer code was selected to evaluate the consequences of particulate releases (WSRC-TR-98-00392). In the following calculations, a source term equal to the total activity $(35.1 \mathrm{Ci})$ is used, and the range and area affected for different meteorological parameters and DRLs are found. Pasquill-Briggs dispersion parameters are used.

HOTSPOT Parameters used for the H-Area Coil and Tube release are listed in Table 5.

Table 5. HOTSPOT Parameters used for the H-Separations Coil \& Tube release (1-RD-2.35)

\begin{tabular}{ccc}
\hline Parameter & Average Meteorology & $95 \%$ Adverse Meteorology \\
\hline Release height & Ground & Ground \\
Wind speed & $2.5 \mathrm{~m} / \mathrm{s}$ & $1.7 \mathrm{~m} / \mathrm{s}$ \\
Surface roughness & $100 \mathrm{~cm}$ & $100 \mathrm{~cm}$ \\
Stability class & $\mathrm{C}$ & $\mathrm{E}$ \\
Deposition velocity & $0.1 / 1 / 10 \mathrm{~cm} / \mathrm{sec}$ & $0.1 / 1 / 10 \mathrm{~cm} / \mathrm{sec}$ \\
Release duration & $120 \mathrm{~min}$ & $120 \mathrm{~min}$ \\
Inversion layer & $500 \mathrm{~m}$ & $200 \mathrm{~m}$ \\
\hline
\end{tabular}

Meteorological data are consistent with the HSEP IEPHA, Rev. 1 (S-EHA-H-00004 Interim Emergency Preparedness Hazards Assessment for H Area Separations, Appendix B, Calculation 
5, Sheet 1), which references Consequence Assessment for Emergency Preparedness Hazards Assessments, TP-95-002, Revision 3, January 21, 1997.

\section{Results}

Tables 6 through 9 present results obtained for an H-Separations Coil and Tube Failure accident using the HOTSPOT computer code. The plots from which these data were obtained are included in Attachments B through G. For the average meteorology case, Tables 6 and 7 present the ranges and areas, respectively. Tables 8 and 9 contain results for runs made using adverse meteorological data.

Table 6. Ranges (in $\mathrm{km}$ ) until deposition less than relocation DRL, average meteorology, H-Separations, 1-RD-2.35

\begin{tabular}{ccccc}
\hline Basis for DRL & DRL & \multicolumn{3}{c}{ Deposition Velocity } \\
\cline { 3 - 5 } & $(\mu \mathrm{Ci} \mathrm{m})$ & $0.1 \mathrm{~cm} \mathrm{~s}^{-1}$ & $1 \mathrm{~cm} \mathrm{~s}^{-1}$ & $10 \mathrm{~cm} \mathrm{~s}^{-1}$ \\
\hline Effective dose & $1.0 \mathrm{E}+00$ & 0.25 & 0.82 & 1.8 \\
Bone Surface Dose & $5.6 \mathrm{E}-01$ & 0.34 & 1.1 & 3.5 \\
\hline
\end{tabular}

Table 7. Areas (in $\mathrm{km}^{2}$ ) with deposition above relocation DRL, average meteorology, H-Separations, 1-RD-2.35

\begin{tabular}{ccccc}
\hline Basis for DRL & DRL & \multicolumn{3}{c}{ Deposition Velocity } \\
\cline { 3 - 5 } & $\left(\mu \mathrm{Ci} \mathrm{m}^{-2}\right)$ & $0.1 \mathrm{~cm} \mathrm{~s}^{-1}$ & $1 \mathrm{~cm} \mathrm{~s}^{-1}$ & $10 \mathrm{~cm} \mathrm{~s}^{-1}$ \\
\hline Effective dose & $1.0 \mathrm{E}+00$ & $1.7 \mathrm{E}-02$ & $1.7 \mathrm{E}-01$ & $8.2 \mathrm{E}-01$ \\
Bone Surface Dose & $5.6 \mathrm{E}-01$ & $3.0 \mathrm{E}-02$ & $3.0 \mathrm{E}-01$ & $1.9 \mathrm{E}-00$ \\
\hline
\end{tabular}

Table 8. Ranges (in $\mathrm{km}$ ) until deposition less than relocation DRL, 95\% adverse meteorology, H-Separations, 1-RD-2.35

\begin{tabular}{ccccc}
\hline Basis for DRL & DRL & \multicolumn{3}{c}{ Deposition Velocity } \\
\cline { 3 - 5 } & $\left.(\mu \mathrm{Ci} \mathrm{m})^{-2}\right)$ & $0.1 \mathrm{~cm} \mathrm{~s}^{-1}$ & $1 \mathrm{~cm} \mathrm{~s}^{-1}$ & $10 \mathrm{~cm} \mathrm{~s}^{-1}$ \\
\hline Effective dose & $1.0 \mathrm{E}+00$ & 0.9 & 3.5 & 2.4 \\
Bone Surface Dose & $5.6 \mathrm{E}-01$ & 1.3 & 5.5 & 3.3 \\
\hline
\end{tabular}

Table 9. Areas (in $\mathrm{km}^{2}$ ) with deposition above relocation DRL, 95\% adverse meteorology, H-Separations, 1-RD-2.35

\begin{tabular}{ccccc}
\hline Basis for DRL & $\mathrm{DRL}$ & \multicolumn{3}{c}{ Deposition Velocity } \\
\cline { 3 - 5 } & $\left.(\mu \mathrm{Ci} \mathrm{m})^{-2}\right)$ & $0.1 \mathrm{~cm} \mathrm{~s}^{-1}$ & $1 \mathrm{~cm} \mathrm{~s}^{-1}$ & $10 \mathrm{~cm} \mathrm{~s}^{-1}$ \\
\hline Effective dose & $1.0 \mathrm{E}+00$ & $9.1 \mathrm{E}-02$ & $1.1 \mathrm{E}+00$ & $6.4 \mathrm{E}-01$ \\
Bone Surface Dose & $5.6 \mathrm{E}-01$ & $1.8 \mathrm{E}-01$ & $2.4 \mathrm{E}+00$ & $1.1 \mathrm{E}+00$ \\
\hline
\end{tabular}

\section{Conclusion}

Since the site boundary is over $11 \mathrm{~km}$ from $\mathrm{H}$-area, no relocation protective actions are likely to be recommended for this scenario. 


\section{References}

Burns, R. D. and Clifton, L. L. Interim Emergency Preparedness Hazards Assessment for $H$ Area Separations, Aiken, SC: Westinghouse Savannah River Company; S-EHA-H-00004, Rev. 1; June 1998.

Department of Energy. External Dose-Rate Conversion Factors for Calculation of Dose to the Public. Washington, DC: DOE; DOE/EH-0070, 1988.

Eisele, D. P. Risks of $F \& H$ Cooling Tower Airborne Release (U), Aiken, SC: Westinghouse Savannah River Company; S-CLC-G-00004, Rev. 0; May 1994.

Environmental Protection Agency. Limiting Values of Radionuclide Intake and Air Concentration and Dose Conversion Factors for Inhalation, Submersion, and Ingestion. Washington, DC: EPA; Federal Guidance Report No. 11, EPA 520/1-88-020, 1988.

Environmental Protection Agency. Manual of Protective Action Guides and Protective Actions for Nuclear Incidents. Washington, DC: EPA; EPA 400-R-92-001, 1992.

Hadlock, D. J., Consequence Assessment for Emergency Preparedness Hazards Assessments, Aiken, SC: Westinghouse Savannah River Company; TP-95-002, Rev. 3, 1/20/97.

Homann, Steven G., HOTSPOT Health Physics Codes for the PC, UCRL-MA-106315, Lawrence Livermore National Laboratory, University of California, Livermore, CA, March 1994.

International Commission on Radiological Protection. Recommendations of the International Commission on Radiological Protection. Oxford: Pergamon Press; ICRP Publication 26; Ann. ICRP 1(3); 1977.

Thompson, J. M. Selection of Models for Ingestion Pathway and Relocation (U), Aiken, SC; Westinghouse Savannah River Company; WSRC-TR-98-00392, Rev. 2; February, 1999. 


\section{Attachments}

Attachment A

Spreadsheet calculations

Attachment B

HOTSPOT results - average meteorology, $0.1 \mathrm{~cm} \mathrm{~s}^{-1}$

Attachment C

HOTSPOT results - average meteorology, $1.0 \mathrm{~cm} \mathrm{~s}^{-1}$

Attachment D

HOTSPOT results - average meteorology, $10 \mathrm{~cm} \mathrm{~s}^{-1}$

Attachment $\mathrm{E}$

HOTSPOT results - adverse meteorology, $0.1 \mathrm{~cm} \mathrm{~s}^{-1}$

Attachment $\mathrm{F}$

HOTSPOT results - adverse meteorology, $1.0 \mathrm{~cm} \mathrm{~s}^{-1}$

Attachment $G$

HOTSPOT results - adverse meteorology, $10 \mathrm{~cm} \mathrm{~s}^{-1}$ 


\section{Attachment A}

Spreadsheet calculations 


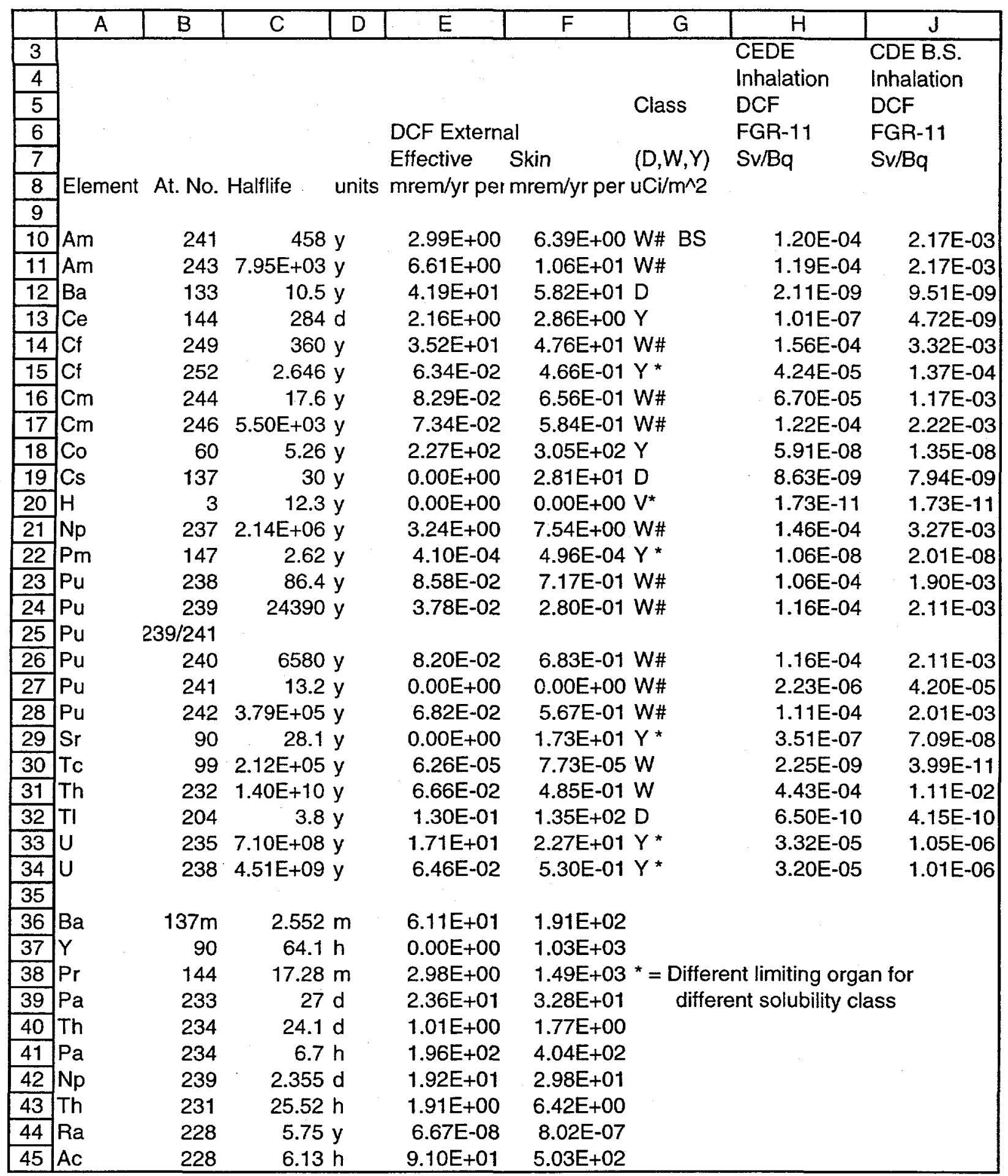




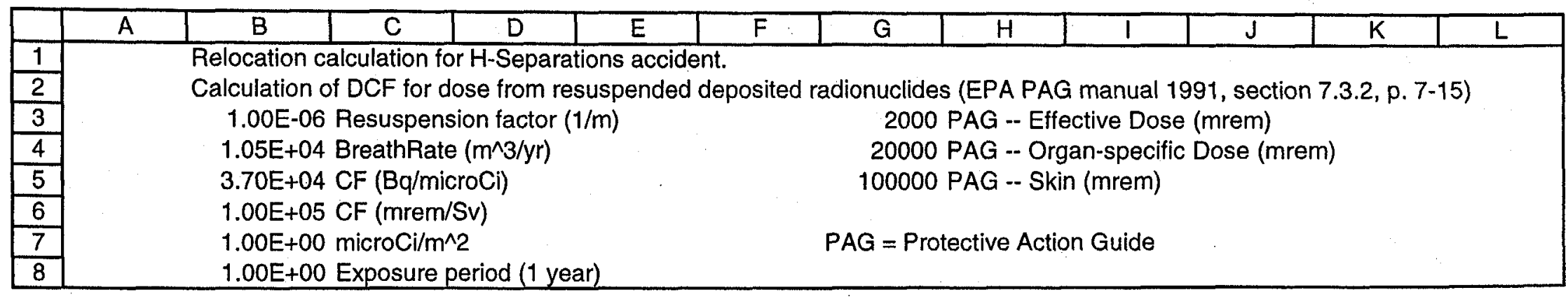




\begin{tabular}{|c|c|c|c|c|c|c|c|c|c|c|c|c|}
\hline & $\mathrm{B}$ & $\mathrm{C}$ & $\mathrm{D}$ & $E$ & $F$ & $\mathrm{G}$ & $\mathrm{H}$ & 1 & $\mathrm{~J}$ & $\mathrm{P}$ & $Q$ & $R$ \\
\hline 9 & Nuclide & $\begin{array}{l}\text { Release } \\
\text { (Ci) }\end{array}$ & $\begin{array}{l}\text { Activity } \\
\text { fraction }\end{array}$ & & $\begin{array}{l}\text { CEDE } \\
\text { (mrem per } \\
\mathrm{uCl} / \mathrm{m}^{\wedge} 2 \text { ) }\end{array}$ & $\begin{array}{l}\text { External } \\
\text { EDE } \\
\text { (mrem per } \\
\mathrm{uCi} / \mathrm{m}^{\wedge} 2 \text { ) }\end{array}$ & & $\begin{array}{l}\text { Skin Dose } \\
\text { (mrem per } \\
\left.\mathrm{uCi} / \mathrm{m}^{\wedge} 2\right)\end{array}$ & $\begin{array}{l}\text { BS Dose } \\
\text { (mrem per } \\
\mathrm{uCl} / \mathrm{m}^{\wedge} 2 \text { ) }\end{array}$ & & & \\
\hline 10 & Am-241 & 6.57E-01 & 1.87E-02 & & $8.72 E+01$ & 5.59E-02 & & 1.19E-01 & $1.58 \mathrm{E}+03$ & & & \\
\hline 11 & $A m-243$ & $0.00 E+00$ & $0.00 E+00$ & & $0.00 E+00$ & $0.00 \mathrm{E}+00$ & & $0.00 \mathrm{E}+00$ & $0.00 E+00$ & & & \\
\hline 12 & $\mathrm{Ba}-133$ & $0.00 E+00$ & $0.00 \mathrm{E}+00$ & & $0.00 E+00$ & $0.00 \mathrm{E}+00$ & & $0.00 \mathrm{E}+00$ & $0.00 E+00$ & & & \\
\hline 13 & $\mathrm{Ce}-144$ & $0.00 E+00$ & $0.00 \mathrm{E}+00$ & & $0.00 E+00$ & $0.00 E+00$ & & $0.00 \mathrm{E}+00$ & $0.00 \mathrm{E}+00$ & & & \\
\hline 14 & Cf-249 & $0.00 \mathrm{E}+00$ & $0.00 E+00$ & & $0.00 \mathrm{E}+00$ & $0.00 \mathrm{E}+00$ & & $0.00 \mathrm{E}+00$ & $0.00 \mathrm{E}+00$ & & & \\
\hline 15 & Cf-252 & $0.00 \mathrm{E}+00$ & $0.00 E+00$ & & $0.00 E+00$ & $0.00 E+00$ & & $0.00 E+00$ & $0.00 \mathrm{E}+00$ & & & \\
\hline 16 & $\mathrm{Cm}-244$ & $0.00 E+00$ & $0.00 E+00$ & & $0.00 E+00$ & $0.00 \mathrm{E}+00$ & & $0.00 E+00$ & $0.00 E+00$ & & & \\
\hline 17 & $\mathrm{Cm}-246$ & $0.00 E+00$ & $0.00 \mathrm{E}+00$ & & $0.00 E+00$ & $0.00 E+00$ & & $0.00 E+00$ & $0.00 \mathrm{E}+00$ & & & \\
\hline 18 & Co-60 & $0.00 E+00$ & $0.00 E+00$ & & $0.00 E+00$ & $0.00 E+00$ & & $0.00 E+00$ & $0.00 \mathrm{E}+00$ & & & \\
\hline 19 & Cs-137 & $0.00 E+00$ & $0.00 E+00$ & & $0.00 E+00$ & $0.00 E+00$ & & $0.00 E+00$ & $0.00 E+00$ & & & \\
\hline 20 & $\mathrm{H}-3$ & $0.00 \mathrm{E}+00$ & $0.00 E+00$ & & $0.00 E+00$ & $0.00 E+00$ & & $0.00 E+00$ & $0.00 E+00$ & & & \\
\hline 21 & Np-237 & $0.00 E+00$ & $0.00 E+00$ & & $0.00 E+00$ & $0.00 E+00$ & & $0.00 E+00$ & $0.00 E+00$ & & & \\
\hline 22 & $\mathrm{Pm}-147$ & $0.00 E+00$ & $0.00 \mathrm{E}+00$ & & $0.00 E+00$ & $0.00 \mathrm{E}+00$ & & $0.00 \mathrm{E}+00$ & $0.00 E+00$ & & & \\
\hline 23 & Pu-238 & $1.62 \mathrm{E}+00$ & 4.61E-02 & & $1.90 \mathrm{E}+02$ & $3.96 \mathrm{E}-03$ & & $3.31 E-02$ & $3.40 \mathrm{E}+03$ & & & \\
\hline 24 & Pu-239 & 4.23E-03 & $1.20 E-04$ & & 5.42E-01 & 4.55E-06 & & 3.37E-05 & $9.87 E+00$ & & & \\
\hline 25 & Pu-239/241 & $0.00 \mathrm{E}+00$ & $0.00 \mathrm{E}+00$ & & $0.00 \mathrm{E}+00$ & $0.00 E+00$ & & $0.00 \mathrm{E}+00$ & $0.00 \mathrm{E}+00$ & & & \\
\hline 26 & Pu-240 & 2.43E-01 & 6.92E-03 & & $3.12 E+01$ & 5.67E-04 & & 4.72E-03 & $5.67 \mathrm{E}+02$ & & & \\
\hline 27 & Pu-241 & $3.26 \mathrm{E}+01$ & $9.28 E-01$ & & $8.04 E+01$ & $0.00 E+00$ & & $0.00 E+00$ & $1.51 E+03$ & & & \\
\hline 28 & Pu-242 & $1.54 E-02$ & 4.38E-04 & & $1.89 E+00$ & 2.99E-05 & & 2.48E-04 & $3.42 E+01$ & & & \\
\hline 29 & Sr-90 & $0.00 E+00$ & $0.00 \mathrm{E}+00$ & & $0.00 E+00$ & $0.00 \mathrm{E}+00$ & & $0.00 \mathrm{E}+00$ & $0.00 \mathrm{E}+00$ & & & \\
\hline 30 & Tc-99 & $0.00 \mathrm{E}+00$ & $0.00 E+00$ & & $0.00 E+00$ & $0.00 E+00$ & & $0.00 E+00$ & $0.00 E+00$ & & & \\
\hline 31 & Th-232 & $0.00 E+00$ & $0.00 E+00$ & & $0.00 E+00$ & $0.00 \mathrm{E}+00$ & & $0.00 \mathrm{E}+00$ & $0.00 \mathrm{E}+00$ & & & \\
\hline 32 & TI-204 & $0.00 E+00$ & $0.00 E+00$ & & $0.00 E+00$ & $0.00 E+00$ & & $0.00 \mathrm{E}+00$ & $0.00 \mathrm{E}+00$ & & & \\
\hline 33 & U-235 & $0.00 E+00$ & $0.00 \mathrm{E}+00$ & & $0.00 E+00$ & $0.00 E+00$ & & $0.00 E+00$ & $0.00 \mathrm{E}+00$ & & & \\
\hline 34 & U-238 & $0.00 E+00$ & $0.00 E+00$ & & $0.00 E+00$ & $0.00 E+00$ & & $0.00 \mathrm{E}+00$ & $0.00 E+00$ & & & \\
\hline 35 & & & & & & & & & & & & \\
\hline 36 & $\mathrm{Ba}-137 \mathrm{~m}$ & $0.00 \mathrm{E}+00$ & $0.00 \mathrm{E}+00$ & & & & & & & & & \\
\hline 37 & $Y-90$ & $0.00 \mathrm{E}+00$ & $0.00 E+00$ & & & & & & & & & \\
\hline 38 & & & & & & & & & & & & \\
\hline 39 & Total & $3.51 E+01$ & $1.00 E+00$ & & $3.91 E+02$ & $6.05 \mathrm{E}-02$ & & $1.58 \mathrm{E}-01$ & $7.10 E+03$ & & & \\
\hline
\end{tabular}




\begin{tabular}{|c|c|c|c|c|}
\hline & B & $\mathrm{C}$ & $\bar{D}$ & $\bar{F}$ \\
\hline 9 & Nuclide & Release (Ci) & Activity fraction & CEDE (mrem per $\left.\mathrm{uCl} / \mathrm{m}^{\wedge} 2\right)$ \\
\hline 10 & Am-241 & $=$ Relocation!Y10 & $=\mathrm{C} 10 / \$ \mathrm{C} \$ 39$ & $=\$ B \$ 7^{*} \$ B \$ 3^{*} \$ B \$ 4^{*} \$ B \$ 5^{*} \$ B \$ 6^{*} \$ B \$ 8^{*} D 10^{*}$ Relocation! $H 10$ \\
\hline 11 & $A m-243$ & $=$ Relocation!Y11 & $=C 11 / \$ C \$ 39$ & $=\$ B \$ 7^{*} \$ B \$ 3^{*} \$ B \$ 4^{*} \$ B \$ 5^{*} \$ B \$ 6^{*} \$ B \$ 8^{*} D 11^{*}$ Relocation!H11 \\
\hline 12 & Ba-133 & $=$ Relocation!Y12 & $=C 12 / \$ C \$ 39$ & $=\$ B \$ 7^{*} \$ B \$ 3^{*} \$ B \$ 4^{*} \$ B \$ 5^{*} \$ B \$ 6^{*} \$ B \$ 8^{*} D 12^{*}$ Relocation!H12 \\
\hline 13 & $\mathrm{Ce}-144$ & $=$ Relocation!Y13 & $=\mathrm{C} 13 / \$ \mathrm{C} \$ 39$ & $=\$ B \$ 7^{*} \$ B \$ 3^{*} \$ B \$ 4^{*} \$ B \$ 5^{*} \$ B \$ 6^{*} \$ B \$ 8^{*} D 13^{*}$ Relocation!H13 \\
\hline 14 & Cf-249 & $=$ Relocation!Y14 & $=C 14 / \$ C \$ 39$ & $=\$ B \$ 7^{*} \$ B \$ 3^{*} \$ B \$ 4^{*} \$ B \$ 5^{*} \$ B \$ 6^{*} \$ B \$ 8^{*} D 14^{*}$ Relocation!H14 \\
\hline 15 & Cf-252 & $=$ Relocation!Y15 & $=C 15 / \$ C \$ 39$ & $=\$ B \$ 7^{*} \$ B \$ 3^{*} \$ B \$ 4^{*} \$ B \$ 5^{*} \$ B \$ 6^{*} \$ B \$ 8^{*} D 15^{*}$ Relocation!H15 \\
\hline 16 & $\mathrm{Cm}-244$ & $=$ Relocation!Y16 & $=C 16 / \$ C \$ 39$ & $=\$ B \$ 7^{*} \$ B \$ 3^{*} \$ B \$ 4^{*} \$ B \$ 5^{*} \$ B \$ 6^{*} \$ B \$ 8^{*} D 16^{*}$ Relocation!H16 \\
\hline 17 & $\mathrm{Cm}-246$ & $=$ Relocation!Y17 & $=C 17 / \$ C \$ 39$ & $=\$ B \$ 7^{*} \$ B \$ 3^{*} \$ B \$ 4^{*} \$ B \$ 5^{*} \$ B \$ 6^{*} \$ B \$ 8^{*} D 17^{*}$ Relocation! $\mathrm{H} 17$ \\
\hline 18 & Co-60 & $=$ Relocation!Y18 & $=\mathrm{C} 18 / \$ \mathrm{C} \$ 39$ & $=\$ B \$ 7^{*} \$ B \$ 3^{*} \$ B \$ 4^{*} \$ B \$ 5^{*} \$ B \$ 6^{*} \$ B \$ 8^{*} D 18^{*}$ Relocation!H18 \\
\hline 19 & Cs-137 & $=$ Relocation!Y19 & $=C 19 / \$ C \$ 39$ & $=\$ B \$ 7^{*} \$ B \$ 3^{*} \$ B \$ 4^{*} \$ B \$ 5^{*} \$ B \$ 6^{*} \$ B \$ 8^{*} D 19^{*}$ Relocation!H19 \\
\hline 20 & $\mathrm{H}-3$ & $=$ Relocation!Y20 & $=\mathrm{C} 20 / \$ \mathrm{C} \$ 39$ & $=\$ B \$ 7^{*} \$ B \$ 3^{*} \$ B \$ 4^{*} \$ B \$ 5^{*} \$ B \$ 6^{*} \$ B \$ 8^{*} D 20^{*}$ Relocation!H2O \\
\hline 21 & $\mathrm{~Np}-237$ & $=$ Relocation!Y21 & $=\mathrm{C} 21 / \$ \mathrm{C} \$ 39$ & $=\$ B \$ 7^{*} \$ B \$ 3^{*} \$ B \$ 4^{*} \$ B \$ 5^{*} \$ B \$ 6^{*} \$ B \$ 8^{*} D 21^{*}$ Relocation! $H 21$ \\
\hline 22 & $P m-147$ & $=$ Relocation!Y22 & $=\mathrm{C} 22 / \$ \mathrm{C} \$ 39$ & $=\$ B \$ 7^{*} \$ B \$ 3^{*} \$ B \$ 4^{*} \$ B \$ 5^{*} \$ B \$ 6^{*} \$ B \$ 8^{*} D 22^{*}$ Relocation!H 22 \\
\hline 23 & Pu-238 & $=$ Relocation!Y23 & $=\mathrm{C} 23 / \$ C \$ 39$ & $=\$ B \$ 7^{*} \$ B \$ 3^{*} \$ B \$ 4^{*} \$ B \$ 5^{*} \$ B \$ 6^{*} \$ B \$ 8^{*} D 23^{*}$ Relocation! H23 \\
\hline 24 & Pu-239 & $=$ Relocation!Y24 & $=\mathrm{C} 24 / \$ \mathrm{C} \$ 39$ & $=\$ B \$ 7^{*} \$ B \$ 3^{*} \$ B \$ 4^{*} \$ B \$ 5^{*} \$ B \$ 6^{*} \$ B \$ 8^{*} D 24^{*}$ Relocation! $\mathrm{H} 24$ \\
\hline 25 & Pu-239/241 & $=$ Relocation!Y25 & $=\mathrm{C} 25 / \$ \mathrm{C} \$ 39$ & $=\$ B \$ 7^{*} \$ B \$ 3^{*} \$ B \$ 4^{*} \$ B \$ 5^{*} \$ B \$ 6^{*} \$ B \$ 8^{*} D 25^{*}$ Relocation! $\mathrm{H} 25$ \\
\hline 26 & Pu-240 & $=$ Relocation!Y26 & $=\mathrm{C} 26 / \$ \mathrm{C} \$ 39$ & $=\$ B \$ 7^{*} \$ B \$ 3^{*} \$ B \$ 4^{*} \$ B \$ 5^{*} \$ B \$ 6^{*} \$ B \$ 8^{*} D 26^{*}$ Relocation! $\mathrm{H} 26$ \\
\hline 27 & Pu-241 & $=$ Relocation!Y27 & $=\mathrm{C} 27 / \$ \mathrm{C} \$ 39$ & $=\$ B \$ 7^{*} \$ B \$ 3^{*} \$ B \$ 4^{*} \$ B \$ 5^{*} \$ B \$ 6^{*} \$ B \$ 8^{*} D 27^{*}$ Relocation!H27 \\
\hline 28 & Pu-242 & $=$ Relocation!Y28 & $=\mathrm{C} 28 / \$ \mathrm{C} \$ 39$ & $=\$ B \$ 7^{*} \$ B \$ 3^{*} \$ B \$ 4^{*} \$ B \$ 5^{*} \$ B \$ 6^{*} \$ B \$ 8^{*} D 28^{*}$ Relocation! $\mathrm{H} 28$ \\
\hline 29 & Sr-90 & $=$ Relocation!Y29 & $=\mathrm{C} 29 / \$ \mathrm{C} \$ 39$ & $=\$ B \$ 7^{*} \$ B \$ 3^{*} \$ B \$ 4^{*} \$ B \$ 5^{*} \$ B \$ 6^{*} \$ B \$ 8^{*} D 29^{*}$ Relocation! $\mathrm{H} 29$ \\
\hline 30 & Tc-99 & $=$ Relocation!Y30 & $=\mathrm{C} 30 / \$ \mathrm{C} \$ 39$ & $=\$ B \$ 7^{*} \$ B \$ 3^{*} \$ B \$ 4^{*} \$ B \$ 5^{*} \$ B \$ 6^{*} \$ B \$ 8^{*} D 30^{*}$ Relocation!H30 \\
\hline$\overline{31}$ & Th-232 & $=$ Relocation!Y31 & $=\mathrm{C} 31 / \$ \mathrm{C} \$ 39$ & $=\$ B \$ 7^{*} \$ B \$ 3^{*} \$ B \$ 4^{*} \$ B \$ 5^{*} \$ B \$ 6^{*} \$ B \$ 8^{*} D 31^{*}$ Relocation! $H 31$ \\
\hline 32 & TI-204 & $=$ Relocation!Y32 & $=\mathrm{C} 32 / \$ \mathrm{C} \$ 39$ & $=\$ B \$ 7^{*} \$ B \$ 3^{*} \$ B \$ 4^{*} \$ B \$ 5^{*} \$ B \$ 6^{*} \$ B \$ 8^{*} D 32^{*}$ Relocation! $\mathrm{H} 32$ \\
\hline 33 & U-235 & $=$ Relocation!Y33 & $=\mathrm{C} 33 / \$ C \$ 39$ & $=\$ B \$ 7^{*} \$ B \$ 3^{*} \$ B \$ 4^{*} \$ B \$ 5^{*} \$ B \$ 6^{*} \$ B \$ 8^{*} D 33^{*}$ Relocation!H33 \\
\hline 34 & U-238 & $=$ Relocation!Y34 & $=\mathrm{C} 34 / \$ \mathrm{C} \$ 39$ & $=\$ B \$ 7^{*} \$ B \$ 3^{*} \$ B \$ 4^{*} \$ B \$ 5^{*} \$ B \$ 6^{*} \$ B \$ 8^{*} D 34^{*}$ Relocation! $\mathrm{H} 34$ \\
\hline 35 & & & & \\
\hline 36 & Ba-137m & $=$ Relocation!Y36 & $=\mathrm{C} 36 / \$ C \$ 39$ & \\
\hline 37 & $Y-90$ & $=$ Relocation!Y37 & $=\mathrm{C} 37 / \$ \mathrm{C} \$ 39$ & \\
\hline 38 & & & & \\
\hline 39 & Total & $=S U M(C 10: C 38)$ & $=S U M(D 10: D 38)$ & $=S U M(F 10: F 38)$ \\
\hline
\end{tabular}




\begin{tabular}{|c|c|c|c|}
\hline & $\mathrm{G}$ & $\mathrm{H}$ & 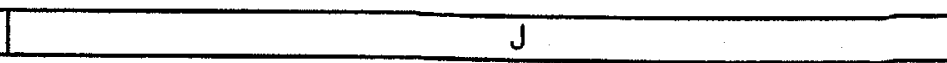 \\
\hline 9 & External EDE (mrem per uCi/m^2) & Skin Dose (mrem per uCi/m^2) & BS Dose (mrem per uCl/m^2) \\
\hline 10 & $=\$ B \$ 7^{*} \$ B \$ 8^{*} \$ D 10^{*}$ Relocation!E10 & $=\$ B \$ 7^{*} \$ B \$ 8^{*} \$ D 10^{*}$ Relocation $\$ F 10$ & $=\$ B \$ 7^{*} \$ B \$ 3^{*} \$ B \$ 4^{*} \$ B \$ 5^{*} \$ B \$ 6^{*} \$ B \$ 8^{*} \$ D 10^{*}$ Relocation $! \$ J 10$ \\
\hline 11 & $=\$ B \$ 7^{*} \$ B \$ 8^{*} \$ D 11^{*}$ Relocation!E11 & $=\$ B \$ 7^{*} \$ B \$ 8^{*} \$ D 11^{*}$ Relocation! $\$ F 11$ & $=\$ B \$ 7^{*} \$ B \$ 3^{*} \$ B \$ 4^{*} \$ B \$ 5^{*} \$ B \$ 6^{*} \$ B \$ 8^{*} \$ D 11^{*}$ Relocation! $\$ J 11$ \\
\hline 12 & $=\$ B \$ 7^{*} \$ B \$ 8^{*} \$ D 12^{*}$ Relocation!E12 & $=\$ B \$ 7^{*} \$ B \$ 8^{*} \$ D 12^{*}$ Relocation $! \$ F 12$ & $=\$ B \$ 7^{*} \$ B \$ 3^{*} \$ B \$ 4^{*} \$ B \$ 5^{*} \$ B \$ 6^{*} \$ B \$ 8^{*} \$ D 12^{*}$ Relocation! $\$ J 12$ \\
\hline 13 & $=\$ B \$ 7^{*} \$ B \$ 8^{*} \$ D 13^{*}$ Relocation!E13 & $=\$ B \$ 7^{*} \$ B \$ 8^{*} \$ D 13^{*}$ Relocation! $\$ F 13$ & $=\$ B \$ 7^{*} \$ B \$ 3^{*} \$ B \$ 4^{*} \$ B \$ 5^{*} \$ B \$ 6^{*} \$ B \$ 8^{*} \$ D 13^{*}$ Relocation! $\$ J 13$ \\
\hline 14 & $=\$ B \$ 7^{*} \$ B \$ 8^{*} \$ D 14^{*}$ Relocation!E14 & $=\$ B \$ 7^{*} \$ B \$ 8^{*} \$ D 14^{*}$ Relocation! $\$ F 14$ & $=\$ B \$ 7^{*} \$ B \$ 3^{*} \$ B \$ 4^{*} \$ B \$ 5^{*} \$ B \$ 6^{*} \$ B \$ 8^{*} \$ D 14^{*}$ Relocation! $\$ J 14$ \\
\hline 15 & $=\$ B \$ 7^{*} \$ B \$ 8 * \$ D 15^{*}$ Relocation!E15 & $=\$ B \$ 7^{*} \$ B \$ 8^{*} \$ D 15^{*}$ Relocation! $\$ F 15$ & $=\$ B \$ 7^{*} \$ B \$ 3^{*} \$ B \$ 4^{*} \$ B \$ 5^{*} \$ B \$ 6^{*} \$ B \$ 8^{*} \$ D 15^{*}$ Relocation! $\$ J 15$ \\
\hline 16 & $=\$ \mathrm{~B} \$ 7^{*} \$ \mathrm{~B} \$ 8^{*} \$ \mathrm{D} 16^{*}$ Relocation!E16 & $=\$ B \$ 7^{*} \$ B \$ 8^{*} \$ D 16^{*}$ Relocation! $\$ F 16$ & $=\$ B \$ 7^{*} \$ B \$ 3^{*} \$ B \$ 4^{*} \$ B \$ 5^{*} \$ B \$ 6^{*} \$ B \$ 8^{*} \$ D 16^{*}$ Relocation! $\$ J 16$ \\
\hline 17 & $=\$ B \$ 7^{*} \$ B \$ 8^{*} \$ D 17^{*}$ Relocation!E17 & $=\$ B \$ 7^{*} \$ B \$ 8^{*} \$ D 17^{*}$ Relocation! $\$ F 17$ & $=\$ B \$ 7^{*} \$ B \$ 3^{*} \$ B \$ 4^{*} \$ B \$ 5^{*} \$ B \$ 6^{*} \$ B \$ 8^{*} \$ D 17^{*}$ Relocation! $\$ J 17$ \\
\hline 18 & $=\$ B \$ 7^{*} \$ B \$ 8^{*} \$ D 18^{*}$ Relocation!E18 & $=\$ B \$ 7^{*} \$ B \$ 8^{*} \$ D 18 *$ Relocation! $\$ F 18$ & $=\$ B \$ 7^{*} \$ B \$ 3^{*} \$ B \$ 4^{*} \$ B \$ 5^{*} \$ B \$ 6^{*} \$ B \$ 8^{*} \$ D 18^{*}$ Relocation! $\$ J 18$ \\
\hline 19 & $=\$ B \$ 7^{*} \$ B \$ 8^{*} \$ D 19^{*}$ Relocation!E19 & $=\$ B \$ 7^{*} \$ B \$ 8^{*} \$ D 19^{*}$ Relocation! $\$ F 19$ & $=\$ B \$ 7^{*} \$ B \$ 3^{*} \$ B \$ 4^{*} \$ B \$ 5^{*} \$ B \$ 6^{*} \$ B \$ 8^{*} \$ D 19^{*}$ Relocation! $\$ 119$ \\
\hline 20 & $=\$ B \$ 7^{*} \$ B \$ 8^{*} \$ D 20^{*}$ Relocation!E20 & $=\$ B \$ 7^{*} \$ B \$ 8 * \$ D 20 *$ Relocation $! \$ F 20$ & $=\$ B \$ 7^{*} \$ B \$ 3^{*} \$ B \$ 4^{*} \$ B \$ 5^{*} \$ B \$ 6^{*} \$ B \$ 8^{*} \$ D 20^{*}$ Relocation $\$ J 20$ \\
\hline 21 & $=\$ B \$ 7^{*} \$ B \$ 8^{*} \$ D 21^{*}$ Relocation!E21 & $=\$ B \$ 7^{*} \$ B \$ 8 * \$ D 21 *$ Relocation $\$ \$ F 21$ & $=\$ B \$ 7^{*} \$ B \$ 3^{*} \$ B \$ 4^{*} \$ B \$ 5^{*} \$ B \$ 6^{*} \$ B \$ 8^{*} \$ D 21^{*}$ Relocation $\$ J 21$ \\
\hline 22 & $=\$ B \$ 7^{*} \$ B \$ 8^{*} \$ D 22^{*}$ Relocation!E22 & $=\$ \mathrm{~B} \$ 7^{*} \$ \mathrm{~B} \$ 8^{*} \$ \mathrm{D} 22^{*}$ Relocation! $\$ \mathrm{~F} 22$ & $=\$ B \$ 7^{*} \$ B \$ 3^{*} \$ B \$ 4^{*} \$ B \$ 5^{*} \$ B \$ 6^{*} \$ B \$ 8^{*} \$ D 22^{*}$ Relocation! $\$ J 22$ \\
\hline 23 & $=\$ B \$ 7^{*} \$ B \$ 8^{*} \$ D 23^{*}$ Relocation!E23 & $=\$ \mathrm{~B} \$ 7^{*} \$ \mathrm{~B} \$ 8^{*} \$ \mathrm{D} 23^{*}$ Relocation $\$ \mathrm{~F} 23$ & $=\$ B \$ 7^{*} \$ B \$ 3^{*} \$ B \$ 4^{*} \$ B \$ 5^{*} \$ B \$ 6^{*} \$ B \$ 8^{*} \$ D 23^{*}$ Relocation! $\$ \mathrm{~J} 23$ \\
\hline 24 & $=\$ B \$ 7^{*} \$ B \$ 8^{*} \$ D 24^{*}$ Relocation!E24 & $=\$ B \$ 7^{*} \$ B \$ 8^{*} \$ D 24^{*}$ Relocation! $\$ F 24$ & $=\$ B \$ 7^{*} \$ B \$ 3^{*} \$ B \$ 4^{*} \$ B \$ 5^{*} \$ B \$ 6^{*} \$ B \$ 8^{*} \$ D 24^{*}$ Relocation! $\$ J 24$ \\
\hline 25 & $=\$ B \$ 7^{*} \$ B \$ 8^{*} \$ D 25^{\star}$ Relocation!E25 & $=\$ B \$ 7^{*} \$ B \$ 8^{*} \$ D 25^{*}$ Relocation! $\$ F 25$ & $=\$ B \$ 7^{*} \$ B \$ 3^{*} \$ B \$ 4^{*} \$ B \$ 5^{*} \$ B \$ 6^{*} \$ B \$ 8^{*} \$ D 25^{*}$ Relocation! $\$ J 25$ \\
\hline 26 & $=\$ B \$ 7^{*} \$ B \$ 8^{*} \$ D 26^{*}$ Relocation!E26 & $=\$ B \$ 7^{*} \$ B \$ 8^{*} \$ D 26^{*}$ Relocation! $\$ F 26$ & 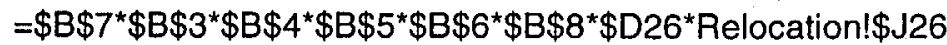 \\
\hline 27 & $=\$ B \$ 7^{*} \$ B \$ 8^{*} \$ D 27^{*}$ Relocation!E27 & $=\$ B \$ 7^{*} \$ B \$ 8^{*} \$ D 27^{*}$ Relocation! $\$ F 27$ & $=\$ B \$ 7^{*} \$ B \$ 3^{*} \$ B \$ 4^{*} \$ B \$ 5^{*} \$ B \$ 6^{*} \$ B \$ 8^{*} \$ D 27^{*}$ Relocation! $\$ J 27$ \\
\hline 28 & $=\$ B \$ 7^{*} \$ B \$ 8^{*} \$ D 28^{*}$ Relocation!E28 & $=\$ B \$ 7^{*} \$ B \$ 8^{*} \$ D 28 *$ Relocation! $\$ F 28$ & $=\$ B \$ 7^{*} \$ B \$ 3^{*} \$ B \$ 4^{*} \$ B \$ 5^{*} \$ B \$ 6^{*} \$ B \$ 8^{*} \$ D 28^{*}$ Relocation! $\$ J 28$ \\
\hline 29 & $=\$ B \$ 7^{*} \$ B \$ 8^{*} \$ D 29^{*}$ Relocation!E29 & $=\$ B \$ 7^{*} \$ B \$ 8^{*} \$ D 29^{*}$ Relocation! $\$ F 29$ & $=\$ B \$ 7^{*} \$ B \$ 3^{*} \$ B \$ 4^{*} \$ B \$ 5^{*} \$ B \$ 6^{*} \$ B \$ 8^{*} \$ D 29^{*}$ Relocation! $\$ J 29$ \\
\hline 30 & $=\$ B \$ 7^{*} \$ B \$ 8^{*} \$ D 30^{*}$ Relocation!E30 & $=\$ B \$ 7^{*} \$ B \$ 8^{*} \$ D 30^{*}$ Relocation! $\$ F 30$ & $=\$ B \$ 7^{*} \$ B \$ 3^{*} \$ B \$ 4^{*} \$ B \$ 5^{*} \$ B \$ 6^{*} \$ B \$ 8^{*} \$ D 30^{*}$ Relocation! $\$ J 30$ \\
\hline 31 & $=\$ B \$ 7^{*} \$ B \$ 8^{*} \$ D 31^{*}$ Relocation!E31 & $=\$ B \$ 7^{*} \$ B \$ 8^{*} \$ D 31^{*}$ Relocation!\$F31 & $=\$ B \$ 7^{*} \$ B \$ 3^{*} \$ B \$ 4^{*} \$ B \$ 5^{*} \$ B \$ 6^{*} \$ B \$ 8^{*} \$ D 31^{*}$ Relocation! $\$ J 31$ \\
\hline 32 & $=\$ B \$ 7^{*} \$ B \$ 8^{*} \$ D 32^{*}$ Relocation!E32 & $=\$ B \$ 7^{*} \$ B \$ 8^{*} \$ D 32 *$ Relocation! $\$ F 32$ & $=\$ B \$ 7^{*} \$ B \$ 3^{*} \$ B \$ 4^{*} \$ B \$ 5^{*} \$ B \$ 6^{*} \$ B \$ 8^{*} \$ D 32^{*}$ Relocation! $\$ J 32$ \\
\hline 33 & $=\$ B \$ 7^{*} \$ B \$ 8^{*} \$ D 33^{*}$ Relocation!E33 & $=\$ B \$ 7^{*} \$ B \$ 8^{*} \$ D 33^{*}$ Relocation! $\$ F 33$ & $=\$ B \$ 7^{*} \$ B \$ 3^{*} \$ B \$ 4^{*} \$ B \$ 5^{*} \$ B \$ 6^{*} \$ B \$ 8^{*} \$ D 33^{*}$ Relocation! $\$ J 33$ \\
\hline 34 & $=\$ B \$ 7^{*} \$ B \$ 8^{*} \$ D 34^{*}$ Relocation!E34 & $=\$ B \$ 7^{*} \$ B \$ 8^{*} \$ D 34^{*}$ Relocation! $\$ F 34$ & $=\$ B \$ 7^{*} \$ B \$ 3^{*} \$ B \$ 4^{*} \$ B \$ 5^{*} \$ B \$ 6^{*} \$ B \$ 8^{*} \$ D 34^{*}$ Relocation! $\$ J 34$ \\
\hline 35 & & & \\
\hline 36 & & & \\
\hline 37 & & & \\
\hline 38 & & & \\
\hline 39 & $=$ SUM(G10:G38) & $=S U M(110: 138)$ & $=S U M(J 10: J 38)$ \\
\hline
\end{tabular}




\begin{tabular}{|c|c|c|c|}
\hline & $E$ & F & G \\
\hline 44 & $5.11 E+00$ & DRL $\left(u C i / m^{\wedge} 2\right)--P A G /(C E D E+E x t e r n a l)$ \\
445 & $2.82 E+00$ & DRL (uCi/m^2) -- PAG/(BS Dose + External) \\
\cline { 1 - 1 } 46 & $6.35 E+05$ & DRL (uCi/m^2) -- PAG/(Skin Dose) \\
\hline 47 & & & \\
\hline
\end{tabular}




\begin{tabular}{|c|c|c|c|}
\hline & $E$ & $F$ & $\mathbf{G}$ \\
\hline 44 & $=G 3 /(F 39+G 39)$ & DRL (uCi/m^2) -- PAG/(CEDE+External) & \\
\hline 45 & $=\mathrm{G} 4 /(\mathrm{J} 39+\mathrm{G} 39)$ & DRL (uCi/m^2) -- PAG/(BS Dose + External) & \\
\hline 46 & $=\mathrm{G} 5 / 139$ & DRL (uCi/m^2) -- PAG/(Skin Dose) & \\
\hline 47 & & & \\
\hline
\end{tabular}




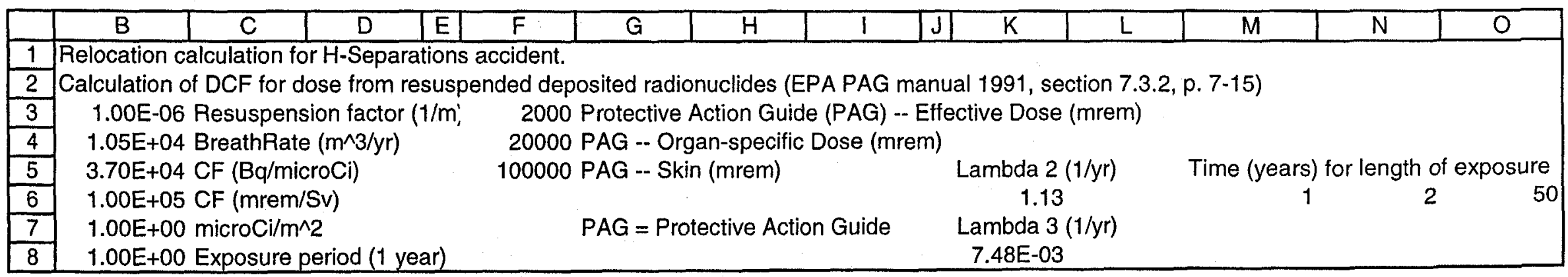




\begin{tabular}{|c|c|c|c|c|c|c|c|c|c|c|c|}
\hline & $\mathrm{B}$ & $\mathrm{C}$ & D & $E$ & $\mathrm{G}$ & $\mathrm{H}$ & 1 & $\mathrm{~J}$ & $\mathrm{~K}$ & $L$ & $M$ \\
\hline 9 & Nuclide & $\begin{array}{l}\text { Release } \\
(\mathrm{Ci})\end{array}$ & $\begin{array}{l}\text { Activity } \\
\text { fraction }\end{array}$ & $\begin{array}{l}\text { CEDE } \\
\text { (mrem per } \\
\mathrm{uCl} / \mathrm{m}^{\wedge} 2 \text { ) }\end{array}$ & $\begin{array}{l}\text { External } \\
\text { EDE } \\
\text { (mrem per } \\
\mathrm{uCi} / \mathrm{m}^{\wedge} 2 \text { ) }\end{array}$ & & $\begin{array}{l}\text { Skin Dose } \\
\text { (mrem per } \\
\text { uCi/m^2) }\end{array}$ & $\begin{array}{l}\text { BS Dose } \\
\text { (mrem per } \\
\mathrm{uCl} / \mathrm{m}^{\wedge} 2 \text { ) }\end{array}$ & $\begin{array}{l}\text { Halflife } \\
\text { (years) }\end{array}$ & Lambda1 & $\begin{array}{l}\text { Weathering } \\
\text { Factor ( } 1 \\
\text { year) }\end{array}$ \\
\hline 10 & $A m-241$ & 6.57E-01 & 1.87E-02 & $6.50 \mathrm{E}+01$ & 4.17E-02 & & 8.91E-02 & $1.18 \mathrm{E}+03$ & 458 & 0.001513 & $7.455 \mathrm{E}-01$ \\
\hline 11 & $A m-243$ & $0.00 \mathrm{E}+00$ & $0.00 E+00$ & $0.00 \mathrm{E}+00$ & $0.00 \mathrm{E}+00$ & & $0.00 E+00$ & $0.00 E+00$ & 7950 & 8.72E-05 & 7.460 E- 01 \\
\hline 12 & $\mathrm{Ba}-133$ & $0.00 E+00$ & $0.00 E+00$ & $0.00 E+00$ & $0.00 \mathrm{E}+00$ & & $0.00 E+00$ & $0.00 E+00$ & 10.5 & 0.066 & $7.242 E-01$ \\
\hline 13 & $\mathrm{Ce}-144$ & $0.00 E+00$ & $0.00 E+00$ & $0.00 E+00$ & $0.00 \mathrm{E}+00$ & & $0.00 E+00$ & $0.00 \mathrm{E}+00$ & 0.778082 & 0.890651 & 5.146E-01 \\
\hline 14 & Cf-249 & $0.00 E+00$ & $0.00 \mathrm{E}+00$ & $0.00 E+00$ & $0.00 \mathrm{E}+00$ & & $0.00 E+00$ & $0.00 E+00$ & 360 & 0.001925 & $7.454 \mathrm{E}-01$ \\
\hline 15 & Cf-252 & $0.00 E+00$ & $0.00 \mathrm{E}+00$ & $0.00 E+00$ & $0.00 \mathrm{E}+00$ & & $0.00 E+00$ & $0.00 \mathrm{E}+00$ & 2.646 & 0.261905 & 6.644E-01 \\
\hline 16 & $\mathrm{Cm}-244$ & $0.00 E+00$ & $0.00 E+00$ & $0.00 E+00$ & $0.00 E+00$ & & $0.00 E+00$ & $0.00 E+00$ & 17.6 & 0.039375 & 7.329E-01 \\
\hline 17 & $\mathrm{Cm}-246$ & $0.00 E+00$ & $0.00 E+00$ & $0.00 E+00$ & $0.00 \mathrm{E}+00$ & & $0.00 E+00$ & $0.00 E+00$ & 5500 & 0.000126 & 7.460E-01 \\
\hline 18 & Co-60 & $0.00 \mathrm{E}+00$ & $0.00 E+00$ & $0.00 E+00$ & $0.00 E+00$ & & $0.00 E+00$ & $0.00 E+00$ & 5.26 & 0.131749 & 7.033E-01 \\
\hline 19 & Cs-137 & $0.00 E+00$ & $0.00 E+00$ & $0.00 E+00$ & $0.00 E+00$ & & $0.00 \mathrm{E}+00$ & $0.00 E+00$ & 30 & 0.0231 & 7.383E-01 \\
\hline 20 & $\mathrm{H}-3$ & $0.00 \mathrm{E}+00$ & $0.00 \mathrm{E}+00$ & $0.00 E+00$ & $0.00 E+00$ & & $0.00 \mathrm{E}+00$ & $0.00 E+00$ & 12.3 & 0.056341 & 7.273E-01 \\
\hline 21 & $\mathrm{~Np}-237$ & $0.00 E+00$ & $0.00 E+00$ & $0.00 \mathrm{E}+00$ & $0.00 E+00$ & & $0.00 E+00$ & $0.00 E+00$ & 2140000 & $3.24 E-07$ & $7.460 \mathrm{E}-01$ \\
\hline 22 & $\mathrm{Pm}-147$ & $0.00 E+00$ & $0.00 E+00$ & $0.00 \mathrm{E}+00$ & $0.00 \mathrm{E}+00$ & & $0.00 E+00$ & $0.00 E+00$ & 2.62 & 0.264504 & 6.637E-01 \\
\hline 23 & $\mathrm{Pu}-238$ & $1.62 E+00$ & 4.61E-02 & $1.41 E+02$ & 2.94E-03 & & 2.46E-02 & $2.53 E+03$ & 86.4 & 0.008021 & 7.433E-01 \\
\hline 24 & Pu-239 & 4.23E-03 & $1.20 \mathrm{E}-04$ & 4.05E-01 & 3.39E-06 & & 2.51E-05 & $7.36 \mathrm{E}+00$ & 24390 & $2.84 E-05$ & $7.460 \mathrm{E}-01$ \\
\hline 25 & Pu-239/241 & $0.00 \mathrm{E}+00$ & $0.00 \mathrm{E}+00$ & & & & & & & & \\
\hline 26 & $\mathrm{Pu}-240$ & 2.43E-01 & $6.92 \mathrm{E}-03$ & $2.32 E+01$ & 4.23E-04 & & 3.52E-03 & $4.23 E+02$ & 6580 & 0.000105 & $7.460 \mathrm{E}-01$ \\
\hline 27 & Pu-241 & $3.26 E+01$ & $9.28 \mathrm{E}-01$ & $5.86 E+01$ & $0.00 E+00$ & & $0.00 E+00$ & $1.10 \mathrm{E}+03$ & 13.2 & 0.0525 & 7.286E-01 \\
\hline 28 & $\mathrm{Pu}-242$ & 1.54E-02 & 4.38E-04 & $1.41 E+00$ & 2.23E-05 & & $1.85 \mathrm{E}-04$ & $2.55 E+01$ & 379000 & $1.83 E-06$ & 7.460E-01 \\
\hline 29 & Sr-90 & $0.00 E+00$ & $0.00 \mathrm{E}+00$ & $0.00 E+00$ & $0.00 \mathrm{E}+00$ & & $0.00 E+00$ & $0.00 E+00$ & 28.1 & 0.024662 & 7.378E-01 \\
\hline 30 & TC-99 & $0.00 E+00$ & $0.00 E+00$ & $0.00 E+00$ & $0.00 E+00$ & & $0.00 E+00$ & $0.00 E+00$ & 212000 & 3.27E-06 & 7.460E-01 \\
\hline 31 & Th-232 & $0.00 \mathrm{E}+00$ & $0.00 E+00$ & $0.00 E+00$ & $0.00 \mathrm{E}+00$ & & $0.00 \mathrm{E}+00$ & $0.00 E+00$ & $1.4 \mathrm{E}+10$ & $4.95 E-11$ & 7.460E-01 \\
\hline 32 & |TI-204 & $0.00 E+00$ & $0.00 E+00$ & $0.00 E+00$ & $0.00 E+00$ & & $0.00 E+00$ & $0.00 E+00$ & 3.8 & 0.182368 & 6.878E-01 \\
\hline 33 & U-235 & $0.00 \mathrm{E}+00$ & $0.00 E+00$ & $0.00 E+00$ & $0.00 \mathrm{E}+00$ & & $0.00 E+00$ & $0.00 E+00$ & $7.1 E+08$ & $9.76 \mathrm{E}-10$ & 7.460E-01 \\
\hline$\frac{34}{35}$ & U-238 & $0.00 E+00$ & $0.00 E+00$ & $0.00 E+00$ & $0.00 E+00$ & & $0.00 E+00$ & $0.00 E+00$ & $4.51 E+09$ & $1.54 \mathrm{E}-10$ & 7.460E-01 \\
\hline 36 & $\mathrm{Ba}-137 \mathrm{~m}$ & $0.00 E+00$ & $0.00 E+00$ & & & & & & 4.86E-06 & 142727.6 & 7.006E-06 \\
\hline 37 & |Y-90 & $0.00 E+00$ & $0.00 E+00$ & & & & & & 0.007317 & 94.7064 & $1.048 \mathrm{E}-02$ \\
\hline 38 & & & & & & & & & & & \\
\hline 39 & Total & $3.51 E+01$ & $1.00 \mathrm{E}+00$ & $2.90 \mathrm{E}+02$ & 4.51E-02 & & 1.17E-01 & $5.26 \mathrm{E}+03$ & & & \\
\hline
\end{tabular}




\begin{tabular}{|c|c|c|c|c|c|c|}
\hline & B & $\mathrm{C}$ & D & $F$ & G & 1 \\
\hline 9 & Nuclide & Release (Ci) & Activity fraction & CEDE (mrem per uCl/m^2) & External EDE (mrem per $\left.\mathrm{uCl} / \mathrm{m}^{\wedge} 2\right)$ & Skin Dose (mrem per uCi/m^2) \\
\hline 10 & Am-241 & $=$ Relocation $\mid Y 10$ & $=C 10 / \$ C \$ 39$ & $=\$ \mathrm{~B} \$ 7^{*} \$ \mathrm{~B} \$ 3^{*} \$ \mathrm{~B} \$ 4^{*} \$ \mathrm{~B} \$ 5^{*} \$ \mathrm{~B} \$ 6^{*} \mathrm{D} 10^{*}$ Relocation!H10*M10 & $=\$ B \$ 7^{*} \$ D 10^{*}$ Relocation!E10*M10 & $=\$ B \$ 7^{*} \$ D 10^{*}$ Relocation $\$ \$ F 10^{*} \mathrm{M} 10$ \\
\hline 11 & Am-243 & $=$ RelocationIY 11 & $=C 11 / \$ C \$ 39$ & $=\$ B \$ 7^{*} \$ B \$ 3^{*} \$ B \$ 4^{*} \$ B \$ 5^{*} \$ B \$ 6^{*} D 11^{*}$ Relocation!H11"M11 & $=\$ B \$ 7^{*} \$ D 11^{*}$ Relocation!E11*M11 & $=\$ B \$ 7 \times D 11^{*}$ Relocation $\mid \$ F 11^{*} M 11$ \\
\hline 12 & Ba-133 & $=$ Relocation $1 Y 12$ & $=C 12 / \$ C \$ 39$ & $=\$ B \$ 7^{*} \$ B \$ 3^{*} \$ B \$ 4^{*} \$ B \$ 5^{*} \$ B \$ 6^{*} D 12^{*}$ Relocation!H $12^{*} M 12$ & $=\$ B \$ 7^{*} \$ D 12^{*}$ Relocation!E12*M12 & $=\$ B \$ 7^{*} \$ D 12^{*}$ Relocation! $\$ F 12^{*} \mathrm{M} 12$ \\
\hline 13 & Ce-144 & $=$ Relocation!Y 13 & $=\mathrm{C} 13 / \$ \mathrm{C} \$ 39$ & $=\$ B \$ 7^{*} \$ B \$ 3^{*} \$ B \$ 4^{*} \$ B \$ 5^{*} \$ B \$ 6^{*} D 13^{*}$ Relocation!H13*M13 & $=\$ B \$ 7^{*} \$ D 13^{*}$ Relocation!E13*M13 & $=\$ B \$ 7^{*} \$ D 13^{*}$ Relocation!\$F $13^{*} \mathrm{M} 13$ \\
\hline 14 & CI-249 & $=$ Relocation!Y14 & $=\mathrm{C} 14 / \$ \mathrm{C} \$ 39$ & $=\$ B \$ 7^{*} \$ B \$ 3^{*} \$ B \$ 4^{*} \$ B \$ 5^{*} \$ B \$ 6^{*} D 14^{*}$ Relocation!H14*M14 & $=\$ B \$ 7^{*} \$ D 14^{*}$ Relocation!E14*M14 & $=\$ B \$ 7^{*} \$ D 14^{*}$ Relocation $\$ \$ 14^{*} M 14$ \\
\hline 15 & CI-252 & $=$ RelocationtY15 & $=\mathrm{C} 15 / \$ \mathrm{C} \$ 39$ & $=\$ B \$ 7^{*} \$ \mathrm{~B} \$ 3^{*} \$ \mathrm{~B} \$ 4^{*} \$ \mathrm{~B} \$ 5^{*} \$ \mathrm{~B} \$ 6^{*} \mathrm{D} 15^{*}$ Relocation!H15*M15 & $=\$ B \$ 7^{*} \$ D 15^{*}$ Relocation!E15*M15 & $=\$ B \$ 7^{*} \$ D 15^{*}$ Relocation $\$$ F $15^{*} \mathrm{M} 15$ \\
\hline 16 & $\mathrm{Cm}-244$ & $=$ Relocation!Y 16 & $=C+6 / \$ C \$ 39$ & $=\$ B \$ 7^{*} \$ B \$ 3^{*} \$ B \$ 4^{*} \$ B \$ 5^{*} \$ B \$ 6^{*} D 16^{*}$ Relocation!H16* $M 16$ & $=\$ B \$ 7^{*} \$ D 16^{*}$ Relocation!E16*M16 & $=\$ B \$ 7^{*} \$ D 16^{*}$ Relocation! $\$ F 16^{*} M 16$ \\
\hline 17 & $\mathrm{Cm}-246$ & $=$ Relocation $\mid \mathrm{Y} 17$ & $=C 17 / \$ C \$ 39$ & $=\$ B \$ 7^{*} \$ B \$ 3^{*} \$ B \$ 4^{*} \$ B \$ 5^{*} \$ B \$ 6^{*} D 17^{*}$ Relocation!H17*M17 & $=\$ B \$ 7^{*} \$ D 17^{*}$ Relocation!E17*M17 & $=\$ B \$ 7^{*} \$ D 17^{*}$ Relocation $\$ F 17^{*} M 17$ \\
\hline 18 & Co-60 & $=$ Relocation!Y18 & $=C 18 / \$ C \$ 39$ & $=\$ B \$ 7^{*} \$ B \$ 3^{*} \$ B \$ 4^{*} \$ B \$ 5^{*} \$ B \$ 6^{*} D 18^{*}$ Relocation!H $18^{*} M 18$ & $=\$ B \$ 7^{*} \$ D 18^{*}$ Relocation!E $18^{*} \mathrm{M} 18$ & $=\$ B \$ 7^{*} \$ D 18^{*}$ Relocation! $\$ F 18^{*} \mathrm{M} 18$ \\
\hline 19 & Cs-137 & $=$ Relocation!Y 19 & $=C 19 / \$ C \$ 39$ & $=\$ B \$ 7^{*} \$ B \$ 3^{*} \$ B \$ 4^{*} \$ B \$ 5^{*} \$ B \$ 6^{*} D 19^{*}$ Relocation!H19*M19 & $=\$ B \$ 7^{*} \$ D 19^{*}$ Relocation!E $19^{*} \mathrm{M} 19$ & $=\$ B \$ 7^{*} \$ \mathrm{D} 19^{*}$ Relocation! $\$ \mathrm{~F} 19^{*} \mathrm{M} 19$ \\
\hline 20 & $\mathrm{H}-3$ & $=$ Relocation!Y20 & $=\mathrm{C} 20 / \$ \mathrm{C} \$ 39$ & $=\$ B \$ 7^{*} \$ B \$ 3^{*} \$ B \$ 4^{*} \$ B \$ 5^{*} \$ B \$ 6^{*} D 20^{*}$ Relocation!H20*M2O & $=\$ B \$ 7^{*} \$ D 20^{*}$ Relocation!E20*M2O & $=\$ \mathrm{~B} \$ 7^{*} \$ \mathrm{D} 20^{*}$ Relocation! $\$ \mathrm{~F} 20^{*} \mathrm{M} 20$ \\
\hline 21 & $\mathrm{~Np}-237$ & $=$ Relocation!Y21 & $=\mathrm{C} 21 / \$ C \$ 39$ & $=\$ \mathrm{~B} \$ 7^{*} \$ \mathrm{~B} \$ 3^{*} \$ \mathrm{~B} \$ 4^{*} \$ \mathrm{~B} \$ 5^{*} \$ \mathrm{~B} \$ 6^{*} \mathrm{D} 21^{*}$ Relocation!H21*M21 & $=\$ B \$ 7^{*} \$ D 21^{*}$ Relocation!E21 ${ }^{*} \mathrm{M} 21$ & $=\$ \mathrm{~B} \$ 7^{*} \$ \mathrm{D} 21^{*}$ Relocation! $\$ \mathrm{~F} 21^{*} \mathrm{M} 21$ \\
\hline 22 & $\mathrm{Pm}=147$ & $=$ Relocation!Y22 & $=\mathrm{C} 22 / \$ \mathrm{C} \$ 39$ & $=\$ B \$ 7^{*} \$ B \$ 3^{*} \$ B \$ 4^{*} \$ B \$ 5^{*} \$ B \$ 6^{*} D 22^{*}$ RelocationlH22* M22 & $=\$ B \$ 7^{*} \$ \mathrm{D} 22^{*}$ Relocation!E22*M22 & $=\$ B \$ 7^{*} \$ D 22^{*}$ Relocation $1 \$ F 22^{*} \mathrm{M} 22$ \\
\hline 23 & Pu-238 & $=$ RelocationIY23 & $=C 23 / \$ C \$ 39$ & $=\$ \mathrm{~B} \$ 7^{*} \$ \mathrm{~B} \$ 3^{*} \$ \mathrm{~B} \$ 4^{*} \$ \mathrm{~B} \$ 5^{*} \$ \mathrm{~B} \$ 6^{*} \mathrm{D} 23^{*}$ Relocation!H $3^{*} \mathrm{M} 23$ & $=\$ B \$ 7^{*} \$ D 23^{*}$ Relocation!E23*M23 & $=\$ \mathrm{~B} \$ 7^{*} \$ \mathrm{D} 23^{*}$ Relocation! $\$ \mathrm{~F} 23^{*} \mathrm{M} 23$ \\
\hline 24 & Pu-239 & $=$ RelocationlY24 & $=C 24 / \$ C \$ 39$ & $=\$ B \$ 7^{*} \$ B \$ 3^{*} \$ B \$ 4^{*} \$ B \$ 5^{*} \$ B \$ 6^{*} D 24^{*}$ Relocation!H24* M24 & $=\$ B \$ 7^{*} \$ D 24^{*}$ Relocation!E24*M24 & $=\$ B \$ 7^{*} \$ D 24^{*}$ Relocation!\$F $24^{*} \mathrm{M} 24$ \\
\hline 25 & Pu-239/241 & $=$ Relocation!Y25 & $=\mathrm{C} 25 / \$ \mathrm{C} \$ 39$ & & & \\
\hline 26 & Pu-240 & $=$ Relocation!Y26 & $=\mathrm{C} 26 / \$ \mathrm{C} \$ 39$ & $=\$ \mathrm{~B} \$ 7^{*} \$ \mathrm{~B} \$ 3^{*} \$ \mathrm{~B} \$ 4^{*} \$ \mathrm{~B} \$ 5^{*} \$ \mathrm{~B} \$ 6^{*} \mathrm{D} 26^{*}$ RelocationlH26*M26 & $=\$ B \$ 7 * \$ D 26^{*}$ RelocationlE26*M26 & $=\$ B \$ 7^{*} \$ \mathrm{D} 26^{*}$ Relocation!\$F26*M26 \\
\hline 27 & Pu-241 & =Relocation!Y27 & $=\mathrm{C} 27 / \$ \mathrm{C} \$ 39$ & $=\$ B \$ 7^{*} \$ \mathrm{~B} \$ 3^{*} \$ \mathrm{~B} \$ 4^{*} \$ \mathrm{~B} \$ 5^{*} \$ \mathrm{~B} \$ 6^{*} \mathrm{D} 27^{*}$ Relocation!H27*M27 & $=\$ B \$ 7^{*} \$ D 27^{*}$ RelocationlE27*M27 & $=\$ B \$ 7^{*} \$ D 27^{*}$ Relocation!\$F27*M27 \\
\hline 28 & Pu-242 & $=$ RelocationiY28 & $=\mathrm{C} 28 / \$ \mathrm{C} \$ 39$ & $=\$ B \$ 7^{*} \$ B \$ 3^{*} \$ B \$ 4^{*} \$ B \$ 5^{*} \$ B \$ 6^{*} D 28^{*}$ RelocationlH28*M28 & $=\$ B \$ 7^{*} \$ D 28^{*}$ Relocation!E28*M28 & $=\$ B \$ 7^{*} \$ D 28^{*}$ Relocation! $\$ F 28^{*} \mathrm{M} 28$ \\
\hline 29 & Sr-90 & $=$ Relocation!Y29 & $=\mathrm{C} 29 / \$ \mathrm{C} \$ 39$ & $=\$ B \$ 7^{*} \$ B \$ 3^{*} \$ B \$ 4^{*} \$ B \$ 5^{*} \$ B \$ 6^{*} \mathrm{D} 29^{*}$ Relocation!H29*M29 & $=\$ B \$ 7^{*} \$ D 29^{*}$ Relocation!E29*M29 & $=\$ B \$ 7^{*} \$ D 29^{*}$ Relocation! $\$ F 29^{*} \mathrm{M} 29$ \\
\hline 30 & TC-99 & $=$ Relocation!Y30 & $=\mathrm{C} 30 / \$ \mathrm{C} \$ 39$ & $=\$ B \$ 7^{*} \$ B \$ 3^{*} \$ B \$ 4^{*} \$ B \$ 5^{*} \$ B \$ 6^{*} \mathrm{D} 30^{*}$ Relocation!H30*M30 & $=\$ B \$ 7 * \$ D 30^{*}$ Relocation!E30*M30 & $=\$ B \$ 7 * \$ D 30 *$ Relocation! $\$ F 30^{*} \mathrm{M} 30$ \\
\hline 31 & Th-232 & $=$ Relocation!Y31 & $=C 31 / \$ C \$ 39$ & $=\$ B \$ 7^{*} \$ B \$ 3^{*} \$ B \$ 4^{*} \$ B \$ 5^{*} \$ B \$ 6^{*} D 31^{*}$ Relocation! H31* $M 31$ & $=\$ B \$ 7^{*} \$ D 31^{*}$ Relocation!E31*M31 & $=\$ B \$ 7^{*} \$ D 31^{*}$ Relocation $\$ F 31^{*} \mathrm{M} 31$ \\
\hline 32 & TI-204 & $=$ Relocation!Y32 & $=\mathrm{C} 32 / \$ C \$ 39$ & $=\$ B \$ 7^{*} \$ \mathrm{~B} \$ 3^{*} \$ \mathrm{~B} \$ 4^{*} \$ \mathrm{~B} \$ 5^{*} \$ \mathrm{~B} \$ 6^{*} \mathrm{D} 32^{*}$ RelocationIH32*M32 & $=\$ B \$ 7^{*} \$ D 32 *$ Relocation!E32*M32 & $=\$ B \$ 7^{*} \$ \mathrm{D} 32^{*}$ Relocation $\$ \mathrm{~F} 32^{*} \mathrm{M} 32$ \\
\hline 33 & U-235 & $=$ Refocation!Y33 & $=\mathrm{C} 33 / \$ \mathrm{C} \$ 39$ & $=\$ B \$ 7^{*} \$ B \$ 3^{*} \$ B \$ 4^{*} \$ B \$ 5^{*} \$ B \$ 6^{*} \mathrm{D} 33^{*}$ Relocation!H33*M33 & $=\$ B \$ 7^{*} \$ D 33^{*}$ Relocation!E33*M33 & $=\$ \mathrm{~B} \$ 7^{*} \$ \mathrm{D} 33^{*}$ Relocation!\$F33*M33 \\
\hline 34 & $\mathrm{U}-238$ & $=$ Relocation $I Y 34$ & $=C 34 / \$ C \$ 39$ & $=\$ B \$ 7^{*} \$ B \$ 3^{*} \$ B \$ 4^{*} \$ B \$ 5^{*} \$ B \$ 6^{*} D 34^{*}$ Relocation!H34 ${ }^{*} M 34$ & $=\$ B \$ 7^{*} \$ D 34^{*}$ Relocation!E34*M34 & $=\$ B \$ 7^{*} \$ D 34^{*}$ Relocation!\$F34"M34 \\
\hline 35 & & & & & & \\
\hline 36 & Ba-137m & $=$ Relocation!Y36 & $=\mathrm{C} 36 / \$ C \$ 39$ & & & \\
\hline \begin{tabular}{|l|}
37 \\
38
\end{tabular} & Y-90 & $=$ RelocationIY37 & $=C 37 / \$ C \$ 39$ & & & \\
\hline 39 & Total & $=$ SUM(C10:C38) & $=S U M(D 10: D 38$ & $3_{i}^{\prime}=S U M(F 10: F 38)$ & $=S U M(G 10: G 38)$ & $=\operatorname{SUM}(110: 138)$ \\
\hline
\end{tabular}




\begin{tabular}{|c|c|c|c|}
\hline & C & $\bar{D}$ & $E$ \\
\hline 44 & $6.90 \mathrm{E}+00$ & DRL (uCi/m^2) -- PAG/(CEDE+External) & \\
\hline 45 & $3.80 \mathrm{E}+00$ & DRL $\left(u C i / m^{\wedge} 2\right)--$ PAG/(BS Dose + External) & \\
\hline 46 & $8.52 E+05$ & DRL (uCi/m^2) -- PAG/(Skin Dose) & \\
\hline 47 & & & \\
\hline
\end{tabular}




\begin{tabular}{|c|c|c|c|}
\hline & C & D & $E$ \\
\hline 44 & $=F 3 /(F 39+G 39)$ & DRL (uCi/m^2) -- PAG/(CEDE+External) & \\
\hline 45 & $=F 4 /(J 39+G 39)$ & DRL (uCi/m^2) -- PAG/(BS Dose + External) & \\
\hline 46 & $=F 5 / 139$ & DRL (uCi/m^2) -- PAG/(Skin Dose) & \\
\hline 47 & & & \\
\hline
\end{tabular}




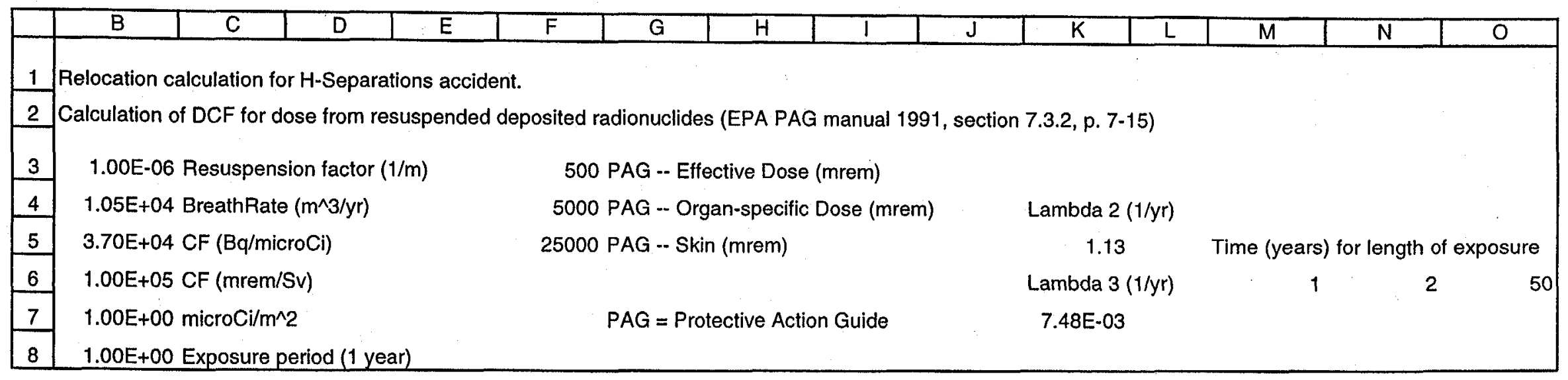




\begin{tabular}{|c|c|c|c|c|c|c|c|c|c|c|c|c|}
\hline & $B$ & $C$ & $\mathrm{D}$ & $E$ & $F$ & $\mathrm{G}$ & $\mathrm{H}$ & 1 & $J$ & K & $L$ & $N$ \\
\hline 9 & Nuclide & $\begin{array}{l}\text { Release } \\
\text { (Ci) }\end{array}$ & $\begin{array}{l}\text { Activity } \\
\text { fraction }\end{array}$ & & $\begin{array}{l}\text { CEDE } \\
\text { (mrem per } \\
\mathrm{uCl} / \mathrm{m}^{\wedge} 2 \text { ) }\end{array}$ & $\begin{array}{l}\text { External } \\
\text { EDE } \\
\text { (mrem per } \\
\mathrm{uCi} / \mathrm{m}^{\wedge} 2 \text { ) }\end{array}$ & & $\begin{array}{l}\text { Skin Dose } \\
\text { (mrem per } \\
\mathrm{uCi} / \mathrm{m}^{\wedge} 2 \text { ) }\end{array}$ & $\begin{array}{l}\text { BS Dose } \\
\text { (mrem per } \\
\mathrm{uCl} / \mathrm{m}^{\wedge} 2 \text { ) }\end{array}$ & $\begin{array}{l}\text { Halflife } \\
\text { (years) }\end{array}$ & Lambda1 & $\begin{array}{l}\text { Weathering } \\
\text { Factor ( } 2 \\
\text { years) }\end{array}$ \\
\hline 10 & Am-241 & 6.57E-01 & 1.87E-02 & & $1.07 E+02$ & $6.89 \mathrm{E}-02$ & & 1.47E-01 & $1.94 E+03$ & 458 & 0.001513 & $1.232 E+00$ \\
\hline 11 & $A m-243$ & $0.00 E+00$ & $0.00 E+00$ & & $0.00 \mathrm{E}+00$ & $0.00 E+00$ & & $0.00 \mathrm{E}+00$ & $0.00 \mathrm{E}+00$ & 7950 & 8.72E-05 & $1.234 E+00$ \\
\hline 12 & $\mathrm{Ba}-133$ & $0.00 E+00$ & $0.00 E+00$ & & $0.00 E+00$ & $0.00 E+00$ & & $0.00 E+00$ & $0.00 E+00$ & 10.5 & 0.066 & 1.167E+00 \\
\hline 13 & $\mathrm{Ce}-144$ & $0.00 E+00$ & $0.00 E+00$ & & $0.00 E+00$ & $0.00 E+00$ & & $0.00 E+00$ & $0.00 \mathrm{E}+00$ & 0.778082 & 0.890651 & $6.499 \mathrm{E}-01$ \\
\hline 14 & $C f-249$ & $0.00 E+00$ & $0.00 \mathrm{E}+00$ & & $0.00 E+00$ & $0.00 \mathrm{E}+00$ & & $0.00 E+00$ & $0.00 E+00$ & 360 & 0.001925 & $1.232 \mathrm{E}+00$ \\
\hline 15 & Cf-252 & $0.00 E+00$ & $0.00 E+00$ & & $0.00 E+00$ & $0.00 E+00$ & & $0.00 E+00$ & $0.00 E+00$ & 2.646 & 0.261905 & $9.968 E-01$ \\
\hline 16 & $\mathrm{Cm}-244$ & $0.00 E+00$ & $0.00 E+00$ & & $0.00 E+00$ & $0.00 E+00$ & & $0.00 E+00$ & $0.00 \mathrm{E}+00$ & 17.6 & 0.039375 & $1.193 E+00$ \\
\hline 17 & $\mathrm{Cm}-246$ & $0.00 \mathrm{E}+00$ & $0.00 E+00$ & & $0.00 \mathrm{E}+00$ & $0.00 E+00$ & & $0.00 E+00$ & $0.00 \mathrm{E}+00$ & 5500 & 0.000126 & $1.234 \mathrm{E}+00$ \\
\hline 18 & Co-60 & $0.00 \mathrm{E}+00$ & $0.00 E+00$ & & $0.00 E+00$ & $0.00 E+00$ & & $0.00 E+00$ & $0.00 E+00$ & 5.26 & 0.131749 & $1.105 E+00$ \\
\hline 19 & Cs-137 & $0.00 E+00$ & $0.00 E+00$ & & $0.00 \mathrm{E}+00$ & $0.00 E+00$ & & $0.00 E+00$ & $0.00 E+00$ & 30 & 0.0231 & $1.210 E+00$ \\
\hline 20 & $\mathrm{H}-3$ & $0.00 E+00$ & $0.00 E+00$ & & $0.00 \mathrm{E}+00$ & $0.00 E+00$ & & $0.00 \mathrm{E}+00$ & $0.00 \mathrm{E}+00$ & 12.3 & 0.056341 & $1.176 E+00$ \\
\hline 21 & $\mathrm{~Np}-237$ & $0.00 E+00$ & $0.00 E+00$ & & $0.00 E+00$ & $0.00 E+00$ & & $0.00 E+00$ & $0.00 E+00$ & 2140000 & $3.24 \mathrm{E}-07$ & $1.234 \mathrm{E}+00$ \\
\hline 22 & $\mathrm{Pm}-147$ & $0.00 E+00$ & $0.00 E+00$ & & $0.00 \mathrm{E}+00$ & $0.00 \mathrm{E}+00$ & & $0.00 E+00$ & $0.00 E+00$ & 2.62 & 0.264504 & $9.948 \mathrm{E}-01$ \\
\hline 23 & Pu-238 & $1.62 E+00$ & 4.61E-02 & & $2.33 E+02$ & 4.85E-03 & & 4.05E-02 & 4.17E+03 & 86.4 & 0.008021 & $1.225 E+00$ \\
\hline 24 & Pu-239 & 4.23E-03 & $1.20 \mathrm{E}-04$ & & $6.69 \mathrm{E}-01$ & 5.61E-06 & & 4.16E-05 & $1.22 E+01$ & 24390 & 2.84E-05 & $1.234 \mathrm{E}+00$ \\
\hline 25 & Pu-239/241 & $0.00 E+00$ & $0.00 E+00$ & & & & & & & & & \\
\hline 26 & Pu-240 & 2.43E-01 & $6.92 E-03$ & & $3.84 E+01$ & $7.00 E-04$ & & 5.83E-03 & $6.99 E+02$ & 6580 & 0.000105 & $1.234 \mathrm{E}+00$ \\
\hline 27 & $\mathrm{Pu}-241$ & $3.26 \mathrm{E}+01$ & $9.28 E-01$ & & $9.48 \mathrm{E}+01$ & $0.00 \mathrm{E}+00$ & & $0.00 E+00$ & $1.79 E+03$ & 13.2 & 0.0525 & $1.180 E+00$ \\
\hline 28 & $\mathrm{Pu}-242$ & 1.54E-02 & 4.38E-04 & & $2.33 \mathrm{E}+00$ & 3.69E-05 & & 3.07E-04 & $4.22 E+01$ & 379000 & $1.83 \mathrm{E}-06$ & $1.234 E+00$ \\
\hline 29 & Sr-90 & $0.00 E+00$ & $0.00 E+00$ & & $0.00 \mathrm{E}+00$ & $0.00 \mathrm{E}+00$ & & $0.00 E+00$ & $0.00 E+00$ & 28.1 & 0.024662 & $1.208 \mathrm{E}+00$ \\
\hline 30 & Tc-99 & $0.00 E+00$ & $0.00 E+00$ & & $0.00 E+00$ & $0.00 E+00$ & & $0.00 \mathrm{E}+00$ & $0.00 \mathrm{E}+00$ & 212000 & 3.27E-06 & $1.234 \mathrm{E}+00$ \\
\hline 31 & Th-232 & $0.00 E+00$ & $0.00 E+00$ & & $0.00 E+00$ & $0.00 \mathrm{E}+00$ & & $0.00 E+00$ & $0.00 E+00$ & $1.4 E+10$ & $4.95 \mathrm{E}-11$ & $1.234 \mathrm{E}+00$ \\
\hline 32 & TI-204 & $0.00 E+00$ & $0.00 E+00$ & & $0.00 E+00$ & $0.00 E+00$ & & $0.00 E+00$ & $0.00 E+00$ & 3.8 & 0.182368 & $1.061 E+00$ \\
\hline 33 & U-235 & $0.00 E+00$ & $0.00 E+00$ & & $0.00 \mathrm{E}+00$ & $0.00 E+00$ & & $0.00 E+00$ & $0.00 E+00$ & $7.1 E+08$ & $9.76 \mathrm{E}-10$ & $1.234 E+00$ \\
\hline 34 & U-238 & $0.00 E+00$ & $0.00 E+00$ & & $0.00 E+00$ & $0.00 \mathrm{E}+00$ & & $0.00 E+00$ & $0.00 \mathrm{E}+00$ & $4.51 E+09$ & $1.54 \mathrm{E}-10$ & $1.234 \mathrm{E}+00$ \\
\hline 36 & Ba-137m & $0.00 E+00$ & $0.00 \mathrm{E}+00$ & & & & & & & 4.86E-06 & 142727.6 & 7.006E-06 \\
\hline 37 & $Y-90$ & $0.00 E+00$ & $0.00 E+00$ & & & & & & & 0.007317 & 94.7064 & $1.048 E-02$ \\
\hline 38 & & & & & & & & & & & & \\
\hline 39 & Total & $3.51 E+01$ & $1.00 E+00$ & & $4.76 E+02$ & $7.45 \mathrm{E}-02$ & & $1.94 E-01$ & $8.65 E+03$ & & & \\
\hline 40 & \multirow{2}{*}{\multicolumn{4}{|c|}{ Dose in second year following release: }} & & & & & & & & \\
\hline$\frac{41}{42}$ & & & & & $1.87 E+02$ & $2.94 E-02$ & & $7.65 \mathrm{E}-02$ & $3.39 E+03$ & & & \\
\hline
\end{tabular}




\begin{tabular}{|c|c|c|c|c|c|c|}
\hline & B & $\mathrm{C}$ & $\mathrm{D}$ & $F$ & G & 1 \\
\hline 9 & Nuclide & Release (Ci) & Activity fraction & CEDE (mrem per $\left.\mathrm{uCl} / \mathrm{m}^{\wedge} 2\right)$ & External EDE (mrem per uCi/m^2) & Skin Dose (mrem per uCi/m^2) \\
\hline 10 & Am-241 & $=$ RelocationIY10 & $=C 10 / \$ C \$ 39$ & $=\$ B \$ 7^{*} \$ B \$ 3^{*} \$ B \$ 4^{*} \$ B \$ 5^{*} \$ B \$ 6^{*} D 10^{*}$ Relocation!H10*N10 & $=\$ B \$ 7^{*} \$ D 10^{*}$ Relocation $I E 10^{*} \mathrm{~N} 10$ & $=\$ B \$ 7^{*} \$ D 10^{*}$ Relocation $1 \$ F 10^{*} N 10$ \\
\hline 11 & $A m-243$ & $=$ Relocation!Y11 & $=\mathrm{C} 11 / \$ \mathrm{C} \$ 39$ & $=\$ B \$ 7^{*} \$ B \$ 3^{*} \$ B \$ 4^{*} \$ B \$ 5^{*} \$ B \$ 6^{*} D 11^{*}$ RelocationlH11*N11 & $=\$ B \$ 7^{*} \$ D 11^{*}$ RelocationIE $11^{*} \mathrm{~N} 11$ & $=\$ B \$ 7^{*} \$ D 11 *$ Relocation!\$F11*N11 \\
\hline 12 & $\mathrm{Ba}-133$ & $=$ Relocation!Y12 & $=\mathrm{C} 12 / \$ \mathrm{C} \$ 39$ & $=\$ B \$ 7^{*} \$ B \$ 3^{*} \$ B \$ 4^{*} \$ B \$ 5^{*} \$ B \$ 6^{*} D 12^{*}$ Relocation!H12*N12 & $=\$ B \$ 7^{*} \$ D 12^{*}$ Relocation!E 12*N12 & $=\$ B \$ 7^{*} \$ D 12^{*}$ Relocation! $\$ F 12^{*} \mathrm{~N} 12$ \\
\hline 13 & $\mathrm{Ce}-144$ & $=$ RelocationIY13 & $=C 13 / \$ C \$ 39$ & $=\$ B \$ 7^{*} \$ B \$ 3^{*} \$ B \$ 4^{*} \$ B \$ 5^{*} \$ B \$ 6^{*} D 13^{*}$ Relocation!H13*N13 & $=\$ B \$ 7^{*} \$ D 13^{*}$ Relocation!E $13^{*} \mathrm{~N} 13$ & $=\$ B \$ 7^{*} \$ D 13^{*}$ Relocation! $\$ F 13^{*} N 13$ \\
\hline 14 & Cf-249 & $=$ Relocation!Y14 & $=C 14 / \$ C \$ 39$ & $=\$ B \$ 7^{*} \$ B \$ 3^{*} \$ B \$ 4^{*} \$ B \$ 5^{*} \$ B \$ 6^{*} D 14^{*}$ Relocation $1 \mathrm{H} 14^{*} \mathrm{~N} 14$ & $=\$ B \$ 7^{*} \$ D 14^{*}$ RelocationlE14*N14 & $=\$ B \$ 7^{*} \$ D 14^{*}$ Relocation! $\$ F 14^{*} \mathrm{~N} 14$ \\
\hline 15 & Cf-252 & $=$ Relocation $1 Y 15$ & $=C 15 / \$ C \$ 39$ & $=\$ B \$ 7^{*} \$ B \$ 3^{*} \$ B \$ 4^{*} \$ B \$ 5^{*} \$ B \$ 6^{*} D 15^{*}$ Relocation!H15*N15 & $=\$ B \$ 7^{*} \$ D 15^{*}$ Relocation!E $15^{*} \mathrm{~N} 15$ & $=\$ B \$ 7^{*} \$ D 15 *$ Relocation $\$ F 15^{*} N 15$ \\
\hline 16 & $\mathrm{Cm}-244$ & $=$ RelocationIY16 & $=\mathrm{C} 16 / \$ \mathrm{C} \$ 39$ & $=\$ B \$ 7^{*} \$ B \$ 3^{*} \$ B \$ 4^{*} \$ B \$ 5^{*} \$ B \$ 6^{*} D 16^{*}$ Relocation!H16*N16 & $=\$ B \$ 7^{\star} \$ D 16^{*}$ Relocation!E16*N16 & $=\$ B \$ 7^{*} \$ D 16^{*}$ Relocation! $\$ F 16^{*} \mathrm{~N} 16$ \\
\hline 17 & $\mathrm{Cm}-246$ & $=$ Relocation $\mid Y 17$ & $=\mathrm{C} 17 / \$ C \$ 39$ & $=\$ B \$ 7^{*} \$ B \$ 3^{*} \$ B \$ 4^{*} \$ B \$ 5^{*} \$ B \$ 6^{*} D 17^{*}$ Relocation!H17*N17 & $=\$ B \$ 7^{*} \$ D 17^{*}$ RelocationlE17*N17 & $=\$ B \$ 7^{*} \$ D 17^{*}$ Relocationi $\$ F 17^{*} \mathrm{~N} 17$ \\
\hline 18 & Co-60 & $=$ Relocation $1 Y 18$ & $=C 18 / \$ C \$ 39$ & $=\$ B \$ 7^{*} \$ B \$ 3^{*} \$ B \$ 4^{*} \$ B \$ 5^{*} \$ B \$ 6^{*} D 18^{*}$ Relocation!H18*N18 & $=\$ B \$ 7^{*} \$ D 18^{*}$ Relocation!E18*N18 & $=\$ B \$ 7 * \$ D 18^{*}$ Relocation $1 \$ F 18^{*} N 18$ \\
\hline 19 & Cs-137 & $=$ Relocation!Y19 & $=C 19 / \$ C \$ 39$ & $=\$ B \$ 7^{*} \$ B \$ 3^{*} \$ B \$ 4^{*} \$ B \$ 5^{*} \$ B \$ 6^{*} D 19^{*}$ Relocation!H19*N19 & $=\$ \mathrm{~B} \$ 7^{*} \$ \mathrm{D} 19^{*}$ RelocationlE19*N19 & $=\$ B \$ 7 * \$ D 19^{*}$ Relocationl\$F19*N19 \\
\hline 20 & $\mathrm{H}-3$ & $=$ Relocation!Y20 & $=\mathrm{C} 20 / \$ \mathrm{C} \$ 39$ & $=\$ B \$ 7^{*} \$ \mathrm{~B} \$ 3^{*} \$ \mathrm{~B} \$ 4^{*} \$ \mathrm{~B} \$ 5^{*} \$ \mathrm{~B} \$ 6^{*} \mathrm{D} 20^{*}$ Relocation!H $20^{*} \mathrm{~N} 20$ & $=\$ B \$ 7 * \$ D 20^{*}$ Relocation!E20*N20 & $=\$ B \$ 7^{*} \$ D 20^{*}$ Relocation $1 \$ \mathrm{~F} 20^{*} \mathrm{~N} 20$ \\
\hline 21 & Np-237 & $=$ Relocation!Y21 & $=\mathrm{C} 21 / \$ \mathrm{C} \$ 39$ & $=\$ B \$ 7^{*} \$ B \$ 3^{*} \$ B \$ 4^{*} \$ B \$ 5^{*} \$ B \$ 6^{*} \mathrm{D} 21^{*}$ Relocation!H21*N21 & $=\$ B \$ 7^{*} \$ D 21 *$ Relocation!E21*N21 & $=\$ B \$ 7 * \$ D 21^{*}$ Relocation! $\$ F 21 * N 21$ \\
\hline 22 & $\mathrm{Pm}-147$ & $=$ Relocation!Y22 & $=\mathrm{C} 22 / \$ \mathrm{C} \$ 39$ & $=\$ B \$ 7^{*} \$ B \$ 3^{*} \$ B \$ 4^{*} \$ B \$ 5^{*} \$ B \$ 6^{*} \mathrm{D} 22^{*}$ Relocation!H22*N22 & $=\$ B \$ 7 * \$ D 22 *$ Relocation!E22*N22 & $=\$ B \$ 7^{*} \$ D 22^{*}$ Relocation!\$F22*N22 \\
\hline 23 & $\mathrm{Pu}-238$ & $=$ Relocation!Y23 & $=\mathrm{C} 23 / \$ \mathrm{C} \$ 39$ & $=\$ B \$ 7^{*} \$ B \$ 3^{*} \$ B \$ 4^{*} \$ B \$ 5^{*} \$ B \$ 6^{*} D 23^{*}$ RelocationIH23*N23 & $=\$ B \$ 7^{*} \$ D 23^{*}$ Relocation!E23*N23 & $=\$ B \$ 7^{*} \$ D 23^{*}$ Relocation $1 \$ F 23^{*}$ N23 \\
\hline 24 & Pu-239 & $=$ Relocation!Y24 & $=\mathrm{C} 24 / \$ \mathrm{C} \$ 39$ & $=\$ B \$ 7^{*} \$ B \$ 3^{*} \$ B \$ 4^{*} \$ B \$ 5^{*} \$ B \$ 6^{*} D 24^{*}$ Relocation!H $24^{*} \mathrm{~N} 24$ & $=\$ B \$ 7^{*} \$ D 24^{*}$ Relocation!E24*N24 & $=\$ B \$ 7^{*} \$ D 24^{*}$ Relocation! $\$ F 24^{*} \mathrm{~N} 24$ \\
\hline 25 & Pu-239/241 & $=$ Relocation!Y25 & $=C 25 / \$ C \$ 39$ & & & \\
\hline 26 & Pu-240 & $=$ Relocation!Y26 & $=\mathrm{C} 26 / \$ \mathrm{C} \$ 39$ & $=\$ B \$ 7^{*} \$ B \$ 3^{*} \$ B \$ 4^{*} \$ B \$ 5^{*} \$ B \$ 6^{*} D 26^{*}$ Relocation!H26*N26 & $=\$ B \$ 7^{*} \$ D 26^{*}$ RelocationiE26*N26 & $=\$ B \$ 7 * \$ D 26 *$ Relocation! $\$ F 26 * N 26$ \\
\hline 27 & Pu-241 & $=$ Relocation!Y27 & $=\mathrm{C} 27 / \$ \mathrm{C} \$ 39$ & $=\$ B \$ 7^{*} \$ B \$ 3^{*} \$ B \$ 4^{*} \$ B \$ 5^{*} \$ B \$ 6^{*} D 27^{*}$ Relocation!H27*N27 & $=\$ B \$ 7^{*} \$ D 27^{*}$ Relocation!E27*N27 & $=\$ B \$ 7^{*} \$ D 27^{*}$ Relocation $1 \$ F 27^{*}$ N27 \\
\hline 28 & Pu-242 & $=$ Relocation!Y28 & $=\mathrm{C} 28 / \$ \mathrm{C} \$ 39$ & $=\$ B \$ 7^{*} \$ B \$ 3^{*} \$ B \$ 4^{*} \$ B \$ 5^{*} \$ B \$ 6^{*} D 28^{*}$ Relocation!H28*N28 & $=\$ B \$ 7^{*} \$ D 28^{*}$ Relocation!E28*N28 & $=\$ B \$ 7^{*} \$ \mathrm{D} 28^{*}$ Relocation! $\$ 28^{*} \mathrm{~N} 28$ \\
\hline 29 & Sr-90 & $=$ Relocation!Y29 & $=\mathrm{C} 29 / \$ \mathrm{C} \$ 39$ & $=\$ B \$ 7^{*} \$ B \$ 3^{*} \$ B \$ 4^{*} \$ B \$ 5^{*} \$ B \$ 6^{*} D 29^{*}$ Relocation!H29*N29 & $=\$ B \$ 7^{*} \$ D 29^{*}$ Relocation!E29*N29 & $=\$ B \$ 7^{*} \$ D 29^{*}$ Relocation $1 \$ F 29^{*}$ N29 \\
\hline 30 & Tc-99 & $=$ RelocationIY30 & $=\mathrm{C} 30 / \$ \mathrm{C} \$ 39$ & $=\$ B \$ 7^{*} \$ B \$ 3^{*} \$ B \$ 4^{*} \$ B \$ 5^{*} \$ B \$ 6^{*} D 30^{*}$ Relocation!H30*N30 & $=\$ B \$ 7 * \$ D 30^{*}$ Relocation!E30*N30 & $=\$ B \$ 7^{*} \$ D 30^{*}$ Relocation $\mid \$ F 30^{*} N 30$ \\
\hline 31 & Th-232 & $=$ Relocation!Y31 & $=C 31 / \$ C \$ 39$ & $=\$ B \$ 7^{*} \$ B \$ 3^{*} \$ B \$ 4^{*} \$ B \$ 5^{*} \$ B \$ 6^{*} D 31^{*}$ Relocation!H31*N31 & $=\$ B \$ 7^{*} \$ D 31^{*}$ Relocation!E31*N31 & $=\$ B \$ 7^{*} \$ D 31 *$ Relocation $1 \$ F 31 * N 31$ \\
\hline 32 & TI-204 & $=$ RelocationlY32 & $=\mathrm{C} 32 / \$ \mathrm{C} \$ 39$ & $=\$ B \$ 7^{*} \$ B \$ 3^{*} \$ B \$ 4^{*} \$ B \$ 5^{*} \$ B \$ 6^{*} \mathrm{D} 32^{*}$ Relocation!H32*N32 & $=\$ B \$ 7 * \$ D 32^{*}$ Relocation!E32*N32 & $=\$ B \$ 7^{*} \$ D 32^{*}$ Relocation $1 \$ F 32^{*}$ N32 \\
\hline 33 & U.235 & $=$ RelocationlY33 & $=C 33 / \$ C \$ 39$ & $=\$ B \$ 7^{*} \$ B \$ 3^{*} \$ B \$ 4^{*} \$ B \$ 5^{*} \$ B \$ 6^{*} D 33^{*}$ Relocation!H33*N33 & $=\$ B \$ 7^{*} \$ D 33^{*}$ Relocation!E33*N33 & $=\$ B \$ 7^{*} \$ D 33^{*}$ Relocation $! \$ F 33^{*} \mathrm{~N} 33$ \\
\hline 34 & U-238 & $=$ RelocationIY34 & $=\mathrm{C} 34 / \$ \mathrm{C} \$ 39$ & $=\$ B \$ 7^{*} \$ B \$ 3^{*} \$ B \$ 4^{*} \$ B \$ 5^{*} \$ B \$ 6^{*} D 34^{*}$ Relocation!H34*N34 & $=\$ B \$ 7^{*} \$ D 34^{*}$ Relocation!E34*N34 & $=\$ B \$ 7^{*} \$ D 34^{*}$ Relocation $1 \$ F 34^{*}$ N34 \\
\hline 35 & & & & & & \\
\hline 36 & Ba-137m & $=$ Relocation!Y36 & $=\mathrm{C} 36 / \$ C \$ 39$ & & & \\
\hline 37 & $Y-90$ & $=$ Relocation!Y37 & $=\mathrm{C} 37 / \$ \mathrm{C} \$ 39$ & & & \\
\hline 38 & & & & & & \\
\hline 39 & Total & $=$ SUM(C10:C38) & $=$ SUM $(D 10: D 38$, & $=S U M(F 10: F 38)$ & $=$ SUM(G10:G38) & $=$ SUM $(110: 138)$ \\
\hline 40 & & & & & & \\
\hline 41 & \multicolumn{2}{|l|}{ Dose in st } & & $=$ F39-'Relo_H 1 yr'IF39 & $=$ G39-'Relo_H 1yr'!G39 & $=\mid 39-{ }^{-}$Relo_H tyr'||39 \\
\hline
\end{tabular}




\begin{tabular}{|c|c|c|c|c|}
\hline & $\mathrm{J}$ & $\mathrm{K}$ & $\mathrm{L}$ & $\mathrm{N}$ \\
\hline 9 & BS Dose (mrem per uCl/m^2) & Halflife (years) & Lambda1 & Weathering Factor ( 2 years) \\
\hline 10 & $=\$ B \$ 7^{*} \$ B \$ 3^{*} \$ B \$ 4^{*} \$ B \$ 5^{*} \$ B \$ 6^{*} \$ D 10^{*}$ Relocation $\$ 110^{*} \mathrm{~N} 10$ & $=T_{-}$halflC2 & $=0.693 / \mathrm{K} 10$ & $=\left(0.63 /(\$ L 10+\$ K \$ 5)^{*}\left(1-\operatorname{EXP}\left(-(\$ L 10+\$ K \$ 5)^{*} \$ N \$ 6\right)\right)\right)+\left((0.37 /(\$ L 10+\$ K \$ 7))^{*}\left(1-\operatorname{EXP}\left(-(\$ L 10+\$ K \$ 7)^{*} \$ N \$ 6\right)\right)\right)$ \\
\hline 11 & $=\$ B \$ 7^{*} \$ B \$ 3^{*} \$ B \$ 4^{*} \$ B \$ 5^{*} \$ B \$ 6^{*} \$ D 11^{*}$ Relocation $1 \$ 11^{*} \mathrm{~N} 11$ & $=T$ half!C3 & $=0.693 / \mathrm{K} 11$ & $=\left(0.63 /(\$ L 11+\$ K \$ 5)^{*}\left(1-\operatorname{EXP}\left(-(\$ L 11+\$ K \$ 5)^{*} \$ N \$ 6\right)\right)\right)+\left((0.37 /(\$ L 11+\$ K \$ 7))^{*}\left(1-\operatorname{EXP}\left(-(\$ L 11+\$ K \$ 7)^{*} \$ N \$ 6\right)\right)\right)$ \\
\hline 12 & $=\$ B \$ 7^{*} \$ B \$ 3^{*} \$ B \$ 4^{*} \$ B \$ 5^{*} \$ B \$ 6^{*} \$ D 12^{*}$ Relocation $\$ 12^{*} N 12$ & $=T$ halfic4 & $=0.693 / \mathrm{K} 12$ & $=\left(0.63 /(\$ L 12+\$ K \$ 5)^{*}\left(1-\operatorname{EXP}\left(-(\$ L 12+\$ K \$ 5)^{*} \$ N \$ 6\right)\right)\right)+\left((0.37 /(\$ L 12+\$ K \$ 7))^{*}\left(1-\operatorname{EXP}\left(-(\$ L 12+\$ K \$ 7)^{*} \$ N \$ 6\right)\right)\right)$ \\
\hline 13 & $=\$ B \$ 7^{*} \$ B \$ 3^{*} \$ B \$ 4^{*} \$ B \$ 5^{*} \$ B \$ 6^{*} \$ D 13^{*}$ Relocation! $\$ 13^{*} N 13$ & $=T \_$halff 55 & $=0.693 / \mathrm{K} 13$ & $=\left(0.63 /(\$ L 13+\$ K \$ 5)^{*}\left(1-\operatorname{EXP}\left(-(\$ L 13+\$ K \$ 5)^{*} \$ N \$ 6\right)\right)\right)+\left((0.37 /(\$ L 13+\$ K \$ 7))^{*}\left(1-\operatorname{EXP}\left(-(\$ L 13+\$ K \$ 7)^{*} \$ N \$ 6\right)\right)\right)$ \\
\hline 14 & $=\$ B \$ 7^{*} \$ B \$ 3^{*} \$ B \$ 4^{*} \$ B \$ 5^{*} \$ B \$ 6^{*} \$ D 14^{*}$ Relocation! $\$ 114^{*} \mathrm{~N} 14$ & $=T_{-}$half!C6 & $=0.693 / \mathrm{k} 14$ & $=\left(0.63 /(\$ L 14+\$ K \$ 5)^{*}\left(1-\operatorname{EXP}\left(-(\$ L 14+\$ K \$ 5)^{*} \$ N \$ 6\right)\right)\right)+\left((0.37 /(\$ L 14+\$ K \$ 7))^{*}\left(1-\operatorname{EXP}\left(-(\$ L 14+\$ K \$ 7)^{*} \$ N \$ 6\right)\right)\right)$ \\
\hline 15 & ]$=\$ B \$ 7 * \$ B \$ 3^{*} \$ B \$ 4^{*} \$ B \$ 5^{*} \$ B \$ 6 * \$ D 15^{*}$ Relocation! $\$ J 15^{*} \mathrm{~N} 15$ & $=T$ _half $! C 7$. & $=0.693 / \mathrm{K} 15$ & $=\left(0.63 /(\$ L 15+\$ K \$ 5)^{*}\left(1-\operatorname{EXP}\left(-(\$ L 15+\$ K \$ 5)^{*} \$ N \$ 6\right)\right)\right)+\left((0.37 /(\$ L 15+\$ K \$ 7))^{*}\left(1-\operatorname{EXP}\left(-(\$ L 15+\$ K \$ 7)^{*} \$ N \$ 6\right)\right)\right)$ \\
\hline 16 & $=\$ B \$ 7^{*} \$ B \$ 3^{*} \$ B \$ 4^{*} \$ B \$ 5^{*} \$ B \$ 6^{*} \$ D 16^{*}$ Relocation $1 \$ 16^{*} N 16$ & $=T$ half $1 \mathrm{C} 8$ & $=0.693 / \mathrm{K} 16$ & $=\left(0.63 /(\$ L 16+\$ K \$ 5)^{*}\left(1-\operatorname{EXP}\left(-(\$ L 16+\$ K \$ 5)^{*} \$ N \$ 6\right)\right)\right)+\left((0.37 /(\$ L 16+\$ K \$ 7))^{*}\left(1-\operatorname{EXP}\left(-(\$ L 16+\$ K \$ 7)^{*} \$ N \$ 6\right)\right)\right)$ \\
\hline 17 & $=\$ B \$ 7^{*} \$ B \$ 3^{*} \$ B \$ 4^{*} \$ B \$ 5^{*} \$ B \$ 6^{*} \$ D 17^{*}$ Relocation $1 \$ 17^{*} N 17$ & $=T_{-}$halfiC9 & $=0.693 / \mathrm{K} 17$ & $=\left(0.63 /(\$ L 17+\$ K \$ 5)^{*}\left(1-\operatorname{EXP}\left(-(\$ L 17+\$ K \$ 5)^{*} \$ N \$ 6\right)\right)\right)+\left((0.37 /(\$ L 17+\$ K \$ 7))^{*}\left(1-\operatorname{EXP}\left(-(\$ L 17+\$ K \$ 7)^{*} \$ N \$ 6\right)\right)\right)$ \\
\hline 18 & $=\$ B \$ 7^{*} \$ B \$ 3^{*} \$ B \$ 4^{*} \$ B \$ 5^{*} \$ B \$ 6^{*} \$ D 18^{*}$ Relocation! $\$ 18^{*} N 18$ & $=T$ _halfic10 & $=0.693 / \mathrm{K} 18$ & $=\left(0.63 /(\$ L 18+\$ K \$ 5)^{*}\left(1-\operatorname{EXP}\left(-(\$ L 18+\$ K \$ 5)^{*} \$ N \$ 6\right)\right)\right)+\left((0.37 /(\$ L 18+\$ K \$ 7))^{*}\left(1-\operatorname{EXP}\left(-(\$ L 18+\$ K \$ 7)^{*} \$ N \$ 6\right)\right)\right)$ \\
\hline 19 & $=\$ B \$ 7^{*} \$ B \$ 3^{*} \$ B \$ 4^{*} \$ B \$ 5^{*} \$ B \$ 6^{*} \$ D 19^{*}$ Relocation! $\$ 119^{*} \mathrm{~N} 19$ & $=T$ halfic11 & $=0.693 / \mathrm{K} 19$ & $=\left(0.63 /(\$ L 19+\$ K \$ 5)^{*}\left(1-\operatorname{EXP}\left(-(\$ L 19+\$ K \$ 5)^{*} \$ N \$ 6\right)\right)\right)+\left((0.37 /(\$ L 19+\$ K \$ 7))^{*}\left(1-\operatorname{EXP}\left(-(\$ L 19+\$ K \$ 7)^{*} \$ N \$ 6\right)\right)\right)$ \\
\hline 20 & $=\$ B \$ 7^{*} \$ B \$ 3^{*} \$ B \$ 4^{*} \$ B \$ 5^{*} \$ B \$ 6^{*} \$ D 20^{*}$ Relocation $\$ J 20^{*} \mathrm{~N} 20$ & $=T$ _halfic12 & $=0.693 / \mathrm{K} 20$ & $=\left(0.63 /(\$ L 20+\$ K \$ 5)^{*}\left(1-\operatorname{EXP}\left(-(\$ L 20+\$ K \$ 5)^{*} \$ N \$ 6\right)\right)\right)+\left((0.37 /(\$ L 20+\$ K \$ 7))^{*}\left(1-\operatorname{EXP}\left(-(\$ L 20+\$ K \$ 7)^{*} \$ N \$ 6\right)\right)\right)$ \\
\hline 21 & $=\$ B \$ 7^{*} \$ B \$ 3^{*} \$ B \$ 4^{*} \$ B \$ 5^{*} \$ B \$ 6^{*} \$ D 21^{*}$ Relocation! $\$ J 21 * N 21$ & $=T_{-}$half!C 13 & $=0.693 / \mathrm{K} 21$ & $=\left(0.63 /(\$ L 21+\$ K \$ 5)^{*}\left(1-\operatorname{EXP}\left(-(\$ L 21+\$ K \$ 5)^{*} \$ N \$ 6\right)\right)\right)+\left((0.37 /(\$ L 21+\$ K \$ 7))^{*}\left(1-\operatorname{EXP}\left(-(\$ L 21+\$ K \$ 7)^{*} \$ N \$ 6\right)\right)\right)$ \\
\hline 22 & $=\$ B \$ 7^{*} \$ B \$ 3^{*} \$ B \$ 4^{*} \$ B \$ 5^{*} \$ B \$ 6^{*} \$ D 22^{*}$ Relocation! $\$ J 22^{*} \mathrm{~N} 22$ & $=T$ _halfIC14 & $=0.693 / \mathrm{K} 22$ & $=\left(0.63 /(\$ L 22+\$ K \$ 5)^{*}\left(1-\operatorname{EXP}\left(-(\$ L 22+\$ K \$ 5)^{*} \$ N \$ 6\right)\right)\right)+\left((0.37 /(\$ L 22+\$ K \$ 7))^{*}\left(1-\operatorname{EXP}\left(-(\$ L 22+\$ K \$ 7)^{*} \$ N \$ 6\right)\right)\right)$ \\
\hline 23. & $=\$ B \$ 7^{*} \$ B \$ 3^{*} \$ B \$ 4^{*} \$ B \$ 5^{*} \$ B \$ 6^{*} \$ D 23^{*}$ Relocation! $\$ J 23^{*} \mathrm{~N} 23$ & $=T_{\text {__half } ! \mathrm{C} 15}$ & $=0.693 / \mathrm{K} 23$ & $=\left(0.63 /(\$ L 23+\$ K \$ 5)^{*}\left(1-\operatorname{EXP}\left(-(\$ L 23+\$ K \$ 5)^{*} \$ N \$ 6\right)\right)\right)+\left((0.37 /(\$ L 23+\$ K \$ 7))^{*}\left(1-\operatorname{EXP}\left(-(\$ L 23+\$ K \$ 7)^{*} \$ N \$ 6\right)\right)\right)$ \\
\hline$\frac{24}{25}$ & $=\$ B \$ 7^{*} \$ B \$ 3^{*} \$ B \$ 4^{*} \$ B \$ 5^{*} \$ B \$ 6^{*} \$ D 24^{*}$ Relocation! $\$ J 24^{*} N 24$ & $=T \_$half!C16 & $=0.693 / \mathrm{K} 24$ & $=\left(0.63 /(\$ L 24+\$ K \$ 5)^{*}\left(1-\operatorname{EXP}\left(-(\$ L 24+\$ K \$ 5)^{*} \$ N \$ 6\right)\right)\right)+\left((0.37 /(\$ L 24+\$ K \$ 7))^{*}\left(1-\operatorname{EXP}\left(-(\$ L 24+\$ K \$ 7)^{*} \$ N \$ 6\right)\right)\right)$ \\
\hline 26 & $=\$ B \$ 7^{*} \$ B \$ 3^{*} \$ B \$ 4^{*} \$ B \$ 5^{*} \$ B \$ 6^{*} \$ D 26^{*}$ Relocation! $\$ J 26^{*} \mathrm{~N} 26$ & $=T_{\text {__halfiC18 }}$ & $=0.693 / \mathrm{K} 26$ & $=\left(0.63 /(\$ \mathrm{~L} 26+\$ K \$ 5)^{*}\left(1-\operatorname{EXP}\left(-(\$ L .26+\$ K \$ 5)^{*} \$ N \$ 6\right)\right)\right)+\left((0.37 /(\$ L 26+\$ K \$ 7))^{*}\left(1-\operatorname{EXP}\left(-(\$ L 26+\$ K \$ 7)^{*} \$ N \$ 6\right)\right)\right)$ \\
\hline 27 & $=\$ B \$ 7^{*} \$ B \$ 3^{*} \$ B \$ 4^{*} \$ B \$ 5^{*} \$ B \$ 6^{*} \$ D 27^{*}$ Relocation! $\$ J 27^{*} \mathrm{~N} 27$ & $=T$ Thalfic19 & $=0.693 / \mathrm{K} 27$ & $=\left(0.63 /(\$ L 27+\$ K \$ 5)^{*}\left(1-\operatorname{EXP}\left(-(\$ L 27+\$ K \$ 5)^{*} \$ N \$ 6\right)\right)\right)+\left((0,37 /(\$ L 27+\$ K \$ 7))^{*}\left(1-\operatorname{EXP}\left(-(\$ L 27+\$ K \$ 7)^{*} \$ N \$ 6\right)\right)\right)$ \\
\hline 28 & $=\$ B \$ 7^{*} \$ B \$ 3^{*} \$ B \$ 4^{*} \$ B \$ 5^{*} \$ B \$ 6^{*} \$ D 28^{*}$ Relocation $1 \$ J 28^{*} \mathrm{~N} 28$ & $=T_{-}$half!C20 & $=0.693 / \mathrm{K} 28$ & $=\left(0.63 /(\$ L 28+\$ K \$ 5)^{*}\left(1-\operatorname{EXP}\left(-(\$ L 28+\$ K \$ 5)^{*} \$ N \$ 6\right)\right)\right)+\left((0.37 /(\$ L 28+\$ K \$ 7))^{*}\left(1-\operatorname{EXP}\left(-(\$ L 28+\$ K \$ 7)^{*} \$ N \$ 6\right)\right)\right)$ \\
\hline 29 & $=\$ B \$ 7^{*} \$ B \$ 3^{*} \$ B \$ 4^{*} \$ B \$ 5^{*} \$ B \$ 6^{*} \$ D 29^{*}$ Relocation $1 \$ J 29^{*} N 29$ & $=T_{-}$halfic21 & $=0.693 / \mathrm{K} 29$ & $=\left(0.63 /(\$ L 29+\$ K \$ 5)^{*}\left(1-\operatorname{EXP}\left(-(\$ L 29+\$ K \$ 5)^{*} \$ N \$ 6\right)\right)\right)+\left((0.37 /(\$ L 29+\$ K \$ 7))^{*}\left(1-\operatorname{EXP}\left(-(\$ L 29+\$ K \$ 7)^{*} \$ N \$ 6\right)\right)\right)$ \\
\hline 30 & $=\$ B \$ 7^{*} \$ B \$ 3^{*} \$ B \$ 4^{*} \$ B \$ 5^{*} \$ B \$ 6^{*} \$ D 30^{*}$ Relocation $1 \$ \sqrt{3} 0^{*} N 30$ & $=T_{\text {_halfic22 }}$ & $=0.693 / \mathrm{K} 30$ & $=\left(0.63 /(\$ L 30+\$ K \$ 5)^{*}\left(1-\operatorname{EXP}\left\{(\$ L 30+\$ K \$ 5)^{*} \$ N \$ 6\right)\right)\right)+\left((0.37 /(\$ L 30+\$ K \$ 7))^{*}\left(1-\operatorname{EXP}\left(-(\$ L 30+\$ K \$ 7)^{*} \$ N \$ 6\right)\right)\right)$ \\
\hline 31 & $=\$ B \$ 7^{*} \$ B \$ 3^{*} \$ B \$ 4^{*} \$ B \$ 5^{*} \$ B \$ 6^{*} \$ D 31^{*}$ Relocation! $\$ 331^{*} N 31$ & $=T_{\text {__halfIC23 }}$ & $=0.693 / \mathrm{K} 31$ & $=\left(0.63 /(\$ L 31+\$ K \$ 5)^{*}\left(1-\operatorname{EXP}\left(-(\$ L 31+\$ K \$ 5)^{*} \$ N \$ 6\right)\right)\right)+\left((0.37 /(\$ L 31+\$ K \$ 7))^{*}\left(1-\operatorname{EXP}\left(-(\$ L 31+\$ K \$ 7)^{*} \$ N \$ 6\right)\right)\right)$ \\
\hline 32 & $=\$ B \$ 7^{*} \$ B \$ 3^{*} \$ B \$ 4^{*} \$ B \$ 5^{*} \$ B \$ 6^{*} \$ D 32^{*}$ Relocation! $\$ J 32^{*} \mathrm{~N} 32$ & $=T_{\text {_halflC24 }}$ & $=0.693 / \mathrm{K} 32$ & $=\left(0.63 /(\$ L 32+\$ K \$ 5)^{*}\left(1-\operatorname{EXP}\left(-(\$ L 32+\$ K \$ 5)^{*} \$ N \$ 6\right)\right)\right)+\left((0.37 /(\$ L 32+\$ K \$ 7))^{*}\left(1-\operatorname{EXP}\left(-(\$ L 32+\$ K \$ 7)^{*} \$ N \$ 6\right)\right)\right)$ \\
\hline 33 & $=\$ B \$ 7^{*} \$ B \$ 3^{*} \$ B \$ 4^{*} \$ B \$ 5^{*} \$ B \$ 6^{*} \$ D 33^{*}$ Relocation! $\$ 333^{*} N 33$ & $=T_{\text {T_half! }} \mathrm{C} 25$ & $=0.693 / \mathrm{K} 33$ & $=\left(0.63 /(\$ L 33+\$ K \$ 5)^{*}\left(1-\operatorname{EXP}\left(-(\$ L 33+\$ K \$ 5)^{*} \$ N \$ 6\right)\right)\right)+\left((0.37 /(\$ L 33+\$ K \$ 7))^{*}\left(1-\operatorname{EXP}\left(-(\$ L 33+\$ K \$ 7)^{*} \$ N \$ 6\right)\right)\right)$ \\
\hline$\frac{34}{35}$ & $=\$ B \$ 7^{*} \$ B \$ 3^{*} \$ B \$ 4^{*} \$ B \$ 5^{*} \$ B \$ 6^{*} \$ D 34^{*}$ Relocation! $\$ J 34^{*} N 34$ & $=T \_$half!C26 & $=0.693 / \mathrm{K} 34$ & $=\left(0.63 /(\$ L 34+\$ K \$ 5)^{*}\left(1-\operatorname{EXP}\left(-(\$ L 34+\$ K \$ 5)^{*} \$ N \$ 6\right)\right)\right)+\left((0.37 /(\$ L 34+\$ K \$ 7))^{*}\left(1-\operatorname{EXP}\left(-(\$ L 34+\$ K \$ 7)^{*} \$ N \$ 6\right)\right)\right)$ \\
\hline 36 & & $=T_{\text {_half } ! C 28}$ & $=0.693 / \mathrm{K} 36$ & $=\left(0.63 /(\$ L 36+\$ K \$ 5)^{*}\left(1-\operatorname{EXP}\left(-(\$ L 36+\$ K \$ 5)^{*} \$ N \$ 6\right)\right)\right)+\left((0.37 /(\$ L 36+\$ K \$ 7))^{*}\left(1-\operatorname{EXP}\left(-(\$ L 36+\$ K \$ 7)^{*} \$ N \$ 6\right)\right)\right)$ \\
\hline 37 & & $=T_{\text {_half } \mid \mathrm{C} 29}$ & $=0.693 / \mathrm{K} 37$ & $=\left(0.63 /(\$ L 37+\$ K \$ 5)^{*}\left(1-\operatorname{EXP}\left(-(\$ L 37+\$ K \$ 5)^{*} \$ N \$ 6\right)\right)\right)+\left((0.37 /(\$ L 37+\$ K \$ 7))^{*}\left(1-\operatorname{EXP}\left(-(\$ L 37+\$ K \$ 7)^{*} \$ N \$ 6\right)\right)\right)$ \\
\hline 38 & & & & \\
\hline 39 & $=S U M(J 10: J 38)$ & & & \\
\hline 40 & & & & \\
\hline$\frac{41}{42}$ & $=\sqrt{3} 39$ & & & \\
\hline
\end{tabular}




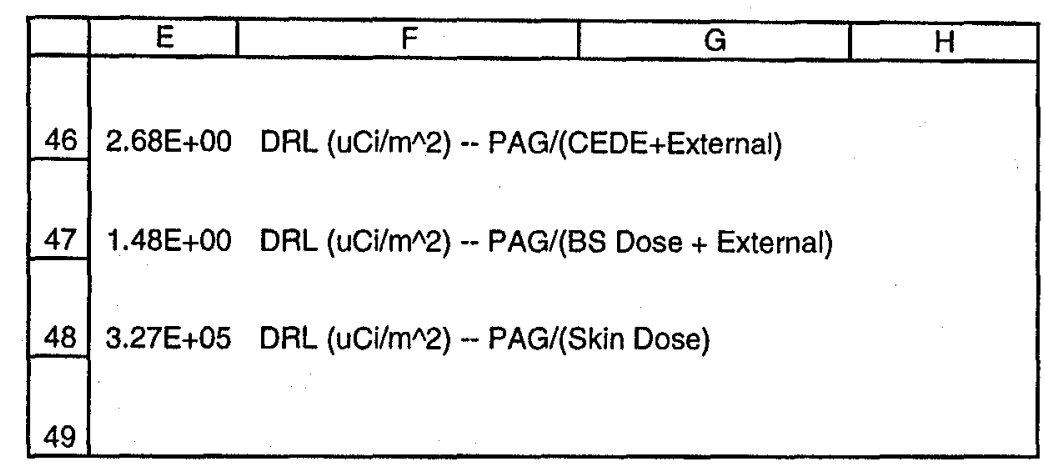




\begin{tabular}{|c|c|c|c|}
\hline & $E$ & $\mathbf{F}$ & $\bar{G}$ \\
\hline 46 & $=F 3 /(F 41+G 41)$ & DRL $\left(u C l / m^{\wedge} 2\right)$ - PAG/(CEDE+External) & \\
\hline 47 & $=F 4 /(J 41+G 41)$ & DRL (UCi/m^2) -- PAG/(BS Dose + External) & \\
\hline 48 & $=F 5 / 141$ & DRL (uCi/m^2) -- PAG/(Skin Dose) & \\
\hline 49 & & & \\
\hline
\end{tabular}




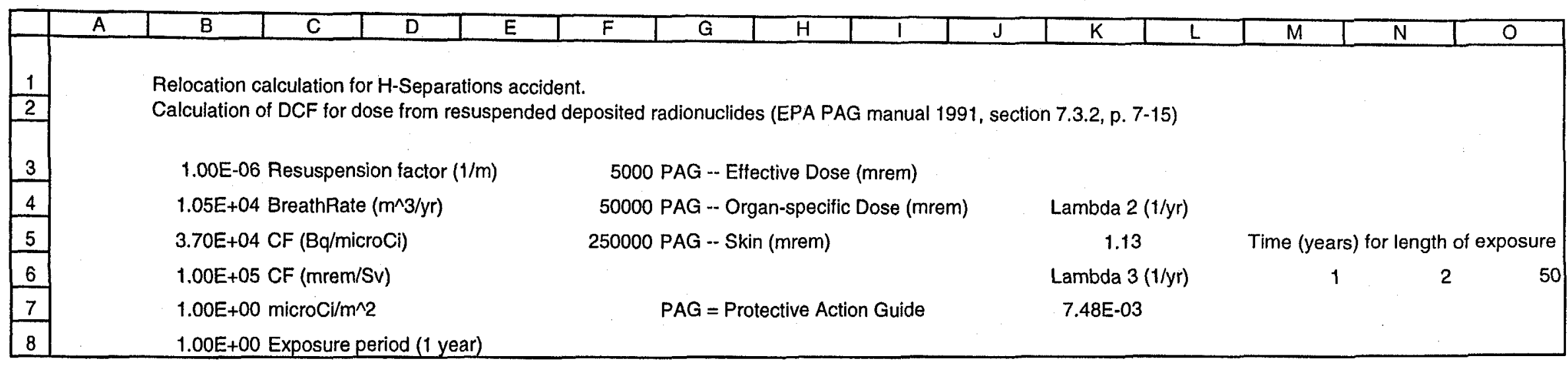




\begin{tabular}{|c|c|c|c|c|c|c|c|c|c|c|c|c|}
\hline & $\mathrm{B}$ & $\mathrm{C}$ & $\mathrm{D}$ & $E$ & $\mathrm{~F}$ & G & $\mathrm{H}$ & 1 & $J$ & $\mathrm{~K}$ & $L$ & 0 \\
\hline 9 & Nuclide & $\begin{array}{l}\text { Release } \\
\text { (Ci) }\end{array}$ & $\begin{array}{l}\text { Activity } \\
\text { fraction }\end{array}$ & & $\begin{array}{l}\text { CEDE } \\
\text { (mrem per } \\
\mathrm{UCl} / \mathrm{m}^{\wedge} 2 \text { ) }\end{array}$ & $\begin{array}{l}\text { External } \\
\text { EDE } \\
\text { (mrem per } \\
\mathrm{uCi} / \mathrm{m}^{\wedge} 2 \text { ) }\end{array}$ & & $\begin{array}{l}\text { Skin Dose } \\
\text { (mrem per } \\
\mathrm{uCl} / \mathrm{m}^{\wedge} 2 \text { ) }\end{array}$ & $\begin{array}{l}\text { BS Dose } \\
\text { (mrem per } \\
\text { UCl/m^2) }\end{array}$ & $\begin{array}{l}\text { Halflife } \\
\text { (years) }\end{array}$ & Lambda1 & $\begin{array}{l}\text { Weathering } \\
\text { Factor (50 } \\
\text { years) }\end{array}$ \\
\hline 10 & Am-241 & $6.57 \mathrm{E}-01$ & 1.87E-02 & & $1.35 E+03$ & 8.64E-01 & & $1.85 E+00$ & $2.44 E+04$ & 458 & 0.001513 & $1.546 \mathrm{E}+01$ \\
\hline 11 & Am-243 & $0.00 E+00$ & $0.00 E+00$ & & $0.00 E+00$ & $0.00 E+00$ & & $0.00 \mathrm{E}+00$ & $0.00 E+00$ & 7950 & 8.72E-05 & $1.596 E+01$ \\
\hline 12 & $\mathrm{Ba}-133$ & $0.00 \mathrm{E}+00$ & $0.00 E+00$ & & $0.00 E+00$ & $0.00 E+00$ & & $0.00 E+00$ & $0.00 \mathrm{E}+00$ & 10.5 & 0.066 & $5.434 \mathrm{E}+00$ \\
\hline 13 & $\mathrm{Ce}-144$ & $0.00 E+00$ & $0.00 \mathrm{E}+00$ & & $0.00 E+00$ & $0.00 E+00$ & & $0.00 E+00$ & $0.00 E+00$ & 0.778082 & 0.890651 & 7.237E-01 \\
\hline 14 & Cf-249 & $0.00 E+00$ & $0.00 E+00$ & & $0.00 E+00$ & $0.00 E+00$ & & $0.00 \mathrm{E}+00$ & $0.00 E+00$ & 360 & 0.001925 & $1.532 E+01$ \\
\hline 15 & $C f-252$ & $0.00 E+00$ & $0.00 \mathrm{E}+00$ & & $0.00 E+00$ & $0.00 E+00$ & & $0.00 E+00$ & $0.00 \mathrm{E}+00$ & 2.646 & 0.261905 & $1.826 \mathrm{E}+00$ \\
\hline 16 & $\mathrm{Cm}-244$ & $0.00 E+00$ & $0.00 \mathrm{E}+00$ & & $0.00 E+00$ & $0.00 \mathrm{E}+00$ & & $0.00 E+00$ & $0.00 E+00$ & 17.6 & 0.039375 & $7.677 E+00$ \\
\hline 17 & $\mathrm{Cm}-246$ & $0.00 E+00$ & $0.00 E+00$ & & $0.00 E+00$ & $0.00 E+00$ & & $0.00 E+00$ & $0.00 \mathrm{E}+00$ & 5500 & 0.000126 & $1.595 \mathrm{E}+01$ \\
\hline 18 & Co-60 & $0.00 \mathrm{E}+00$ & $0.00 E+00$ & & $0.00 \mathrm{E}+00$ & $0.00 \mathrm{E}+00$ & & $0.00 E+00$ & $0.00 \mathrm{E}+00$ & 5.26 & 0.131749 & $3.154 E+00$ \\
\hline 19 & Cs-137 & $0.00 E+00$ & $0.00 \mathrm{E}+00$ & & $0.00 E+00$ & $0.00 E+00$ & & $0.00 E+00$ & $0.00 E+00$ & 30 & 0.0231 & $1.002 E+01$ \\
\hline 20 & $\mathrm{H}-3$ & $0.00 E+00$ & $0.00 E+00$ & & $0.00 E+00$ & $0.00 E+00$ & & $0.00 E+00$ & $0.00 E+00$ & 12.3 & 0.056341 & $6.090 \mathrm{E}+00$ \\
\hline 21 & $\mathrm{~Np}-237$ & $0.00 \mathrm{E}+00$ & $0.00 E+00$ & & $0.00 E+00$ & $0.00 E+00$ & & $0.00 E+00$ & $0.00 \mathrm{E}+00$ & 2140000 & $3.24 E-07$ & $1.599 E+01$ \\
\hline 22 & $\mathrm{Pm}-147$ & $0.00 E+00$ & $0.00 E+00$ & & $0.00 E+00$ & $0.00 \mathrm{E}+00$ & & $0.00 E+00$ & $0.00 E+00$ & 2.62 & 0.264504 & $1.812 E+00$ \\
\hline 23 & Pu-238 & $1.62 E+00$ & 4.61E-02 & & $2.55 E+03$ & 5.31E-02 & & 4.44E-01 & 4.57E+04 & 86.4 & 0.008021 & $1.343 E+01$ \\
\hline 24 & Pu-239 & $4.23 E-03$ & 1.20E-04 & & $8.67 E+00$ & 7.27E-05 & & $5.39 E-04$ & $1.58 E+02$ & 24390 & $2.84 \mathrm{E}-05$ & $1.598 E+01$ \\
\hline 25 & $\mathrm{Pu}-239 / 241$ & $0.00 E+00$ & $0.00 \mathrm{E}+00$ & & & & & & & & & \\
\hline 26 & $P u-240$ & $2.43 \mathrm{E}-01$ & $6.92 E-03$ & & 4.97E+02 & $9.05 E-03$ & & 7.54E-02 & $9.04 \mathrm{E}+03$ & 6580 & 0.000105 & $1.595 \mathrm{E}+01$ \\
\hline 27 & Pu-241 & $3.26 \mathrm{E}+01$ & $9.28 E-01$ & & $5.14 E+02$ & $0.00 \mathrm{E}+00$ & & $0.00 E+00$ & $9.68 E+03$ & 13.2 & 0.0525 & $6.394 E+00$ \\
\hline 28 & $P u-242$ & $1.54 \mathrm{E}-02$ & 4.38E-04 & & $3.02 E+01$ & 4.78E-04 & & 3.97E-03 & $5.47 E+02$ & 379000 & 1.83E-06 & $1.599 \mathrm{E}+01$ \\
\hline 29 & Sr-90 & $0.00 E+00$ & $0.00 E+00$ & & $0.00 E+00$ & $0.00 E+00$ & & $0.00 \mathrm{E}+00$ & $0.00 \mathrm{E}+00$ & 28.1 & 0.024662 & $9.749 E+00$ \\
\hline 30 & Tc-99 & $0.00 E+00$ & $0.00 E+00$ & & $0.00 E+00$ & $0.00 E+00$ & & $0.00 E+00$ & $0.00 E+00$ & 212000 & 3.27E-06 & $1.599 \mathrm{E}+01$ \\
\hline 31 & Th-232 & $0.00 E+00$ & $0.00 \mathrm{E}+00$ & & $0.00 \mathrm{E}+00$ & $0.00 E+00$ & & $0.00 E+00$ & $0.00 \mathrm{E}+00$ & $1.4 \mathrm{E}+10$ & 4.95E-11 & $1.599 \mathrm{E}+01$ \\
\hline 32 & $\mathrm{Tl}-204$ & $0.00 \mathrm{E}+00$ & $0.00 E+00$ & & $0.00 \mathrm{E}+00$ & $0.00 E+00$ & & $0.00 E+00$ & $0.00 E+00$ & 3.8 & 0.182368 & $2.429 E+00$ \\
\hline 33 & U-235 & $0.00 \mathrm{E}+00$ & $0.00 \mathrm{E}+00$ & & $0.00 E+00$ & $0.00 \mathrm{E}+00$ & & $0.00 E+00$ & $0.00 E+00$ & $7.1 E+08$ & $9.76 \mathrm{E}-10$ & $1.599 E+01$ \\
\hline 34 & U-238 & $0.00 E+00$ & $0.00 E+00$ & & $0.00 E+00$ & $0.00 E+00$ & & $0.00 E+00$ & $0.00 E+00$ & $4.51 E+09$ & $1.54 \mathrm{E}-10$ & $1.599 \mathrm{E}+01$ \\
\hline 35 & & & & & & & & & & & & \\
\hline 36 & Ba-137m & $0.00 \mathrm{E}+00$ & $0.00 E+00$ & & & & & & & 4.86E-06 & 142727.6 & 7.006E-06 \\
\hline$\frac{37}{38}$ & $Y-90$ & $0.00 E+00$ & $0.00 \mathrm{E}+00$ & & & & & & & 0.007317 & 94.7064 & $1.048 E-02$ \\
\hline$\frac{39}{40}$ & Total & $3.51 E+01$ & $1.00 E+00$ & & $4.95 E+03$ & $9.27 E-01$ & & $2.37 E+00$ & $8.95 E+04$ & & & \\
\hline
\end{tabular}




\begin{tabular}{|c|c|c|c|c|c|c|}
\hline & B & C & $\mathrm{D}$ & F & G & 1 \\
\hline 9 & Nuclide & Release (Ci) & Activity fraction & CEDE (mrem per uCl/m^2) & External EDE (mrem per uCi/m^2) & Skin Dose (mrem per uCi/m^2) \\
\hline 10 & $A m-241$ & $=$ Relocation!Y10 & $=\mathrm{C} 10 / \$ \mathrm{C} \$ 39$ & $=\$ B \$ 7^{\star} \$ B \$ 3^{*} \$ B \$ 4^{*} \$ B \$ 5^{*} \$ B \$ 6^{*} D 10^{*}$ Relocation!H10*010 & $=\$ B \$ 7^{*} \$ D 10^{*}$ Relocation!E $10^{*} 010$ & $=\$ B \$ 7^{*} \$ D 10^{*}$ Relocation $\$$ F $10^{*} 010$ \\
\hline 11 & Am-243 & $=$ Relocation!Y11 & $=\mathrm{C} 11 / \$ \mathrm{C} \$ 39$ & $=\$ B \$ 7^{*} \$ B \$ 3^{*} \$ B \$ 4^{*} \$ B \$ 5^{*} \$ B \$ 6^{*} D 11^{*}$ Relocation!H1 $1^{*} \mathrm{O} 11$ & $=\$ B \$ 7^{*} \$ D 11^{*}$ Relocation $\mid E 11^{*} 011$ & $=\$ B \$ 7^{*} \$ D 11^{*}$ Relocation! $\$ F 11^{*} 011$ \\
\hline 12 & Ba-133 & $=$ RelocationlY12 & $=C 12 / \$ C \$ 39$ & $=\$ B \$ 7^{*} \$ B \$ 3^{*} \$ B \$ 4^{*} \$ B \$ 5^{*} \$ B \$ 6^{*} D 12^{*}$ Relocation!H12* 012 & $=\$ B \$ 7^{*} \$ D 12^{*}$ Relocation!E12*012 & $=\$ \mathrm{~B} \$ 7^{*} \$ D 12^{*}$ Relocation! $\$ F 12^{*} \mathrm{O} 12$ \\
\hline 13 & Ce-144 & $=$ Relocation!Y13 & $=C 13 / \$ C \$ 39$ & $=\$ B \$ 7^{*} \$ B \$ 3^{*} \$ B \$ 4^{*} \$ B \$ 5^{*} \$ B \$ 6^{*} D 13^{*}$ Relocation!H13*013 & $=\$ B \$ 7^{*} \$ D 13^{*}$ Relocation!E13*013 & $=\$ B \$ 7^{*} \$ D 13^{*}$ Relocation! $\$ F 13^{*} \mathrm{O} 13$ \\
\hline 14 & Cf-249 & $=$ Relocation!Y14 & $=C 14 / \$ C \$ 39$ & $=\$ B \$ 7^{*} \$ B \$ 3^{*} \$ B \$ 4^{*} \$ B \$ 5^{*} \$ B \$ 6^{*} D 14^{*}$ RelocationlH14*014 & $=\$ B \$ 7^{*}$ \$D $14^{*}$ Relocation!E14*014 & $=\$ B \$ 7^{*} \$ D 14^{*}$ Relocation! $\$ F 14^{*} \mathrm{O} 14$ \\
\hline 15 & C1-252 & $=$ Relocation!Y15 & $=C 15 / \$ C \$ 39$ & $=\$ B \$ 7^{*} \$ B \$ 3^{*} \$ B \$ 4^{*} \$ B \$ 5^{*} \$ B \$ 6^{*} D 15^{*}$ Relocation!H15*015 & $=\$ B \$ 7^{*} \$ D 15^{*}$ Relocation!E15*O15 & $=\$ B \$ 7^{*} \$ D 15^{*}$ Relocation! $\$ F 15^{*} \mathrm{O} 15$ \\
\hline 16 & $\mathrm{Cm}-244$ & $=$ Relocation!Y16 & $=\mathrm{C} 16 / \$ \mathrm{C} \$ 39$ & $=\$ B \$ 7^{*} \$ B \$ 3^{*} \$ B \$ 4^{*} \$ B \$ 5^{*} \$ B \$ 6^{*} D 16^{*}$ Relocation!H16*016 & $=\$ B \$ 7^{*} \$ D 16^{*}$ Relocation!E16*016 & $=\$ B \$ 7^{*} \$ D 16^{*}$ Relocation! $\$ F 16^{*} 016$ \\
\hline 17 & $\mathrm{Cm}-246$ & $=$ Relocation!Y17 & $=C 17 / \$ C \$ 39$ & $=\$ B \$ 7^{*} \$ B \$ 3^{*} \$ B \$ 4^{*} \$ B \$ 5^{*} \$ B \$ 6^{*} D 17^{*}$ Relocation $1 \mathrm{H} 17^{*} \mathrm{O} 17$ & $=\$ B \$ 7^{*} \$ D 17^{*}$ Relocation!E $17^{*} 017$ & $=\$ B \$ 7^{*} \$ D 17^{*}$ Relocation! $\$ F 17^{*} 017$ \\
\hline 18 & Co-60 & $=$ Relocation!Y18 & $=C 18 / \$ C \$ 39$ & $=\$ B \$ 7^{*} \$ \mathrm{~B} \$ 3^{*} \$ \mathrm{~B} \$ 4^{*} \$ \mathrm{~B} \$ 5^{*} \$ \mathrm{~B} \$ 6^{*} \mathrm{D} 18^{*}$ Relocation!H18*018 & $=\$ B \$ 7^{*} \$ D 18^{*}$ Relocation!E $18^{*} 018$ & $=\$ B \$ 7^{*} \$ D 18^{*}$ Relocation! $\$ F 18^{*} 018$ \\
\hline 19 & Cs-137 & $=$ RelocationIY19 & $=C 19 / \$ C \$ 39$ & $=\$ B \$ 7^{*} \$ B \$ 3^{*} \$ B \$ 4^{*} \$ B \$ 5^{\star} \$ B \$ 6^{*} D 19^{*}$ Relocation!H19*O19 & $=\$ B \$ 7^{*} \$ D 19^{*}$ Relocation!E19*O19 & $=\$ B \$ 7^{*} \$ D 19^{*}$ Relocation $\$$ F $19^{*} \mathrm{O} 19$ \\
\hline 20 & $\mathrm{H}-3$ & $=$ Relocation!Y20 & $=\mathrm{C} 20 / \$ \mathrm{C} \$ 39$ & $=\$ B \$ 7^{*} \$ B \$ 3^{*} \$ B \$ 4^{*} \$ B \$ 5^{*} \$ B \$ 6^{*} D 20^{*}$ Relocation!H20*O20 & $=\$ B \$ 7^{*} \$ D 20^{*}$ Relocation!E20*O20 & $=\$ \mathrm{~B} \$ 7^{*} \$ D 20^{*}$ Relocation! $\$ F 20^{*} \mathrm{O} 20$ \\
\hline 21 & Np-237 & =Relocation!Y21 & $=\mathrm{C} 21 / \$ \mathrm{C} \$ 39$ & $=\$ B \$ 7^{*} \$ B \$ 3^{*} \$ B \$ 4^{*} \$ B \$ 5^{*} \$ B \$ 6^{*} D 21^{*}$ Relocation!H21*O21 & $=\$ B \$ 7^{*} \$ \mathrm{D} 21^{*}$ RelocationlE $21^{*} \mathrm{O} 21$ & $=\$ B \$ 7^{*} \$ \mathrm{D} 21^{\star}$ Relocation! $\$ \mathrm{~F} 21^{*} \mathrm{O} 21$ \\
\hline 22 & $\mathrm{Pm}-147$ & $=$ RelocationlY22 & $=\mathrm{C} 22 / \$ \mathrm{C} \$ 39$ & $=\$ B \$ 7^{\star} \$ B \$ 3^{*} \$ B \$ 4^{*} \$ B \$ 5^{*} \$ B \$ 6^{*} \mathrm{D} 22^{*}$ Relocation!H22* $\mathrm{O} 22$ & $=\$ B \$ 7^{*} \$ D 22^{*}$ Relocation!E22*O 022 & $=\$ B \$ 7^{*} \$ D 22^{*}$ Relocation! $\$ F 22^{*} \mathrm{O} 22$ \\
\hline 23 & Pu-238 & $=$ Relocation!Y23 & $=\mathrm{C} 23 / \$ \mathrm{C} \$ 39$ & $=\$ B \$ 7^{*} \$ B \$ 3^{*} \$ B \$ 4^{*} \$ B \$ 5^{*} \$ B \$ 6^{*} D 23^{*}$ Relocation!H23* 223 & $=\$ B \$ 7^{*} \$ D 23^{*}$ RelocationIE23*O23 & $=\$ B \$ 7^{*} \$ D 23^{*}$ Relocation $1 \$ F 23^{*} \mathrm{O} 23$ \\
\hline 24 & Pu-239 & $=$ Relocation!Y24 & $=\mathrm{C} 24 / \$ \mathrm{C} \$ 39$ & $=\$ B \$ 7^{*} \$ B \$ 3^{*} \$ B \$ 4^{*} \$ B \$ 5^{*} \$ B \$ 6^{*} D 24^{*}$ Relocation!H24* & $=\$ B \$ 7^{*} \$ D 24^{*}$ Relocation!E24*O24 & $=\$ B \$ 7^{*} \$ D 24^{*}$ Relocation! $\$ F 24^{*} \mathrm{O} 24$ \\
\hline 25 & Pu-239/241 & $=$ RelocationIY25 & $=\mathrm{C} 25 / \$ C \$ 39$ & & & \\
\hline 26 & Pu-240 & $=$ Relocation!Y26 & $=\mathrm{C} 26 / \$ \mathrm{C} \$ 39$ & $=\$ \mathrm{~B} \$ 7^{*} \$ \mathrm{~B} \$ 3^{*} \$ \mathrm{~B} \$ 4^{*} \$ \mathrm{~B} \$ 5^{*} \$ \mathrm{~B} \$ 6^{*} \mathrm{D} 26^{*}$ Relocation!H26*026 & $=\$ B \$ 7^{*} \$ \mathrm{D} 26^{*}$ Relocation!E26*026 & $=\$ B \$ 7^{*} \$ D 26^{*}$ Relocation! $\$ F 26^{*} \mathrm{O} 26$ \\
\hline 27 & Pu-241 & $=$ RelocationIY27 & $=\mathrm{C} 27 / \$ \mathrm{C} \$ 39$ & $=\$ B \$ 7^{*} \$ B \$ 3^{*} \$ B \$ 4^{*} \$ B \$ 5^{*} \$ B \$ 6^{*} \mathrm{D} 27^{*}$ Relocation!H27*027 & $=\$ B \$ 7^{*} \$ D 27^{\star}$ Relocation!E27*O27 & $=\$ B \$ 7^{*} \$ D 27^{*}$ Relocation! $\$ F 27^{*} \mathrm{O} 27$ \\
\hline 28 & Pu-242 & $=$ Relocation!Y28 & $=\mathrm{C} 28 / \$ \mathrm{C} \$ 39$ & $=\$ B \$ 7^{*} \$ B \$ 3^{*} \$ B \$ 4^{*} \$ B \$ 5^{*} \$ B \$ 6^{*} D 28^{*}$ Relocation $1 \mathrm{H} 28^{*} \mathrm{O} 28$ & $=\$ B \$ 7^{*} \$ D 28^{*}$ Relocation!E28*028 & $=\$ B \$ 7^{*} \$ D 28^{*}$ Relocation! $\$ F 28^{*} \mathrm{O} 28$ \\
\hline 29 & Sr-90 & =Relocation!Y29 & $=\mathrm{C} 29 / \$ \mathrm{C} \$ 39$ & $=\$ B \$ 7^{*} \$ B \$ 3^{*} \$ B \$ 4^{*} \$ B \$ 5^{*} \$ B \$ 6^{*} \mathrm{D} 29^{*}$ RelocationlH29*O29 & $=\$ B \$ 7^{*} \$ D 29^{*}$ Relocation!E29*O29 & $=\$ B \$ 7 * \$ D 29 *$ Relocation! $\$ F 29 * 029$ \\
\hline 30 & Tc-99 & $=$ Relocation!Y30 & $=\mathrm{C} 30 / \$ C \$ 39$ & $=\$ B \$ 7^{*} \$ B \$ 3^{*} \$ B \$ 4^{*} \$ B \$ 5^{*} \$ B \$ 6^{*} D 30^{*}$ Relocation!H30 & $=\$ B \$ 7^{*} \$ D 30^{*}$ Relocation!E30*O30 & $=\$ B \$ 7^{*} \$ D 30^{*}$ Relocation $\$$ F $30^{*} \mathrm{O} 30$ \\
\hline 31 & Th-232 & $=$ Relocation!Y31 & $=\mathrm{C} 31 / \$ C \$ 39$ & $=\$ B \$ 7^{*} \$ B \$ 3^{*} \$ B \$ 4^{*} \$ B \$ 5^{*} \$ B \$ 6^{*} D 31^{*}$ Relocation!H31*O 31 & $=\$ B \$ 7^{*} \$ D 31^{*}$ Relocation!E31*O31 & $=\$ B \$ 7 * \$ D 31^{*}$ Relocation! $\$ F 31^{*} \mathrm{O} 31$ \\
\hline 32 & TI-204 & $=$ Relocation!Y32 & $=\mathrm{C} 32 / \$ \mathrm{C} \$ 39$ & $=\$ B \$ 7^{*} \$ B \$ 3^{*} \$ B \$ 4^{*} \$ B \$ 5^{*} \$ B \$ 6^{*} D 32^{*}$ Relocation!H32*032 & $=\$ B \$ 7^{*} \$ D 32^{*}$ Relocation!E32*032 & $=\$ B \$ 7^{*} \$ D 32^{*}$ Relocation $\$$ F32*O32 \\
\hline 33 & U-235 & $=$ Relocation!Y33 & $=\mathrm{C} 33 / \$ \mathrm{C} \$ 39$ & $=\$ B \$ 7^{*} \$ B \$ 3^{*} \$ B \$ 4^{*} \$ B \$ 5^{*} \$ B \$ 6^{*} D 33^{*}$ Relocation!H33*O33 & $=\$ B \$ 7^{*} \$ D 33^{*}$ Relocation!E33*033 & $=\$ B \$ 7^{*} \$ D 33^{*}$ Relocation $\$ F 33^{*} \mathrm{O} 33$ \\
\hline 34 & U-238 & $=$ Relocation!Y34 & $=C 34 / \$ C \$ 39$ & $=\$ B \$ 7^{*} \$ B \$ 3^{*} \$ B \$ 4^{*} \$ B \$ 5^{*} \$ B \$ 6^{*} D 34^{*}$ Relocation!H34*O34 & $=\$ B \$ 7^{*} \$ D 34^{*}$ Relocation!E34*034 & $=\$ B \$ 7^{*} \$ D 34^{*}$ Relocation $1 \$ F 34^{*} \mathrm{O} 34$ \\
\hline 35 & & & & & & \\
\hline 36 & Ba-137m & $=$ RelocationIY36 & $=\mathrm{C} 36 / \$ C \$ 39$ & & & \\
\hline$\frac{37}{38}$ & $Y-90$ & $=$ RelocationIY37 & $=\mathrm{C} 37 / \$ \mathrm{C} \$ 39$ & & & \\
\hline$\frac{38}{39}$ & Total & $=$ SUM(C10:C38) & $\mathrm{M}(\mathrm{D} 10$ & & $=S U M(G 10: G 38)$ & $=\operatorname{SUM}(110: 138)$ \\
\hline & & & & & & \\
\hline
\end{tabular}




\begin{tabular}{|c|c|c|c|c|}
\hline & $\mathrm{J}$ & $\mathrm{K}$ & L & 으 \\
\hline 9 & BS Dose (mrem per uCl/m^2) & $\begin{array}{l}\text { Halflife } \\
\text { (years) }\end{array}$ & Lambda1 & Weathering Factor ( 50 years) \\
\hline 10 & $=\$ B \$ 7^{*} \$ B \$ 3^{*} \$ B \$ 4^{*} \$ B \$ 5^{*} \$ B \$ 6^{*} \$ D 10^{*}$ Relocation $\mid \$ 10^{*} 010$ & $=T$ _halific2 & $=0.693 / \mathrm{K} 10$ & $=\left(0.63 /(\$ L 10+\$ K \$ 5)^{*}\left(1-\operatorname{EXP}\left(-(\$ L 10+\$ K \$ 5)^{*} \$ 0 \$ 6\right)\right)\right)+\left((0.37 /(\$ L 10+\$ K \$ 7))^{*}\left(1-\operatorname{EXP}\left(-(\$ L 10+\$ K \$ 7)^{*} \$ 0 \$ 6\right)\right)\right)$ \\
\hline 11. & $=\$ B \$ 7^{*} \$ B \$ 3^{*} \$ B \$ 4^{*} \$ B \$ 5^{*} \$ B \$ 6^{*} \$ D 11^{*}$ Relocation! $\$ 111^{*} 011$ & $=T_{-}$half!C3 & $=0.693 / \mathrm{K} 11$ & $=\left(0.63 /(\$ L 11+\$ K \$ 5)^{*}\left(1-E X P\left(-(\$ L 11+\$ K \$ 5)^{*} \$ O \$ 6\right)\right)\right)+\left((0.37 /(\$ L 11+\$ K \$ 7))^{*}\left(1-\operatorname{EXP}\left(\cdot(\$ L 11+\$ K \$ 7)^{*} \$ O \$ 6\right)\right)\right)$ \\
\hline 12 & $=\$ B \$ 7^{*} \$ B \$ 3^{*} \$ B \$ 4^{*} \$ B \$ 5^{*} \$ B \$ 6 * \$ D 12^{*}$ Relocation! $\$ J 12^{*} 012$ & $=T$ half!C4 & $=0.693 / \mathrm{K} 12$ & $=\left(0.63 /(\$ L 12+\$ K \$ 5)^{*}\left(1-\operatorname{EXP}\left(-(\$ L 12+\$ K \$ 5)^{*} \$ O \$ 6\right)\right)\right)+\left((0.37 /(\$ L 12+\$ K \$ 7))^{*}\left(1-\operatorname{EXP}\left(-(\$ L 12+\$ K \$ 7)^{*} \$ O \$ 6\right)\right)\right)$ \\
\hline 13 & $=\$ B \$ 7^{\star} \$ B \$ 3^{*} \$ B \$ 4^{*} \$ B \$ 5^{*} \$ B \$ 6^{*} \$ D 13^{*}$ Relocation $1 \$ 13^{*} 013$ & $=T_{-}$half!C5 & $=0.693 / \mathrm{K} 13$ & $=\left(0.63 /(\$ L 13+\$ K \$ 5)^{*}\left(1-\operatorname{EXP}\left(-(\$ L 13+\$ K \$ 5)^{*} \$ O \$ 6\right)\right)\right)+\left((0.37 /(\$ L 13+\$ K \$ 7))^{*}\left(1-\operatorname{EXP}\left(-(\$ L 13+\$ K \$ 7)^{*} \$ O \$ 6\right)\right)\right)$ \\
\hline 14. & $=\$ B \$ 7^{*} \$ B \$ 3^{*} \$ B \$ 4^{*} \$ B \$ 5^{*} \$ B \$ 6^{*} \$ D 14^{*}$ Relocation $1 \$ 114^{*}$ O 14 & $=T_{-}$half!C6 & $=0.693 / \mathrm{K} 14$ & $=\left(0.63 /(\$ L 14+\$ K \$ 5)^{*}\left(1-\operatorname{EXP}\left(-(\$ L 14+\$ K \$ 5)^{*} \$ 0 \$ 6\right)\right)\right)+\left((0.37 /(\$ L 14+\$ K \$ 7))^{*}\left(1-\operatorname{EXP}\left(-(\$ L 14+\$ K \$ 7)^{*} \$ O \$ 6\right)\right)\right)$ \\
\hline 15. & $=\$ B \$ 7^{*} \$ B \$ 3^{*} \$ B \$ 4^{*} \$ B \$ 5^{*} \$ B \$ 6^{*} \$ D 15^{*}$ Relocation $\$ \$ 15^{*} 015$ & $=T_{\text {_h half } ! C 7}$ & $=0.693 / \mathrm{K} 15$ & $\left.=\left(0.63 /(\$ L 15+\$ K \$ 5)^{*}\left(1-\operatorname{EXP}\left(-(\$ L 15+\$ K \$ 5)^{*} \$ O \$ 6\right)\right)\right)+\left((0.37 /(\$ L 15+\$ K \$ 7))^{*}\left(1-\operatorname{EXP}\left(\cdot(\$ L 15+\$ K \$ 7)^{*} \$ O \$ 6\right)\right)\right)\right)$ \\
\hline 16 & $=\$ B \$ 7^{*} \$ B \$ 3^{*} \$ B \$ 4^{*} \$ B \$ 5^{*} \$ B \$ 6^{*} \$ D 16^{*}$ Pelocation $\$ \$ 16^{*} 016$ & $=T_{-}$halfic8 & $=0.693 / \mathrm{K} 16$ & $=\left(0.63 /(\$ L 16+\$ K \$ 5)^{*}\left(1-\operatorname{EXP}\left(-(\$ L 16+\$ K \$ 5)^{*} \$ O \$ 6\right)\right)\right)+\left((0.37 /(\$ L 16+\$ K \$ 7))^{*}\left(1-\operatorname{EXP}\left(-(\$ L 16+\$ K \$ 7)^{*} \$ O \$ 6\right)\right)\right)$ \\
\hline 17. & $=\$ B \$ 7^{*} \$ B \$ 3^{*} \$ B \$ 4^{*} \$ B \$ 5^{*} \$ B \$ 6^{*} \$ D 17^{*}$ Relocation $! \$ J 17^{*} 017$ & $=T \_$halfiC9 & $=0.693 / \mathrm{K} 17$ & $=\left(0.63 /(\$ L 17+\$ K \$ 5)^{*}\left(1-\operatorname{EXP}\left(-(\$ L 17+\$ K \$ 5)^{*} \$ O \$ 6\right)\right)\right)+\left((0.37 /(\$ L 17+\$ K \$ 7))^{*}\left(1-\operatorname{EXP}\left(-(\$ L 17+\$ K \$ 7)^{*} \$ O \$ 6\right)\right)\right)$ \\
\hline 18 & $=\$ B \$ 7^{*} \$ B \$ 3^{*} \$ B \$ 4^{*} \$ B \$ 5^{*} \$ B \$ 6^{*} \$ D 18^{*}$ Relocation $\$ J 18^{*} 018$ & $=T_{-}$halfIC10 & $=0.693 / \mathrm{K} 18$ & $=\left(0.63 /(\$ L 18+\$ K \$ 5)^{*}\left(1-\operatorname{EXP}\left(-(\$ L 18+\$ K \$ 5)^{*} \$ O \$ 6\right)\right)\right)+\left((0.37 /(\$ L 18+\$ K \$ 7))^{*}\left(1-\operatorname{EXP}\left(-(\$ L 18+\$ K \$ 7)^{*} \$ O \$ 6\right)\right)\right)$ \\
\hline 19 & $=\$ B \$ 7^{*} \$ B \$ 3^{*} \$ B \$ 4^{*} \$ B \$ 5^{*} \$ B \$ 6^{*} \$ D 19^{*}$ Relocation $\$ J 19^{*} 019$ & $=T_{-}$halfIC11 & $=0.693 / \mathrm{K} 19$ & $=\left(0.63 /(\$ L 19+\$ K \$ 5)^{\star}\left(1-\operatorname{EXP}\left(-(\$ L 19+\$ K \$ 5)^{*} \$ 0 \$ 6\right)\right)\right)+\left((0.37 /(\$ L 19+\$ K \$ 7))^{*}\left(1-\operatorname{EXP}\left(-(\$ L 19+\$ K \$ 7)^{*} \$ O \$ 6\right)\right)\right)$ \\
\hline 20 & $=\$ B \$ 7^{*} \$ B \$ 3^{*} \$ B \$ 4^{*} \$ B \$ 5^{*} \$ B \$ 6^{*} \$ D 20^{*}$ Relocation $1 \$ 20^{*} 020$ & $=T$ _half!C12 & $=0.693 / \mathrm{K} 20$ & $=\left(0.63 /(\$ L 20+\$ K \$ 5)^{*}\left(1-\operatorname{EXP}\left(-(\$ L 20+\$ K \$ 5)^{*} \$ O \$ 6\right)\right)\right)+\left((0.37 /(\$ L 20+\$ K \$ 7))^{*}\left(1-\operatorname{EXP}\left(-(\$ L 20+\$ K \$ 7)^{*} \$ O \$ 6\right)\right)\right)$ \\
\hline 21 & $=\$ B \$ 7^{*} \$ B \$ 3^{*} \$ B \$ 4^{*} \$ B \$ 5^{*} \$ B \$ 6^{*} \$ D 21^{*}$ Relocation $1 \$ 21^{*} \mathrm{O} 21$ & $=T \_$half!C13 & $=0.693 / \mathrm{K} 21$ & $=\left(0.63 /(\$ L 21+\$ K \$ 5)^{*}\left(1-\operatorname{EXP}\left(-(\$ L 21+\$ K \$ 5)^{*} \$ 0 \$ 6\right)\right)\right)+\left((0.37 /(\$ L 21+\$ K \$ 7))^{*}\left(1-\operatorname{EXP}\left(-(\$ L 21+\$ K \$ 7)^{*} \$ O \$ 6\right)\right)\right)$ \\
\hline 22 & $=\$ B \$ 7^{*} \$ B \$ 3^{*} \$ B \$ 4^{*} \$ B \$ 5^{*} \$ B \$ 6^{*} \$ D 22^{*}$ Relocation! $\$ J 22^{*} \mathrm{O} 22$ & $=T$ halfic14 & $=0.693 / \mathrm{K} 22$ & $=\left\{0.63 /(\$ L 22+\$ K \$ 5)^{*}\left(1-\operatorname{EXP}\left(-(\$ L 22+\$ K \$ 5)^{*} \$ 0 \$ 6\right)\right)\right)+\left((0.37 /(\$ L 22+\$ K \$ 7))^{*}\left(1-\operatorname{EXP}\left(-(\$ L 22+\$ K \$ 7)^{*} \$ 0 \$ 6\right)\right)\right)$ \\
\hline 23 & $=\$ B \$ 7^{*} \$ B \$ 3^{*} \$ B \$ 4^{*} \$ B \$ 5^{*} \$ B \$ 6^{*} \$ D 23^{*}$ Relocation $1 \$ J 23^{*} \mathrm{O} 23$ & $=T$ _halfIC15 & $=0.693 / \mathrm{K} 23$ & $=\left(0.63 /(\$ L 23+\$ K \$ 5)^{*}\left(1-\operatorname{EXP}\left(-(\$ L 23+\$ K \$ 5)^{*} \$ 0 \$ 6\right)\right)\right)+\left((0.37 /(\$ L 23+\$ K \$ 7))^{*}\left(1-\operatorname{EXP}\left(-(\$ L 23+\$ K \$ 7)^{*} \$ O \$ 6\right)\right)\right)$ \\
\hline$\frac{24}{25}$ & $=\$ B \$ 7^{*} \$ B \$ 3^{*} \$ B \$ 4^{*} \$ B \$ 5^{*} \$ B \$ 6^{*} \$ D 24^{*}$ Relocation $1 \mathrm{~J} 24^{*} \mathrm{O} 24$ & $=T_{\text {_h halfIC16 }}$ & $=0.693 / \mathrm{K} 24$ & $=\left(0.63 /(\$ L 24+\$ K \$ 5)^{*}\left(1-\operatorname{EXP}\left(-(\$ L 24+\$ K \$ 5)^{*} \$ 0 \$ 6\right)\right)\right)+\left((0.37 /(\$ L 24+\$ K \$ 7))^{*}\left(1-\operatorname{EXP}\left(-(\$ L 24+\$ K \$ 7)^{*} \$ 0 \$ 6\right)\right)\right)$ \\
\hline 26 & $=\$ B \$ 7^{*} \$ B \$ 3^{*} \$ B \$ 4^{*} \$ B \$ 5^{*} \$ B \$ 6^{*} \$ D 26^{*}$ Relocation! $\$ J 26^{*} \mathrm{O} 26$ & $=T_{\text {_half } I C 18}$ & $=0.693 / \mathrm{K} 26$ & $=\left(0.63 /(\$ L .26+\$ K \$ 5)^{*}\left(1-\operatorname{EXP}\left(-(\$ L 26+\$ K \$ 5)^{*} \$ 0 \$ 6\right)\right)\right)+\left((0.37 /(\$ L 26+\$ K \$ 7))^{*}\left(1-\operatorname{EXP}\left(-(\$ L 26+\$ K \$ 7)^{*} \$ O \$ 6\right)\right)\right)$ \\
\hline 27 & $=\$ B \$ 7^{*} \$ B \$ 3^{*} \$ B \$ 4^{*} \$ B \$ 5^{*} \$ B \$ 6^{*} \$ D 27^{*}$ Relocation!\$J27*027 & $=T$ _halfic19 & $=0.693 / \mathrm{K} 27$ & $=\left(0.63 /(\$ L 27+\$ K \$ 5)^{*}\left(1-\operatorname{EXP}\left(-(\$ L 27+\$ K \$ 5)^{*} \$ 0 \$ 6\right)\right)\right)+\left((0.37 /(\$ L 27+\$ K \$ 7))^{*}\left(1-\operatorname{EXP}\left(-(\$ L 27+\$ K \$ 7)^{*} \$ O \$ 6\right)\right)\right)$ \\
\hline 28 & $=\$ B \$ 7 * B 3^{*} \$ B \$ 4^{*} \$ B \$ 5^{*} \$ B \$ 6^{*} \$ D 28^{*}$ Relocation $1 \$ 28^{*} \mathrm{O} 28$ & $=T$ half $! C 20$ & $=0.693 / \mathrm{K} 28$ & $=\left(0.63 /(\$ L 28+\$ K \$ 5)^{*}\left(1-\operatorname{EXP}\left(-(\$ L 28+\$ K \$ 5)^{*} \$ O \$ 6\right)\right)\right)+\left((0.37 /(\$ L 28+\$ K \$ 7))^{*}\left(1-\operatorname{EXP}\left(-(\$ L 28+\$ K \$ 7)^{*} \$ O \$ 6\right)\right)\right)$ \\
\hline 29 & $=\$ B \$ 7^{*} \$ B \$ 3^{*} \$ B \$ 4^{*} \$ B \$ 5^{*} \$ B \$ 6^{*} \$ D 29^{*}$ Relocation $\mid \$ J 29^{*} \mathrm{O} 29$ & $=T_{\text {_ half }} \mathrm{C} 21$ & $=0.693 / \mathrm{K} 29$ & $=\left(0.63 /(\$ L 29+\$ K \$ 5)^{*}\left(1-\operatorname{EXP}\left(-(\$ L 29+\$ K \$ 5)^{*} \$ O \$ 6\right)\right)\right)+\left((0.37 /(\$ L 29+\$ K \$ 7))^{*}\left(1-\operatorname{EXP}\left(-(\$ L 29+\$ K \$ 7)^{*} \$ O \$ 6\right)\right)\right)$ \\
\hline 30 & $=\$ B \$ 7^{*} \$ B \$ 3^{*} \$ B \$ 4^{*} \$ B \$ 5^{*} \$ B \$ 6^{*} \$ D 30^{*}$ Relocation! $\$ J 30^{*} 030$ & $=T$ half!C22 & $=0.693 / K 30$ & $=\left(0.63 /(\$ L 30+\$ K \$ 5)^{*}\left(1-\operatorname{EXP}\left(-(\$ L 30+\$ K \$ 5)^{*} \$ 0 \$ 6\right)\right)\right)+\left((0.37 /(\$ L 30+\$ K \$ 7))^{*}\left(1-\operatorname{EXP}\left(-(\$ L 30+\$ K \$ 7)^{*} \$ O \$ 6\right)\right)\right)$ \\
\hline 31 & $=\$ B \$ 7^{*} \$ B \$ 3^{*} \$ B \$ 4^{*} \$ B \$ 5^{*} \$ B \$ 6^{*} \$ D 31^{*}$ Relocation $\mid \$ J 31^{*} 031$ & $=T$ _half!C23 & $=0.693 / \mathrm{K} 31$ & $=\left(0.63 /(\$ L 31+\$ K \$ 5)^{*}\left(1-\operatorname{EXP}\left(-(\$ L 31+\$ K \$ 5)^{*} \$ 0 \$ 6\right)\right)\right)+\left((0.37 /(\$ L 31+\$ K \$ 7))^{*}\left(1-\operatorname{EXP}\left(-(\$ L 31+\$ K \$ 7)^{*} \$ O \$ 6\right)\right)\right)$ \\
\hline 32 & $=\$ B \$ 7^{*} \$ B \$ 3^{*} \$ B \$ 4^{*} \$ B \$ 5^{*} \$ B \$ 6^{*} \$ D 32^{*}$ Relocation $\$$ \$ J32*032 & $=T \_$halflC24 & $=0.693 / \mathrm{K} 32$ & $=\left(0.63 /(\$ L 32+\$ K \$ 5)^{*}\left(1-\operatorname{EXP}\left(\cdot(\$ L 32+\$ K \$ 5)^{*} \$ O \$ 6\right)\right)\right)+\left((0.37 /(\$ L 32+\$ K \$ 7))^{*}\left(1-\operatorname{EXP}\left(-(\$ L 32+\$ K \$ 7)^{*} \$ O \$ 6\right)\right)\right)$ \\
\hline 33 & $=\$ B \$ 7^{*} \$ B \$ 3^{*} \$ B \$ 4^{*} \$ B \$ 5^{*} \$ B \$ 6^{*} \$ D 33^{*}$ Relocationl\$J33*O33 & $=T \_$half!C25 & $=0.693 / \mathrm{K} 33$ & $=\left(0.63 /(\$ L 33+\$ K \$ 5)^{*}\left(1-\operatorname{EXP}\left(-(\$ L 33+\$ K \$ 5)^{*} \$ 0 \$ 6\right)\right)\right)+\left((0.37 /(\$ L 33+\$ K \$ 7))^{*}\left(1-\operatorname{EXP}\left(-(\$ L 33+\$ K \$ 7)^{*} \$ 0 \$ 6\right)\right)\right)$ \\
\hline \begin{tabular}{|l|}
34 \\
5
\end{tabular} & $=\$ B \$ 7^{*} \$ B \$ 3^{*} \$ B \$ 4^{*} \$ B \$ 5^{*} \$ B \$ 6^{*} \$ D 34^{*}$ Relocation! $\$ J 34^{*} 034$ & $=T_{\text {_half }} ! \mathrm{C} 26$ & $=0.693 / K 34$ & $=\left(0.63 /(\$ L 34+\$ K \$ 5)^{*}\left(1-\operatorname{EXP}\left(-(\$ L 34+\$ K \$ 5)^{*} \$ O \$ 6\right)\right)\right)+\left((0.37 /(\$ L 34+\$ K \$ 7))^{*}\left(1-\operatorname{EXP}\left(-(\$ L 34+\$ K \$ 7)^{*} \$ O \$ 6\right)\right)\right)$ \\
\hline 36 & & $=T \_$half $! \mathrm{C} 28$ & $=0.693 / \mathrm{K} 36$ & $=\left(0.63 /(\$ L 36+\$ K \$ 5)^{*}\left(1-\operatorname{EXP}\left(-(\$ L 36+\$ K \$ 5)^{*} \$ 0 \$ 6\right)\right)\right)+\left((0.37 /(\$ L 36+\$ K \$ 7))^{*}\left(1-\operatorname{EXP}\left(-(\$ L 36+\$ K \$ 7)^{*} \$ O \$ 6\right)\right)\right)$ \\
\hline 37 & & $=T \_$half!C29 & $=0.693 / \mathrm{K} 37$ & $=\left(0.63 /(\$ L 37+\$ K \$ 5)^{*}\left(1-\operatorname{EXP}\left(-(\$ L 37+\$ K \$ 5)^{*} \$ 0 \$ 6\right)\right)\right)+\left((0.37 /(\$ L 37+\$ K \$ 7))^{*}\left(1-\operatorname{EXP}\left(-(\$ L 37+\$ K \$ 7)^{*} \$ O \$ 6\right)\right)\right)$ \\
\hline 38 & & & & \\
\hline$\frac{39}{40}$ & $=S U M(J 10: \sqrt{38})$ & & & \\
\hline
\end{tabular}




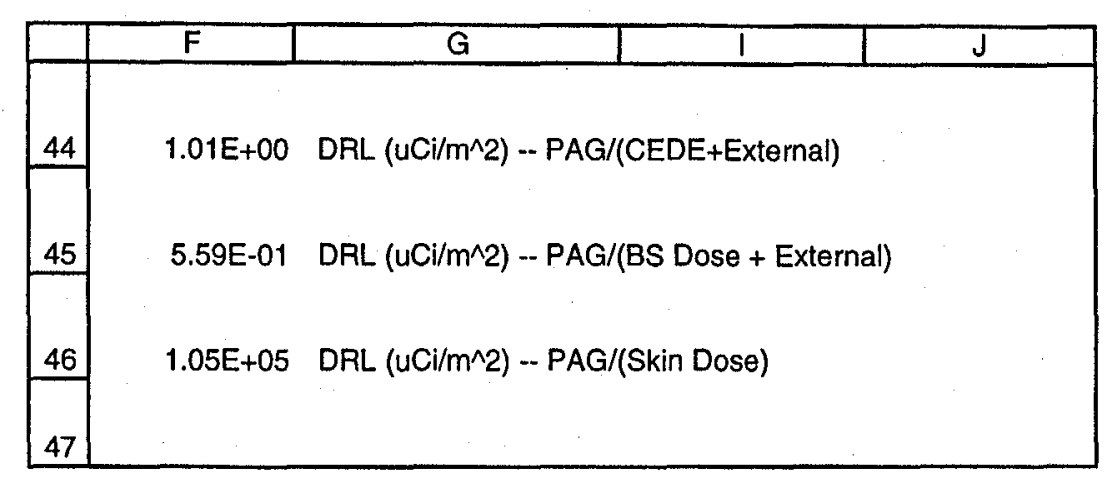




\begin{tabular}{|c|c|c|c|}
\hline & $\mathbf{F}$ & $\bar{G}$ & $T$ \\
\hline 44 & $=F 3 /(F 39+G 39)$ & DRL (uCi/m^2) -- PAG/(CEDE+External) & \\
\hline 45 & $=F 4 /(J 39+G 39)$ & DRL (uCi/m^2) - PAG/(BS Dose + External) & \\
\hline 46 & $=F 5 / 139$ & DRL $\left(u \mathrm{Ci}^{\prime} / \mathrm{m}^{\wedge} 2\right)$-- PAG/(Skin Dose) & \\
\hline 47 & & & \\
\hline
\end{tabular}




\begin{tabular}{|c|c|c|}
\hline & $B$ & C \\
\hline 1 & Nuclide & Halflife (years) \\
\hline 2 & Am-241 & 458 \\
\hline 3 & Am-243 & 7950 \\
\hline 4 & $\mathrm{Ba}-133$ & 10.5 \\
\hline 5 & $\mathrm{Ce}-144$ & 0.778082192 \\
\hline 6 & Cf-249 & 360 \\
\hline 7 & Cf-252 & 2.646 \\
\hline 8 & $\mathrm{Cm}-244$ & 17.6 \\
\hline 9 & $\mathrm{Cm}-246$ & 5500 \\
\hline 10 & Co-60 & 5.26 \\
\hline 11 & Cs-137 & 30 \\
\hline 12 & $\mathrm{H}-3$ & 12.3 \\
\hline 13 & $\mathrm{~Np}-237$ & 2140000 \\
\hline 14 & Pm-147 & 2.62 \\
\hline 15 & Pu-238 & 86.4 \\
\hline 16 & Pu-239 & 24390 \\
\hline 17 & Pu mix-239/241 & \\
\hline 18 & Pu-240 & 6580 \\
\hline 19 & Pu-241 & 13.2 \\
\hline 20 & Pu pure-242 & 379000 \\
\hline 21 & Sr-90 & 28.1 \\
\hline 22 & Tc-99 & 212000 \\
\hline 23 & Th-232 & 14000000000 \\
\hline 24 & TI-204 & $\begin{array}{ll}\text { : } \quad 3.8 \\
\end{array}$ \\
\hline 25 & |U-235 & 710000000 \\
\hline 26 & U.238 & 4510000000 \\
\hline 27 & & \\
\hline 28 & Ba-137m & $4.8554 \mathrm{E}-06$ \\
\hline 29 & |Y-90 & 0.007317352 \\
\hline 30 & Pr-144 & 3.28767E-05 \\
\hline 31 & Pa-233 & 0.073972603 \\
\hline 32 & Th-234 & 0.066027397 \\
\hline 33 & $\mathrm{~Pa}-234$ & 0.00076484 \\
\hline 34 & Np-239 & 0.006452055 \\
\hline 35 & Th-231 & 0.002913242 \\
\hline 36 & Ra-228 & 5.75 \\
\hline 37 & AC-228 & 0.000699772 \\
\hline 38 & & \\
\hline
\end{tabular}


$=$ IF(Relocation!\$D 10="y", Relocation!\$C10,IF(Relocation!\$D10="d",Relocation!\$C10/365,IF(Relocation!\$D10="h",Relocation!\$C10/(365"24),IF(Relocation!\$D10="m",Relocation!\$C10/(365*24*60),"error"))) $=I F($ Relocation!\$D11="y", Relocation!\$C11,IF(Relocation!\$D11 ="d",Relocation!\$C11/365,IF(Relocation!\$D11="h",Relocationl\$C11/(365*24),IF(Relocation!\$D11="m",Relocation!\$C11/(365*24*60),"error")))) =IF(Relocation!\$D12="y", Relocation!\$C12,IF(Relocation!\$D12="d",Relocationl\$C12/365,IF(Relocation!\$D12="h",Relocation!\$C12/(365*24),IF(Relocation!\$D12="m",Relocation!\$C12/(365*24*60),"error")))) =IF(Relocation!\$D13="y", Relocation!\$C13,IF(Relocation!\$D13="d",Relocation!\$C13/365,IF(Relocation!\$D13="h",Relocation!\$C13/(365*24),IF(Relocation!\$D13="m",Relocation!\$C13/(365"24*60),"error"))) =IF(Relocation!\$D14="y", Relocation!\$C14,IF(Relocation!\$D14="d",Relocation!\$C14/365,IF(Relocation!\$D14="h", Relocation!\$C14/(365*24),IF(Relocation!\$D14="m",Relocation!\$C14/(365*24*60),"error")))) $=$ IF(Relocation!\$D15="y", Relocation!\$C15,IF(Relocation!\$D15="d",Relocation!\$C15/365,IF(Relocationl\$D15="h",Relocation!\$C15/(365*24),IF(Relocation!\$D15="m",Relocation!\$C15/(365*24*60),"error")))) $=I F($ Relocation!\$D16="y", Relocation $\$ C 16, I F($ Relocation!\$D16="d",Relocationl\$C16/365,IF(Relocation!\$D16="h",Relocation!\$C16/(365*24),IF(Relocation!\$D16="m",Relocation!\$C16/(365*24*60),"error"))) $=$ IF (Relocation!\$D 17="y", Relocation!\$C17,IF(Relocation!\$D17="d", Relocation!\$C17/365,IF(Relocationl\$D 17="h",Relocationl\$C17/(365*24),IF(Relocation!\$D 17="mu",Relocation!\$C17/(365*24*60),"error")))

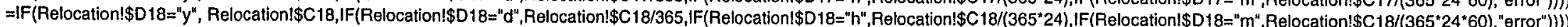
=IF(Relocation!\$D 19="y", Relocation!\$C19,IF(Relocation!\$D19="d",Relocationl\$C19/365,IF(Relocation!\$D 19="h", Relocationl\$C19/(365*24)IF(Relocationl\$D19="m" RelocationI\$C19/(365*24*60),"

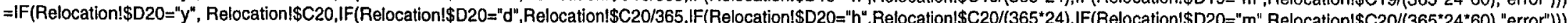

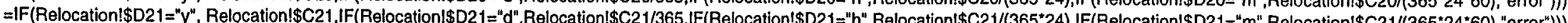

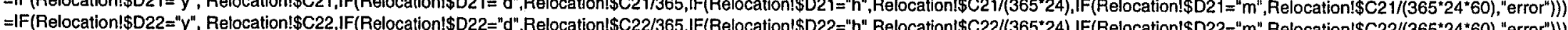

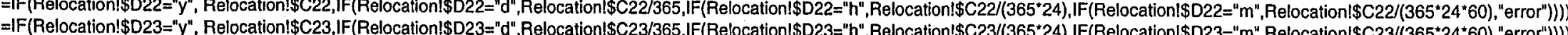
$=$ =IF(Relocation!\$D23="y", Relocation!\$C23,IF(Relocation!\$D23="d",Relocation!\$C23/365,IF(Relocation!\$D23="h",Relocation!\$C23/(365"24),IF(Relocation!\$D23="m",Relocation!\$C23/(365*24*60),"error"))) $=I F($ Relocation!\$D24="y", Relocation!\$C24,IF(Relocation!\$D24="d", Relocation!\$C24/365,IF(Relocation!\$D24="h",Relocationl\$C24/(365*24),IF(Relocation!\$D24="m",Relocation!\$C24/(365*24*60),"error"))) $=1 F\left(\right.$ Relocation $\$ D 26=" y^{n}$, Relocation!\$C26,IF(Relocation!\$D26="d",Relocation!\$C26/365,IF(Relocation!\$D26="h",Relocation!\$C26/(365*24),IF(Relocation!\$D26="m",Relocation! $\$ C 26 /\left(365 * 24^{*} 60\right), "$ error"))) =IF(Relocation!\$D27="y", Relocation!\$C27,IF(Relocation!\$D27="d", Relocation!\$C27/365,IF(Relocation!\$D27="h", Relocation!\$C27/(365"24),IF(Relocation!\$D27="m", Relocation!\$C27/(365*24"60),"error"))) =IF(Relocation!\$D28="y", Relocation!\$C28,IF(Relocation!\$D28="d",Relocation!\$C28/365,IF(Relocation!\$D28="h", Relocation!\$C28/(365*24),IF(Relocation!\$D28="m",Relocation!\$C28/(365*24*60),"error"))), =IF(Relocation!\$D29="y", Relocation!\$C29,IF(Relocation!\$D29="d",Relocation!\$C29/365,IF(Relocation!\$D29="h", Relocation!\$C29/(365*24),IF(Relocation!\$D29="m",Relocation!\$C29/(365*24*60) "error"))),

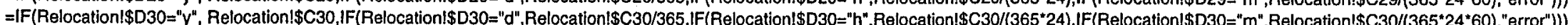

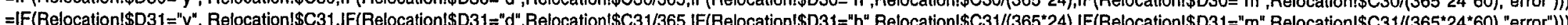
$=$ = F $($ Re $=$ =IF(Relocions

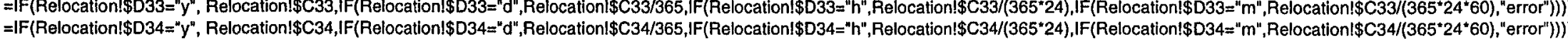

=IF(Relocation!\$D36="y", Relocationl\$C36,IF(Relocation!\$D36="d",Relocation!\$C36/365,IF(Relocation!\$D36=" $h "$, Relocation!\$C36/(365*24),IF(Relocation!\$D36="m",Relocation!\$C36/(365*24*60),"error"))) =IF(Relocation!\$D37="y", Relocation!\$C37,IF(Relocation!\$D37="d",Relocation!\$C37/365,IF(Relocation!\$D37="h",Relocation!\$C37/(365*24),IF(Relocation!\$D37="m",Relocation!\$C37/(365*24*60),"error"))) =IF(Relocation!\$D38="y", Relocation!\$C38,IF(Relocation!\$D38="d",Relocation!\$C38/365,IF(Relocation!\$D38="h",Relocation!\$C38/(365*24),IF(Relocation!\$D38="m",Relocation!\$C38/(365*24"60),"error"))), =IF(Relocation!\$D39="y", Relocation!\$C39,IF(Relocation!\$D39="d",Relocation!\$C39/365,IF(Relocation!\$D39=" h",Relocation!\$C39/(365*24),IF(Relocation!\$D39="m",Relocation!\$C39/(365*24*60), "error"))) $=$ IF (Relocation!\$D40="y", Relocation!\$C40,IF(Relocationl\$D40="d",Relocation!\$C40/365,IF(Relocation!\$D40="h", Relocation!\$C40/(365"24),IF(Relocation!\$D40="m",Relocation!\$C40/(365*24"60),"error"))) $=1 F(\mathrm{R})$

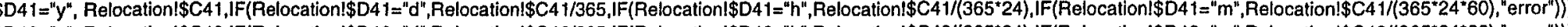
=IF(Relocation!\$D42="y", Relocation!\$C42,IF(Relocation!\$D42="d",Relocation!\$C42/365,IF(Relocation!\$D42="h",Relocation!\$C42/(365"24),IF(Relocation!\$D42="m",Relocation!\$C42/(365*24*60),"error"))) $=\mid F($ Reiocation!\$D43="y", Relocation!\$C43,IF(Relocation!\$D43="d",Relocation!\$C43/365,IF(Relocation!\$D43="h",Relocation!\$C43/(365*24),IF(Relocation!\$D43="m",Relocation!\$C43/(365*24*60),"error"))) $=$ IF (Relocation!\$D44="y", Relocation!\$C44,IF(Relocation $\$ D 44=" d "$, Relocation!\$C44/365,IF(Relocation!\$D44="h",Relocationl\$C44/(365*24),IF(Relocation!\$D44="m",Relocation!\$C44/(365*24*60),"error"))) $=$ IF (Relocation!\$D45="y", Relocation!\$C45,IF(Relocation!\$D45="d",Relocation!\$C45/365,IF(Relocation!\$D45="h",Relocation!\$C45/(365*24),IF(Relocation!\$D45="m",Relocation!\$C45/(365*24 "60),"error"))) 


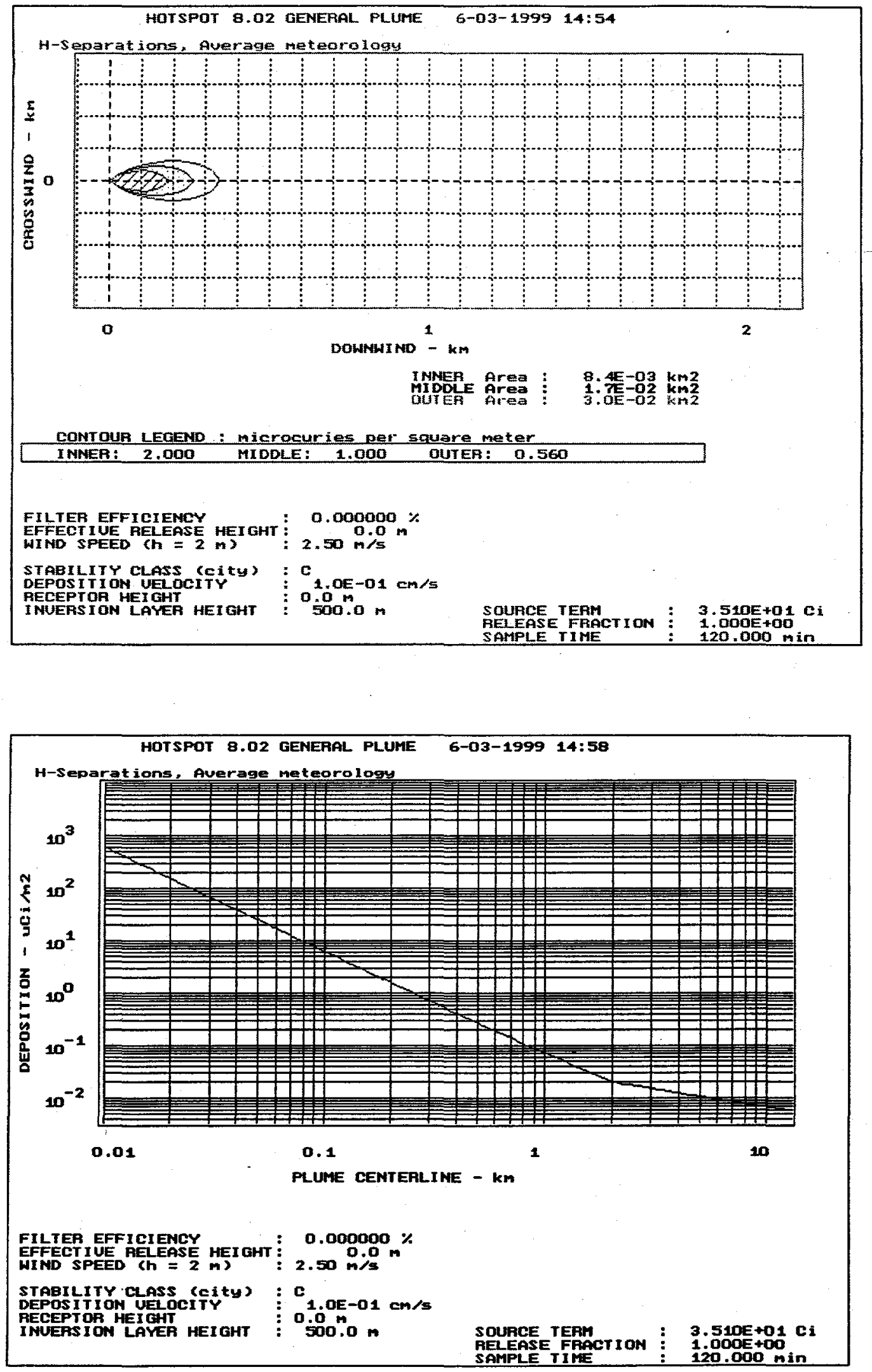


\section{Attachment C}

HOTSPOT results - average meteorology, $1.0 \mathrm{~cm} \mathrm{~s}^{-1}$ 

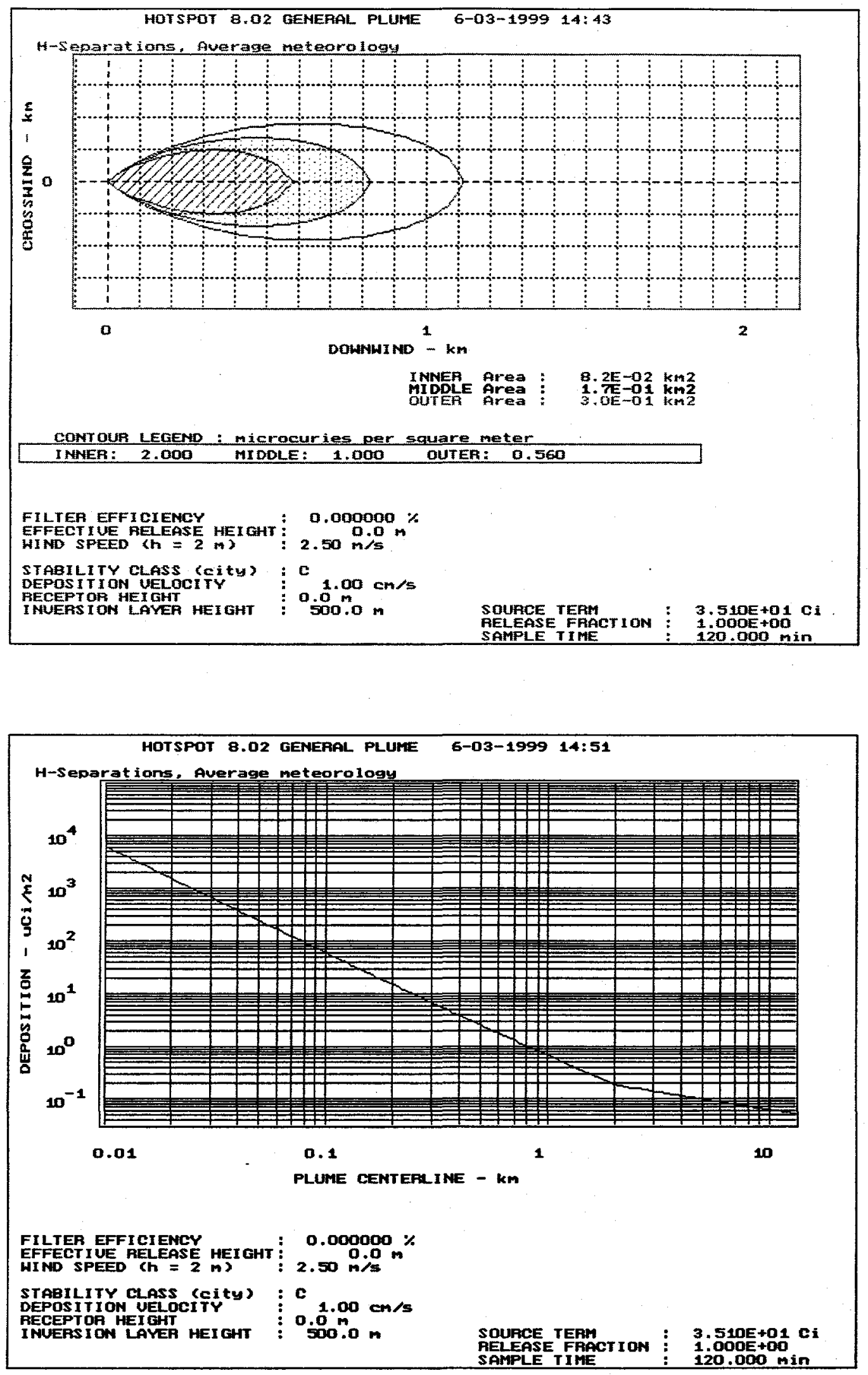


\section{Attachment D}

HOTSPOT results - average meteorology, $10 \mathrm{~cm} \mathrm{~s}^{-1}$ 

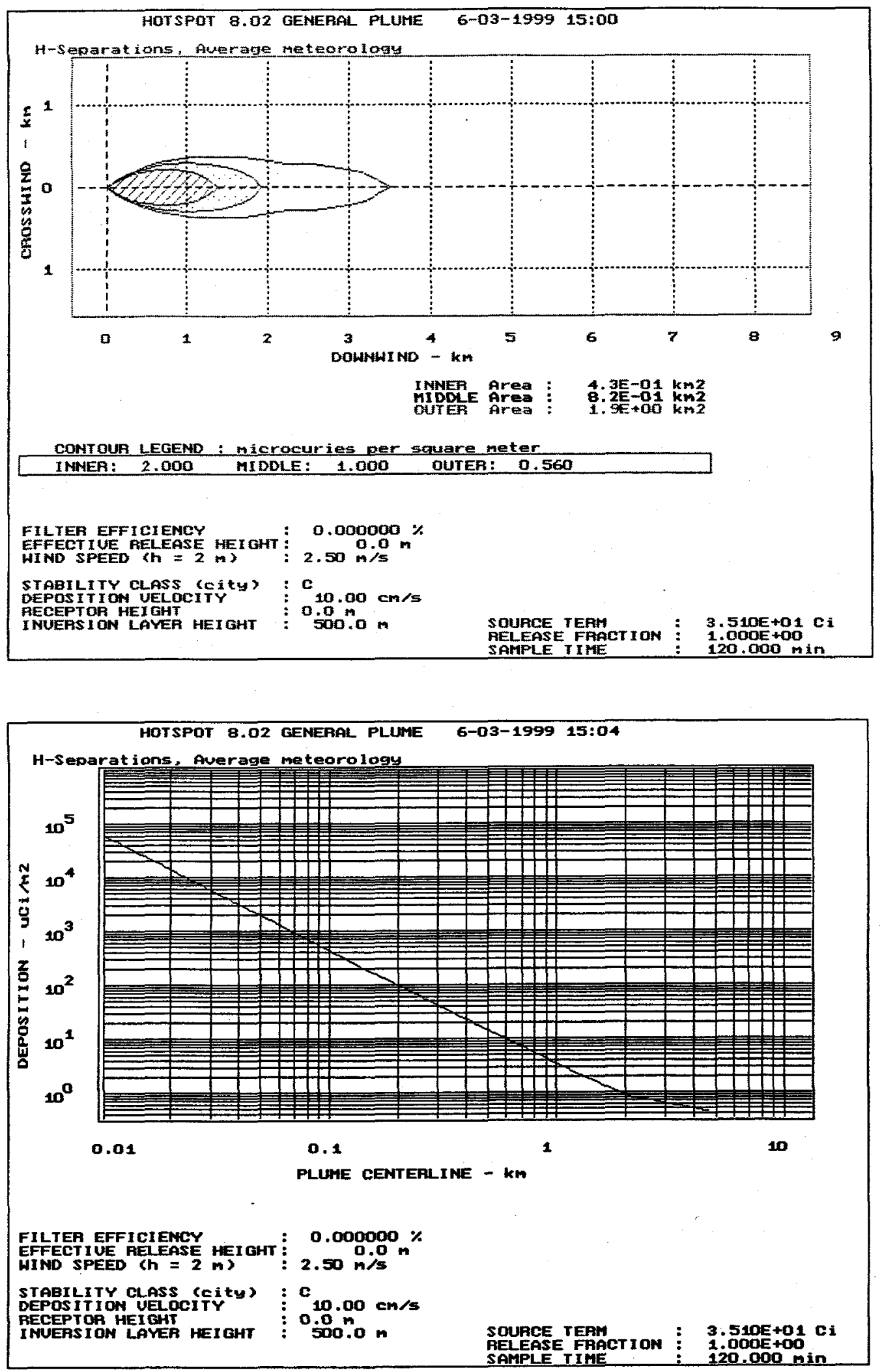
Relocation Impacts of an H-Separations Coil and Tube Failure (U)

\section{Attachment E}

HOTSPOT results - adverse meteorology, $0.1 \mathrm{~cm} \mathrm{~s}^{-1}$ 

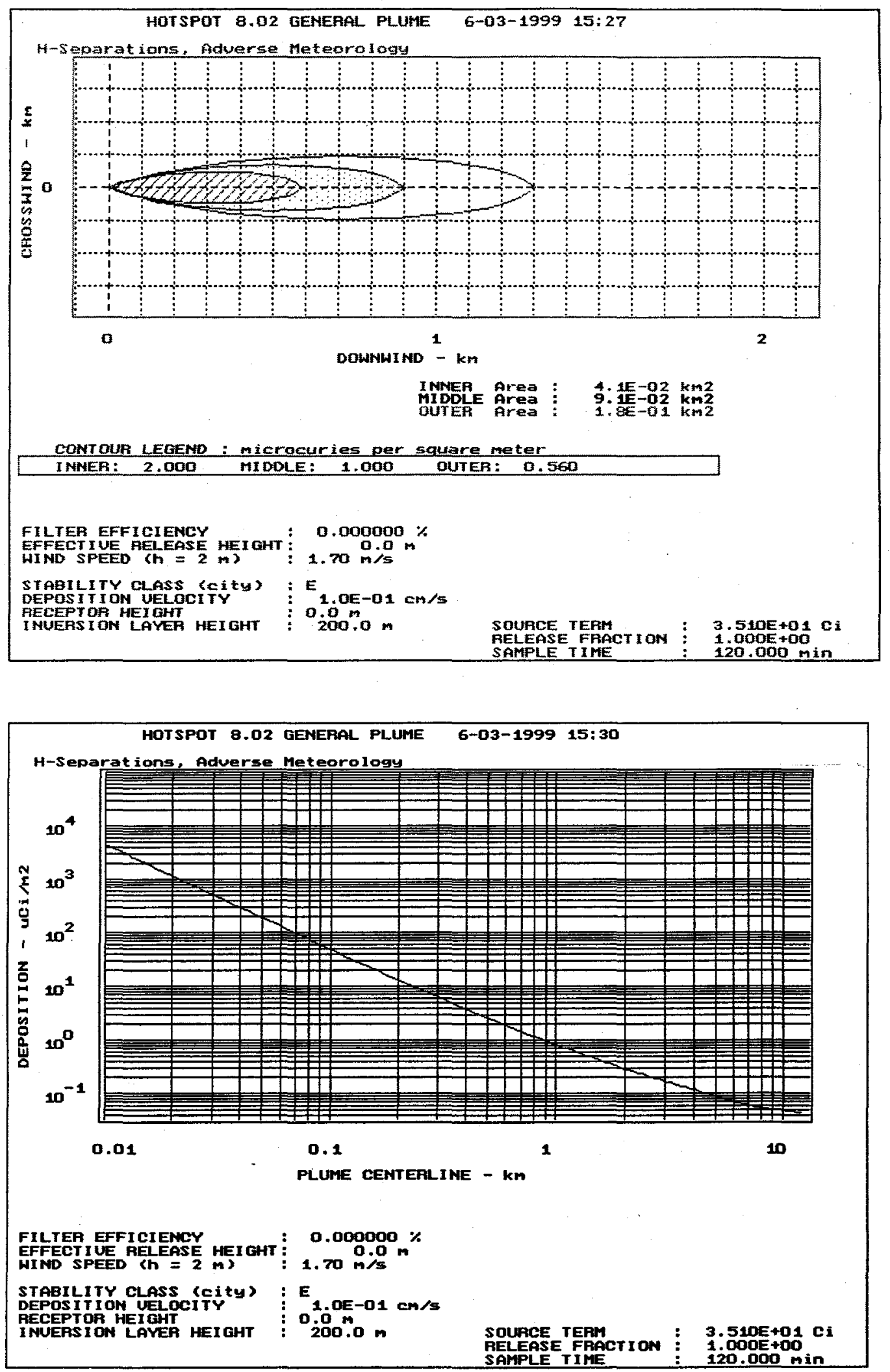


\section{Attachment F}

HOTSPOT results - adverse meteorology, $1.0 \mathrm{~cm} \mathrm{~s}^{-1}$ 

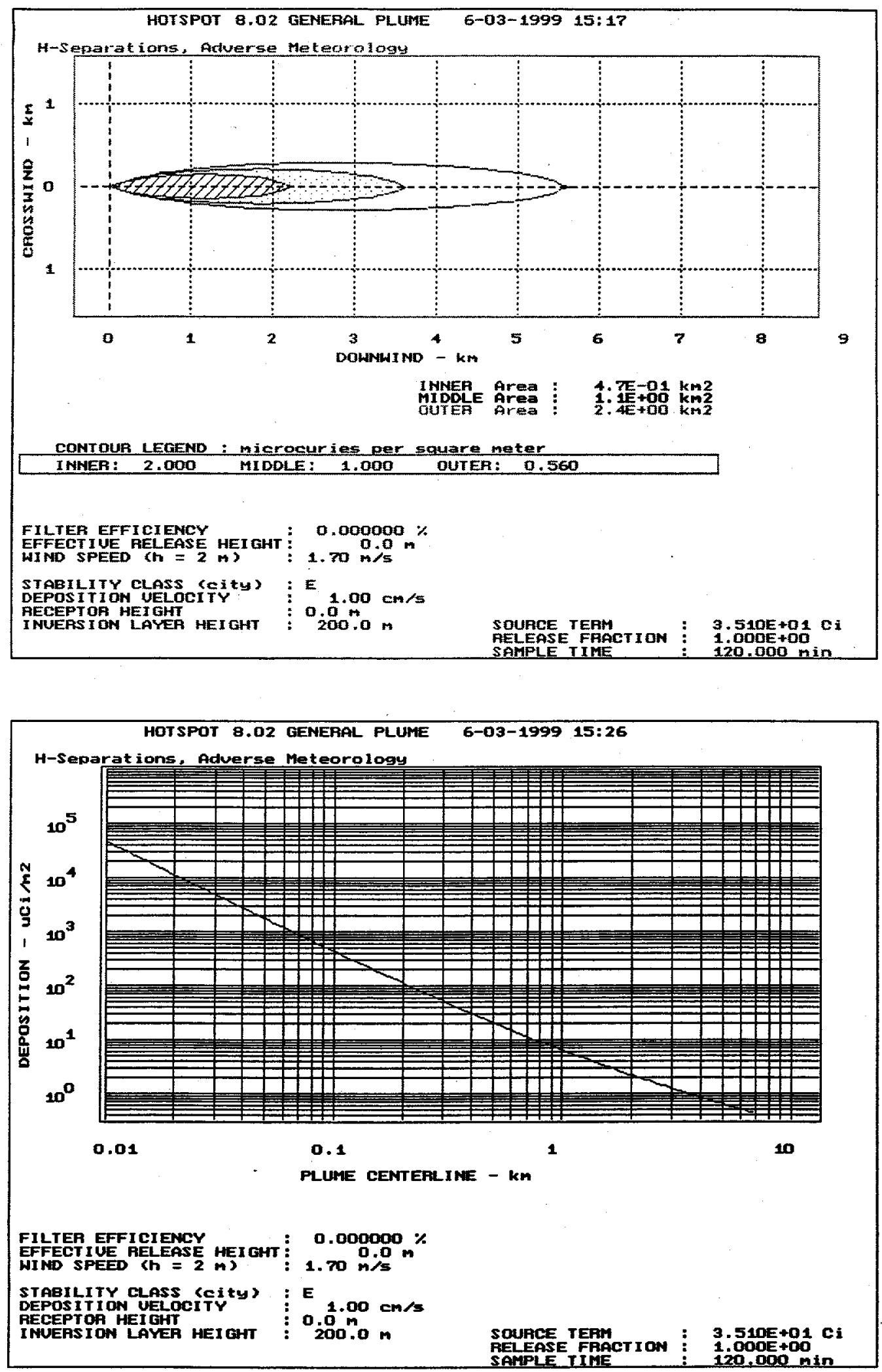


\section{Attachment G}

HOTSPOT results - adverse meteorology, $10 \mathrm{~cm} \mathrm{~s}^{-1}$ 

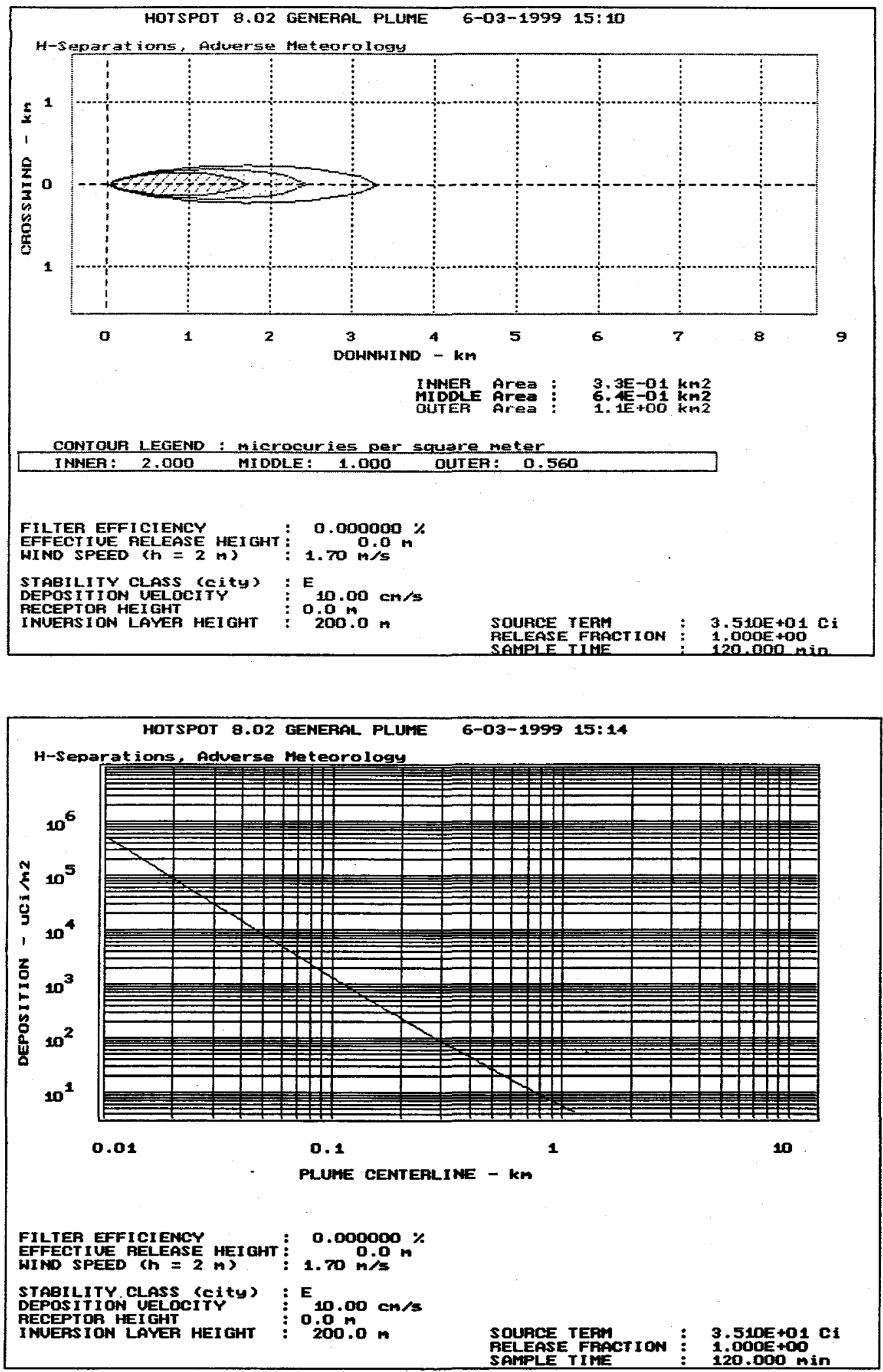